\title{
Biosignal Quality Analysis in Ambulatory Electrocardiograms to Enhance Detection of Myocardial Ischemia
}

by

\section{Patrick Quesnel}

A thesis submitted to the Faculty of Graduate and Postdoctoral Affairs in partial fulfillment of the requirements for the degree of

Master of Applied Science in

Biomedical Engineering

Carleton University Ottawa, Ontario

(C)2014, Patrick Quesnel 


\section{Abstract}

Patients can have an elevated risk of major cardiac complications following non-cardiac surgery. Myocardial ischemia is a lack of oxygen in the heart tissue and may be a precursor to infarction and cardiac death. This ischemia can be detected in the ST segment of a patient's electrocardiogram (ECG) hours before lasting tissue damage occurs. The Perioperative Ischemia Reduction Studies (PROSE) seek to leverage early detection of ischemia to establish an intervention window to stop the ischemic cascade before it results in lasting tissue damage. Ambulatory ECG is used to monitor patients for PROSE; however due to the mobile nature of the recording, ambulatory ECG is subject to higher levels of noise contaminants. This can degrade the ST analysis used in the detection of ischemia, resulting in high false alarm rates that render ambulatory ischemia monitoring clinically impractical. This thesis investigates the possibility of modifying alarm outputs on the basis of ECG signal quality to increase positive predictive value (PPV) of alarms without detriment to existing sensitivity. First, this thesis presents methods to automatically quantify ECG signal quality in a single ECG lead via a signal quality index (SQI) and validates these methods using both non-ischemic and ischemic ECG data. This thesis then proposes and evaluates three system approaches to modifying alarms using this SQI. Resulting modified alarms reveal increased PPV from 0.41 to 0.85 while maintaining sensitivity. These results are encouraging and indicate that these methods could help provide for practical monitoring of myocardial ischemia via ambulatory ECG in the context of PROSE. 


\section{Acknowledgements}

First and foremost I would like to thank my supervisors, Dr. Adrian Chan and Dr. Homer Yang. Their excellent guidance, patience, and encouragement made this the enjoyable experience that it was. I would like to thank my colleagues, Allen Amos-Binks and Geoffrey Clarke for their intellectual support during late nights in the lab and otherwise. I would also like to thank Mohamed Abdelazez for his invaluable technical support in the final months of this thesis.

Finally, I would like to thank my family for their unwavering support. 


\section{Statement of Originality}

This thesis presents the work of the author, under the supervision of Dr. A. D. C. Chan and Dr. H. Yang, completed at Carleton University for the degree of M.A.Sc. in Biomedical Engineering. Many of these results have been presented in conference publications. What follows is a summary of these publications.

P. X. Quesnel, A. D. C. Chan, and H. Yang, "Real-time biosignal quality analysis of ambulatory ECG for detection of myocardial ischemia," in 2013 IEEE International Symposium on Medical Measurements and Applications Proceedings (MeMeA), Gatineau, Canada, 2013, pp. 1-5.

This conference paper describes the signal quality analysis algorithm presented in Chapter 4. The author developed the algorithm using only one of the four metrics examined in Chapter $4\left(S N R_{\min }\right)$ and performed initial validation using non-ischemic ECG data. The manuscript was written, revised following feedback from the co-authors and finalized for publication by the author. This paper was presented as an oral presentation by the author at the 2013 IEEE International Symposium on Medical Measurements and Applications (MeMeA 2013) in Gatineau, Quebec.

P. X. Quesnel, A. D. C. Chan, and H. Yang, "Evaluation of real-time biosignal quality analysis for ambulatory ECG with ST-segment deviations," in 36th Conference of the Canadian Medical \& Biological Engineering Society, Ottawa, Canada, 2013.

This conference paper presents further validation of the signal quality analysis algorithm described in Chapter 4. Validation was performed using both non-ischemic and ischemic 
ECG data and provides the basis for the validation procedure used in Chapter 4. The manuscript was written, revised following feedback from the co-authors and finalized for publication by the author. This paper was presented as an oral presentation by the author at the $36^{\text {th }}$ Conference of the Canadian Medical \& Biological Engineering Society (CMBEC36) in Ottawa, Ontario.

P. X. Quesnel, A. D. C. Chan, and H. Yang, "Signal quality and false myocardial ischemia alarms in ambulatory electrocardiograms," in 2014 IEEE International Symposium on Medical Measurements and Applications (MeMeA), Lisbon, Portugal, 2014, pp. 1-5.

The results of this conference paper appear in Chapter 5. ECG data was contaminated with motion artifact and played through the bedside monitor to acquire ischemia alarms. These were compared to alarms generated from ECG data without contaminations to establish the relationship between ECG signal quality and false ischemia alarms. The manuscript was written, revised following feedback from the co-authors and finalized for publication by the author. This paper was presented as an oral presentation by the author at the 2014 IEEE International Symposium on Medical Measurements and Applications (MeMeA 2014) in Lisbon, Portugal. 


\section{Table of Contents}

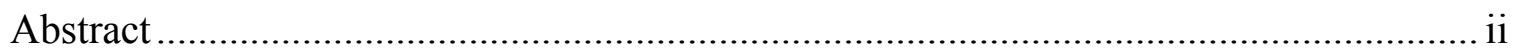

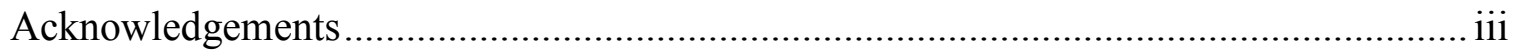

Statement of Originality ..................................................................................... iv

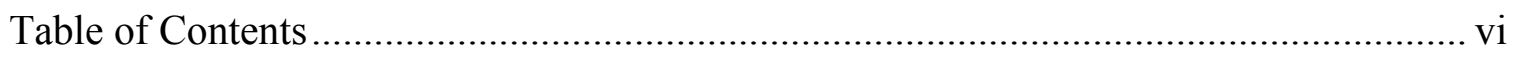

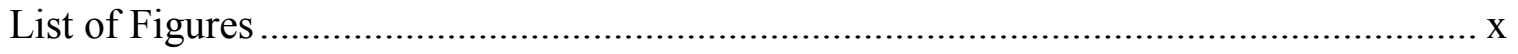

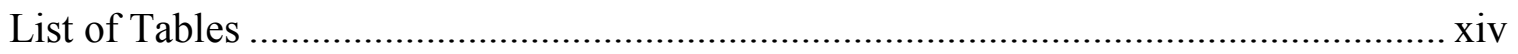

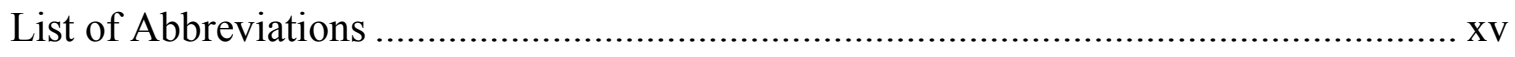

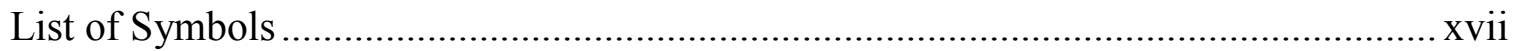

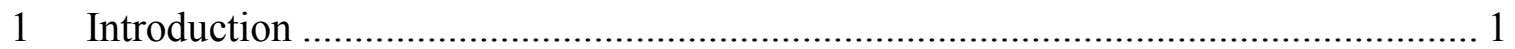

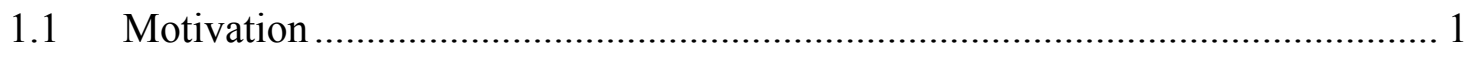

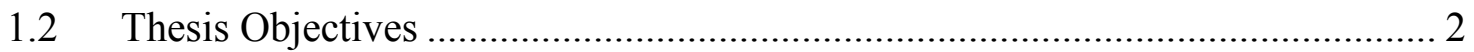

1.3 Summary of Contributions ……………………............................................. 3

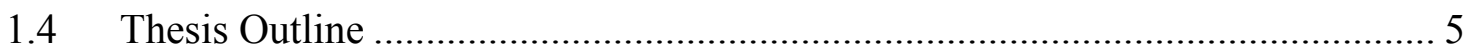

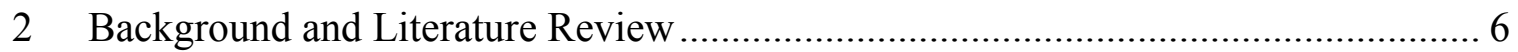

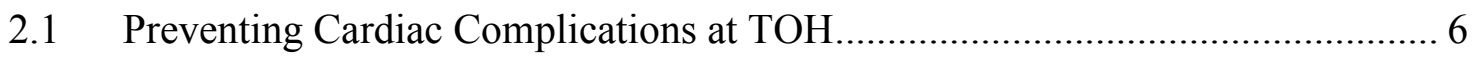

2.1.1 Myocardial Ischemia and Cardiac Complications …………….................... 6

2.1.2 Perioperative Ischemic Evaluation Trial..................................................... 7

2.1.3 Perioperative Ischemia Reduction Studies............................................... 8

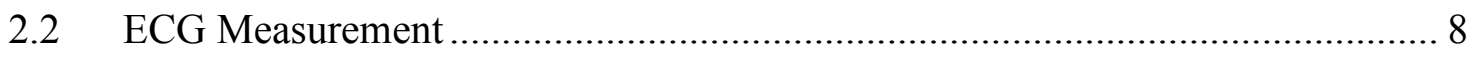

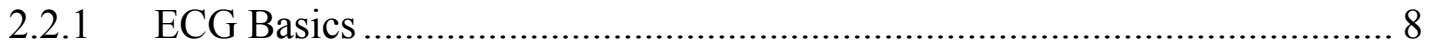

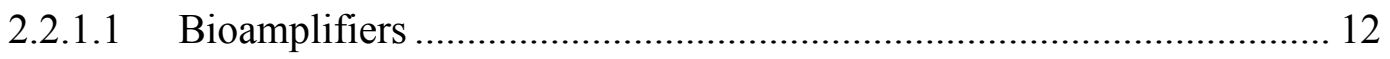

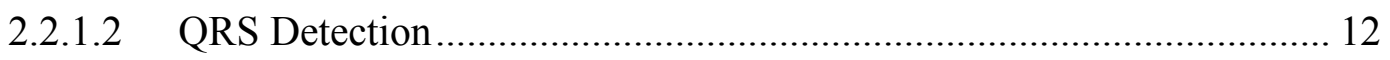

2.2.2 Ambulatory ECG ………………………........................................... 13

2.2.3 ECG Noise Sources............................................................................. 14

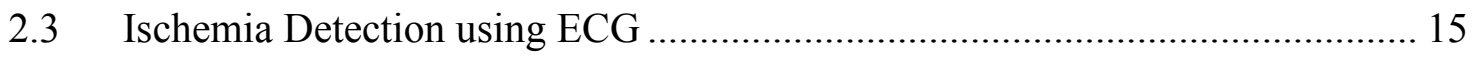

2.3.1 Measuring ST segment deviations ............................................................ 16

2.3.2 Diagnosis of Ischemia ........................................................................... 17

2.3.3 Other causes of ST segment deviations ................................................... 18 


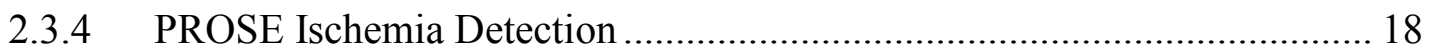

2.3.4.1 PROSE Alarm Criteria and False Alarms ............................................ 19

2.3.4.2 PROSE White Box Filtering ............................................................... 19

$2.4 \quad$ Biosignal Quality Analysis ........................................................................... 21

2.4.1 Rising Need for Biosignal Quality Analysis............................................. 21

2.4.2 Categories of Biosignal Quality Analysis................................................. 22

2.4.3 The State of ECG Biosignal Quality Analysis........................................... 22

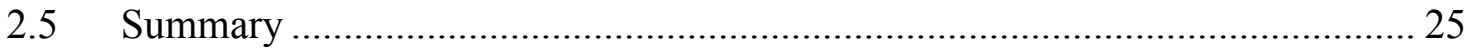

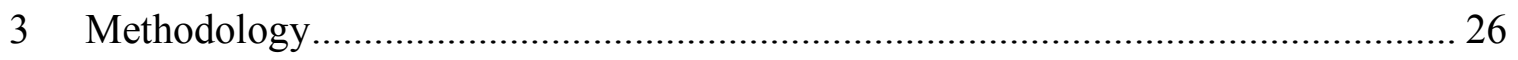

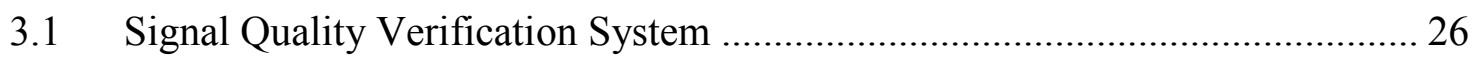

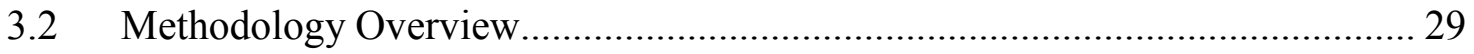

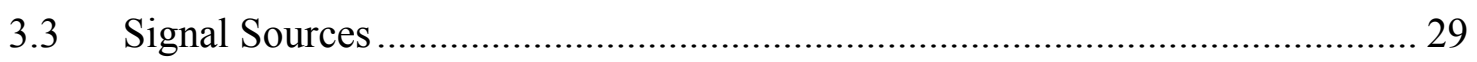

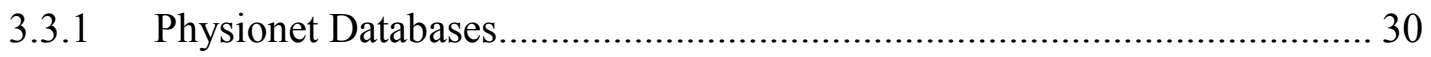

3.3.1.1 Long-Term ST Database ................................................................ 30

3.3.1.2 MIT-BIH Noise Stress Test Database ……………………................. 31

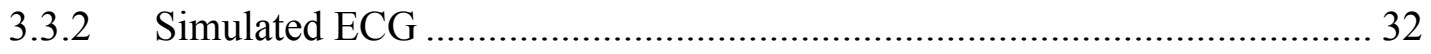

3.4 Equipment Description......................................................................... 33

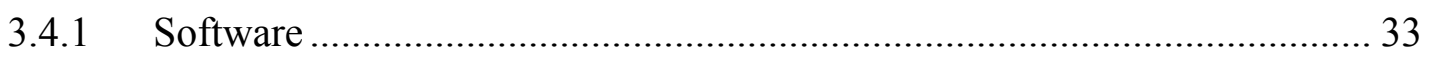

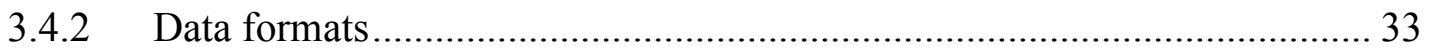

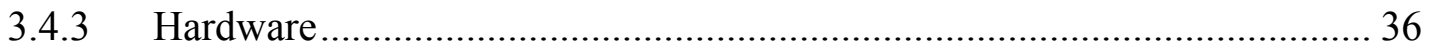

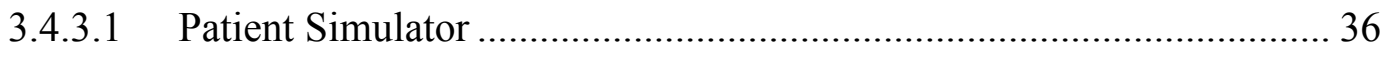

3.4.3.2 Commercial ECG Monitor …………………...................................... 37

3.4.4 Synchronizing Signals .............................................................................. 39

3.4.4.1 Aligning the Start and End of Physionet Data with Logged Bedside

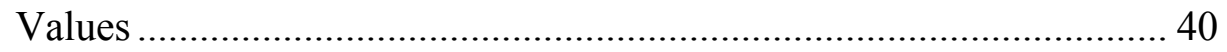

3.4.4.2 Adjusting Logged Timestamps............................................................ 40

3.4.4.3 Repopulating Gaps in Logged Bedside Values ...................................... 41

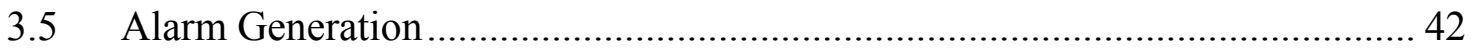

3.5.1 Implemented Alarm Criteria .................................................................. 42

3.5.2 Finite State Machine Implementation ....................................................... 42

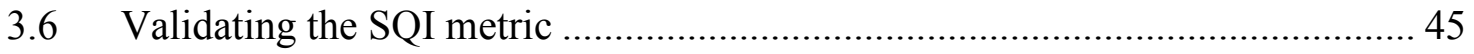


3.7 Investigating Effects of Poor ECG Signal Quality on Alarms......................... 46

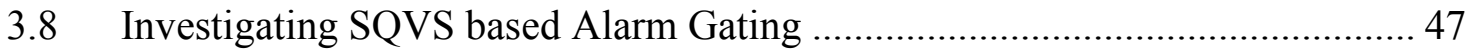

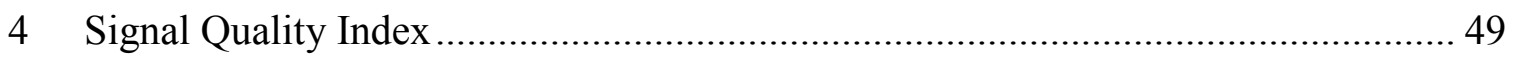

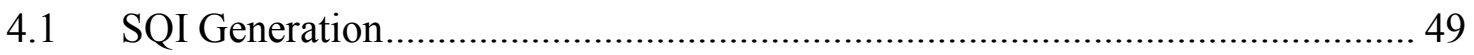

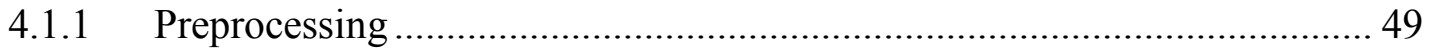

4.1.2 Establishing Analysis Windows .......................................................... 50

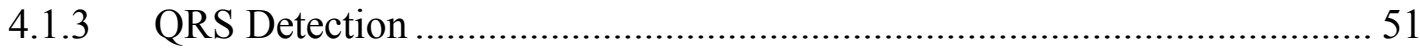

4.1.4 Aligning R Peaks .............................................................................. 51

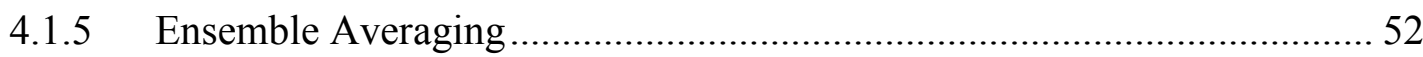

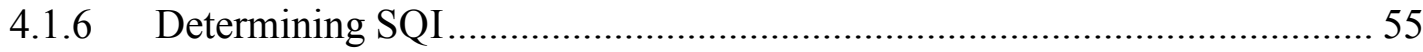

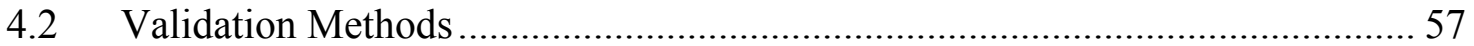

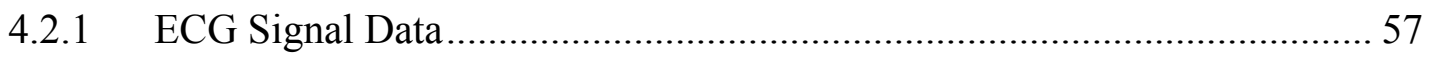

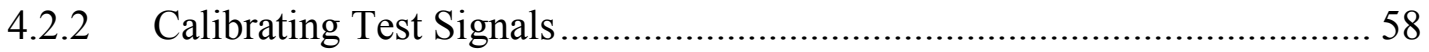

4.2.2.1 Establishing ECG Signal Power ........................................................ 59

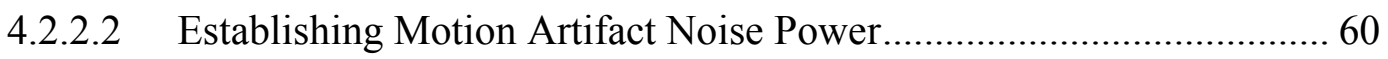

4.2.2.3 Scaling Noise to Calibrate SNR ...................................................... 61

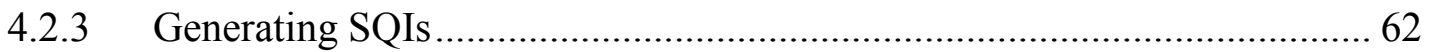

4.2.3.1 Locating and Aligning R Peaks in the Physionet Data....................... 62

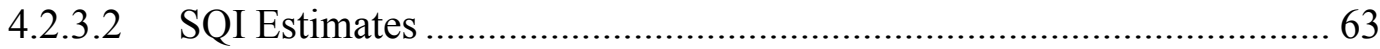

4.2.3.3 Determining Feasibility of Real-Time Deployment.......................... 63

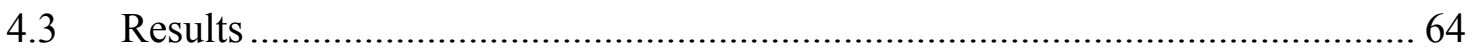

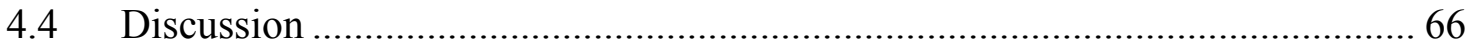

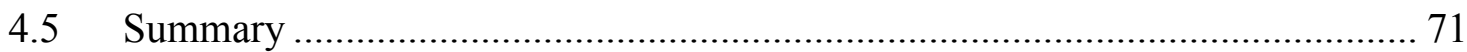

5 Investigating ECG Signal Quality and False Alarms ....................................... 72

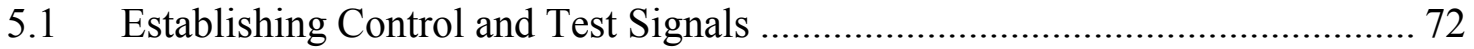

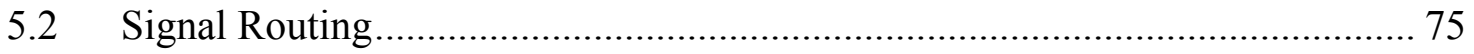

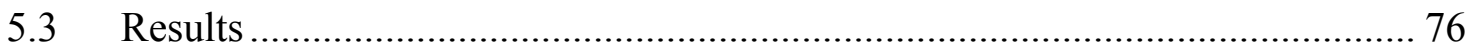

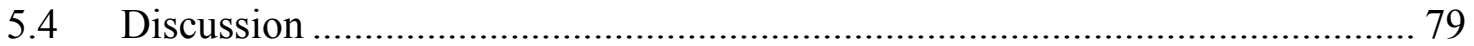

6 Signal Quality Verification System Proof of Concept …................................... 81

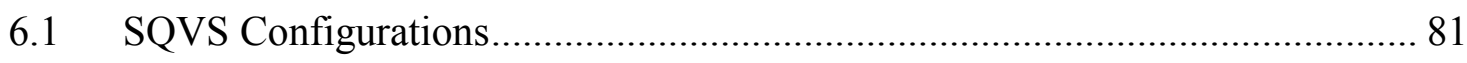




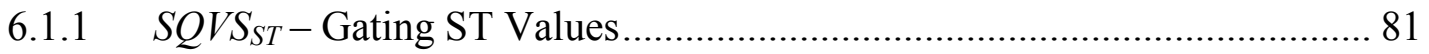

6.1.2 $S Q V S_{E N}-$ Enabling /Disabling the Alarm Generation Stage ...................... 82

6.1.3 SQVS $S_{A L}$ - Gating Generated Alarms ....................................................... 83

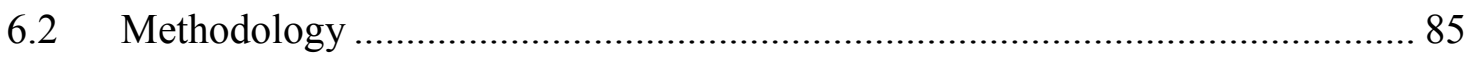

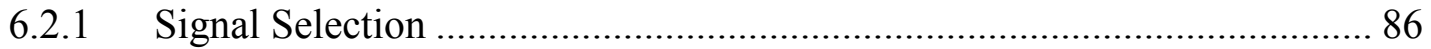

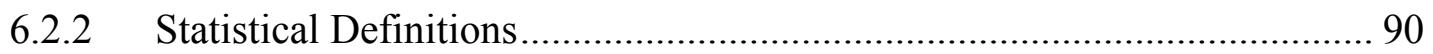

6.2.3 Evaluation Methods ………………………......................................... 93

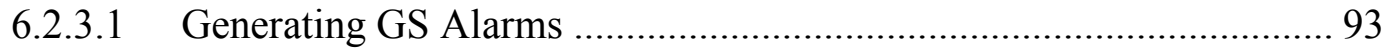

6.2.3.2 Contaminating ECG Records with Motion Artifact............................. 94

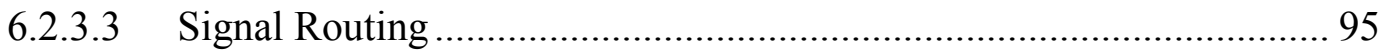

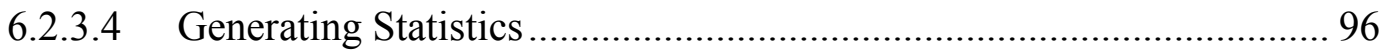

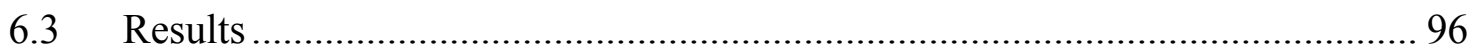

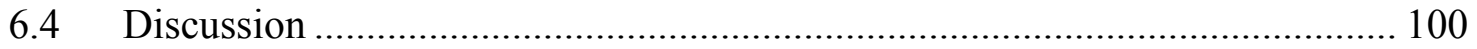

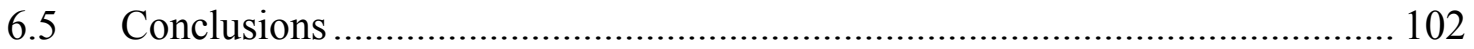

7 Conclusions and Recommendations for Future Work............................................ 103

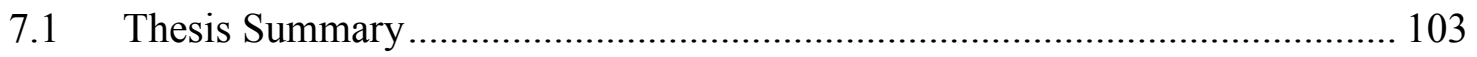

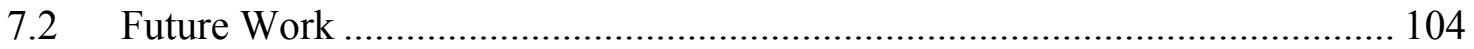

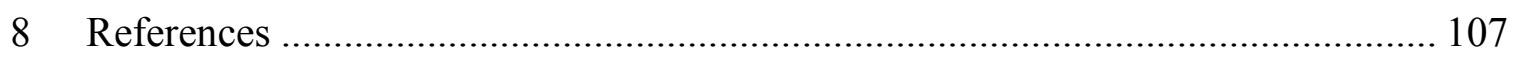

Appendix A: Statistical Counts - First Run........................................................... 114

Appendix B: Statistical Counts - Second Run …………….................................... 116

Appendix C: PPV and Sensitivity for Averaged Counts .............................................. 118 


\section{List of Figures}

Figure 2.1 Anatomy of the human heart showing the four chambers [17] ..................... 9

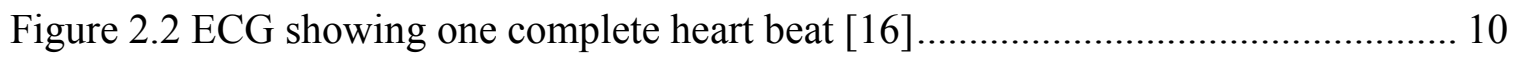

Figure 2.3 Projections of the 12 standard clinical ECG leads [19].............................. 11

Figure 2.4 ECG taken from the patient file 's20021' in the Physionet Long-Term ST Database [38], [39]. (a) normal heart beat (b) beat with an ST segment deviation. 15

Figure 2.5 Signal flow showing original PROSE set up (top) and parallel white box

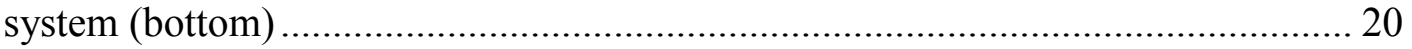

Figure 3.1 Suggested $S Q V S_{S T}$ approach to gate ST values based on poor signal quality

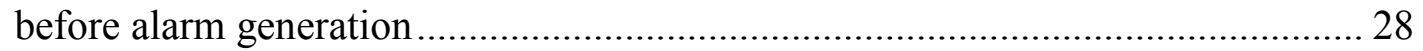

Figure 3.2 Suggested $S Q V S_{E N}$ approach to gate enable/disable the alarm generation...... 28

Figure 3.3 Suggested $S Q V S_{A L}$ approach to gate alarms that are based on poor signal

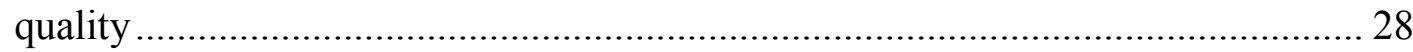

Figure 3.4 Synthetic ECG created using Physionet's 'ecgsyn' simulator ....................... 33

Figure 3.5 Initiating ECG conversion using CONTEC's Case Conversion Software ...... 35

Figure 3.6 Specifying ECG input parameters using CONTEC's Case Conversion

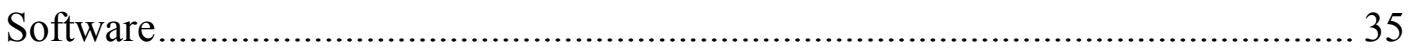

Figure 3.7 MS400 Multiparameter Simulator............................................................. 36

Figure 3.8 Spacelabs Ultraview Digital Telemetry Unit connected to the MS400 patient

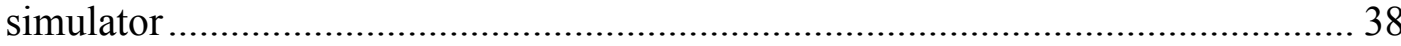

Figure 3.9 Spacelab's Ultraview bedside monitor .................................................... 39 
Figure 3.10 Alarm generating FSM. See Table 3.3 for summary of state transition criteria 45

Figure 3.11 Implemented test setup for investigating the effects of using $S Q V S_{S T}$ to gate ST values. Grayed out portions were not implemented in this thesis 48

Figure 3.12 Implemented test setup for investigating the effects of using $S Q V S_{E N}$ to disable the alarm generating FSM. Grayed out portions were not implemented in this thesis 48

Figure 3.13 Implemented test setup for investigating the effects of using $S Q V S_{A L}$ to gate alarms. Grayed out portions were not implemented in this thesis. 48

Figure 4.1 Zero phase magnitude response of high pass filter used for preprocessing during SQI generation 50

Figure 4.2 Overlaying all beats in a 30 second analysis window to determine the ensemble averaged PQRST template for (a) clean ECG and (b) noisier ECG ....... 54

Figure 4.3 The same ECG data with five levels of calibrated motion artifact noise 62

Figure 4.4 Boxplot showing the relationship between calibrated levels of SNR and four SQI metrics for ECG data without ST deviations ...... 65

Figure 4.5 Boxplot showing the relationship between calibrated levels of SNR and four SQI metrics for ECG data with ST deviations 65

Figure 4.6 Ensemble averaging 30 seconds of ECG with periodic motion artifact that matches the HR in a segment calibrated to have a $-10 \mathrm{~dB}$ SNR...... 68

Figure 4.7 Ensemble averaging the 30 second segment immediately before the one shown in Figure 4.5. 69 
Figure 4.8 Ensemble averaging a contaminant-free 30 second segment containing a PVC

Figure 5.1 Transition from clean to contaminated ECG in the test signal used to investigate the relationship between ECG signal quality and false alarms 74

Figure 5.2 Signal flow depicting ST value generation and parallel SQI generation for testing the relationship between ECG signal quality and false alarms. 76

Figure 5.3 (a) ST values and (b) SQIs for the control signal. Red dotted lines indicate ischemia alarm boundaries 77

Figure 5.4 (a) ST values and (b) SQIs for the test signal. Hatched sections indicate the occurrence and duration of myocardial ischemia alarms 78

Figure 6.1 Myocardial ischemia alarm signal flow, highlight components of the $S Q V S_{S T}$ used to gate ST values before they reach the alarm generation stage 82

Figure 6.2 Myocardial ischemia alarm signal flow, highlighting components of the $S Q V S_{E N}$, which disables the alarm generating FSM during periods of poor signal quality 83

Figure 6.3 Myocardial ischemia alarm signal flow, highlighting components of $S Q V S_{A L}$ used to gate the alarm output of the alarm generating FSM. 84

Figure 6.4 Varying responses from the three SQVS configurations to the same set of SQI and ST value inputs. Hatched sections indicate an alarm output was allowed out of the system and gray dotted line indicates SQI threshold. 85

Figure 6.5 Lead II ST values from full-length 's20121' record resulting in full-length alarm. Hatched area indicates that an alarm is active. 87 
Figure 6.6 Bedside monitor showing history of recent ST values. Note how the marker indicating the ST measurement site remains steady while the marker indicating the

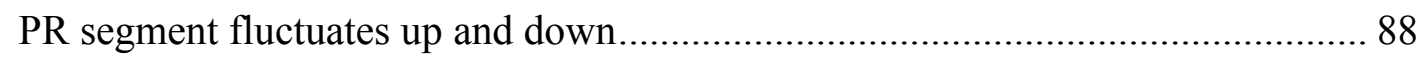

Figure 6.7 Lead II ST values from full-length s20051 record resulting in three distinct ischemia alarms. Hatched areas indicate an alarm is active .................................... 89

Figure 6.8 Simulated alarms and gold standard ischemic episodes to demonstrate definition of TP

Figure 6.9 Simulated alarms and gold standard ischemic episodes to demonstrate the

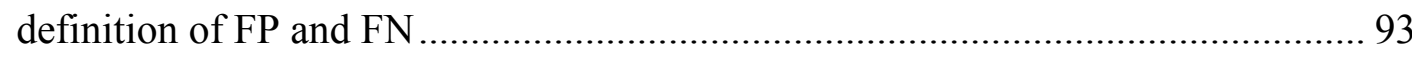

Figure 6.10 TP, FP, and FN counts for each SQVS configuration plotted against the SQI threshold at which they were attained - averaged across two runs......................... 97

Figure 6.11 Precision Recall Curve showing PPV and sensitivity calculated from alarm counts that were averaged across two runs for three SVQS configurations using various SQI thresholds 98 


\section{List of Tables}

Table 3.1 Physionet's Long-Term ST Database records with Lead II and V2 data......... 31

Table 3.2 Summary of PROSE alarm criteria ..................................................... 42

Table 3.3 Summary of transition conditions for the alarm generating FSM .................. 44

Table 4.1 High pass filter coefficients for pre-processing ECG data prior to SQI

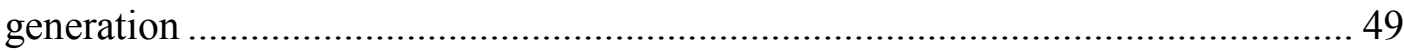

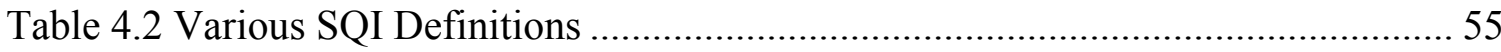

Table 4.3 Begining, end, and maximal deviation measurements and their elapsed times times for a 30 minute iscehmic episode in record ' 20031 '................................... 58

Table 4.4 Correlation coefficients for the validation of four metrics used in the SQI

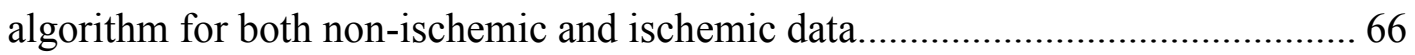

Table 5.1 Power of ECG and motion artifact components used for testing the relationship between ECG signal quality and false alarms ................................................ 75

Table 6.1 Number and type of alarms occurring in records used to evaluate SQVS configurations 90

Table 6.2 Highest attained PPV without loss of sensitivity for each SQVS configuration 99

Table 6.3 Highest retained sensitivity to achieve 100\% PPV for each SQVS configuration 


\section{List of Abbreviations}

$\begin{array}{ll}\text { ADC } & \text { Analog to Digital Converter } \\ \text { BIH } & \text { Beth Israel Hospital } \\ \text { BPM } & \text { Beats per minute } \\ \text { CMRR } & \text { Common Mode Rejection Ratio } \\ \text { ECG } & \text { Electrocardiogram } \\ \text { ECRI } & \text { Emergency Care Research Institute }\end{array}$

FN False Negative

FP False Positive

FSM Finite State Machine

GS Gold Standard

HR Heart Rate

KLT Karhunen-Loève Transform

LOS Length of Stay

MIT Massachusetts Institute of Technology

NBIB National Institute of Biomedical Imaging \& Bioengineering

NIGMS National Institute of General Medical Sciences 


$\begin{array}{ll}\text { NIH } & \text { National Institutes of Health } \\ \text { PC } & \text { Personal Computer } \\ \text { POISE } & \text { Perioperative Ischemic Evaluation } \\ \text { PPV } & \text { Positive Predictive Value } \\ \text { PRC } & \text { Precision Recall Curve } \\ \text { PROSE } & \text { Perioperative Ischemia Reduction Studies } \\ \text { SQI } & \text { Signal Quality Index } \\ \text { SQVS } & \text { Signal Quality Verification System } \\ \text { SVM } & \text { Support Vector Machine } \\ \text { TOH } & \text { The Ottawa Hospital } \\ \text { TP } & \text { True Positive }\end{array}$




\section{List of Symbols}

$\mathrm{A}_{\mathrm{cm}} \quad$ Common mode gain of a bioinstrumentation amplifier

$\mathrm{A}_{\mathrm{d}} \quad$ Differential gain of a bioinstrumentation amplifier

$B_{i} \quad$ Baseline (average) value of Noise $_{i}$

$C_{k} \quad$ Scaling factor for calibrating SNR

Err $r_{\text {corr }} \quad$ R peak alignment error correction factor

M The total number of heart beats in an SQI analysis window

Noise $_{\text {avg, } R M S} \quad$ Mean $R M S_{i}$ value during a 30 second SNR calibration segment

Noise $_{i} \quad$ The $i^{\text {th }}$ second of motion artifact used during SNR calibration

Noise $_{m, r m s} \quad$ RMS value of the noise present on the $m^{\text {th }}$ heart beat

$P_{E C G} \quad$ Signal power of the ECG of a 30 second SNR calibration segment

$P_{\text {Noise }} \quad$ Signal power of motion artifact noise during a SNR calibration segment

$Q R S_{p k p k, a v g} \quad$ Average peak to peak amplitude of the QRS complexes

$r \quad$ Pearson correlation coefficient

$R M S_{i} \quad$ RMS value of the differences between Noise $e_{i}$ and $B_{i}$

$S N R_{m} \quad$ SNR for the $m^{\text {th }}$ heart beat

$\mathrm{SQI}_{25} \quad 25^{\text {th }}$ percentile SNR within an SQI analysis window 
$S Q I_{\text {mean }}$

$S Q I_{\text {median }}$

$S Q I_{\min }$

$S Q V S_{A L}$

$S Q V S_{E N}$

$S Q V S_{S T}$

$W_{\text {avg }}$

$W_{\text {avg,rms }}$

$W_{m}$

$\mathrm{V}_{+}$

V.

$\mathrm{V}_{\mathrm{o}}$
Mean SNR within an SQI analysis window

Median SNR within an SQI analysis window

Minimum SNR within an SQI analysis window

Signal Quality Verification System based on gating alarm output

Signal Quality Verification System based on enabling the alarm FSM

Signal Quality Verification System based on gating ST values

The average PQRST waveform in an SQI analysis window

RMS value of the average PQRST waveform

The $m^{\text {th }}$ complete heart beat in an SQI analysis window

Voltage at the positive electrode

Voltage at the negative electrode

Output voltage from a bioinstrumentation amplifier 


\section{Introduction}

\subsection{Motivation}

Patients that undergo non-cardiac surgery have the potential to experience major cardiac complications such as myocardial infarctions, cardiac arrests, and cardiac death [1]. In fact, as many as 900,000 patients globally experience a major cardiac event following non-cardiac surgery each year [2] and with the aging cohort, an increasing number of patients are undergoing non-cardiac surgery. These complications result in, at a minimum, an increase in patient length of stay (LOS) and consequently an increase in the cost of health care provision [2]. A significant cause of these major cardiac complications is due to a decrease in blood flow to the heart - a condition known as myocardial ischemia [1].

Myocardial ischemia is detectable via slow changes in a patient's electrocardiogram (ECG) [3], which is the electrical signal generated by the heart's muscle activity. Detection of myocardial ischemia allows for an intervention window to stop the ischemic cascade before it results in myocardial infarction [4]. Typical ECG monitoring employs a stationary bedside monitor, which is connected to the patient via a series of wires and thus limits patient mobility. In contrast, ambulatory ECG employs a portable device to record the patient's ECG data, which can then be wirelessly transmitted for real-time analysis. Unfortunately, issues with signal quality that are associated with ambulatory ECG result in frequent false alarms when ambulatory ECG is used for the detection of myocardial ischemia [5]. 
Given the number of medical alarms facing healthcare providers each day, false alarms actually present a growing hazard. Significant numbers of alarms alerting healthcare providers are of low clinical significance. This low significance is synonymous with low alarm positive predictive value (PPV) and leads to alarm desensitization (also called alarm fatigue) [6]. Alarm fatigue has appeared on the Emergency Care Research Institute's (ECRI) list of top ten health technology hazards since 2007 - frequently topping that list, including most recently in 2014 [7].

The globally increasing number of non-cardiac surgeries affiliated with the aging cohort and the dangers presented by alarm fatigue both highlight the need for improved detection of myocardial ischemia. This thesis presents a myocardial alarm gating system that is based on the ECG signal quality. The presented system automatically assesses signal quality and establishes a Signal Quality Index (SQI). This SQI is used to gate alarms such that the PPV of myocardial ischemia alarms is increased without detriment to the alarm sensitivity.

\subsection{Thesis Objectives}

The overall objective of this thesis is to develop a method, based on biosignal quality analysis, to improve the PPV of myocardial ischemia detection in ambulatory ECG, without adversely affecting its sensitivity. This thesis specifically presents the following objectives:

1. Develop and evaluate an algorithm that automatically assess ECG signal quality and provides a representative SQI. 
2. Investigate potential of the SQI to reduce false alarms while maintaining current sensitivity levels.

\subsection{Summary of Contributions}

This thesis presents the following major contributions:

\section{Development of an ECG signal quality analysis algorithm}

Periods of poor ECG signal quality can result in false ischemia alarms. Thus, being able to identify periods of more signal quality may help to identify false alarms as they occur. This thesis describes an ECG signal quality analysis algorithm that leverages the repeatability of the PQRST complex generated by a patient's heartbeat. The algorithm generates an SQI based solely on one single ECG lead, thus not requiring any information from additional leads or sensors. This results in a light, portable algorithm that can be used with any collected ECG data. While this thesis focuses on improving myocardial ischemia alarms, the SQI may be applicable in other areas employing ECG such as ECG biometrics, fitness or other clinical applications.

\section{Performance evaluation of the aforementioned algorithm using ECG from the} target group; patients with and without ST segment deviations

Myocardial ischemia is detected via changes in a patient's ECG. Since the ECG signal quality analysis algorithm is based on the repeatability of a patient's ECG, it was necessary to evaluate the algorithm using ECG data containing these ischemic changes. To accomplish this, ECG was contaminated with known quantities of noise 
and SQIs were generated for these contaminated signals. The correlations between the SQI and the known level of contamination were compared for both ECG with and without ST deviations. Results showed that the algorithm performed comparably for both normal ECG data and ECG data reflecting myocardial ischemia, with Pearson correlation coefficients as high as 0.96 calculated for both ST-deviated and non-ST deviated ECG. This indicates that the algorithm is suitable for use on ischemic ECG data.

\section{Demonstrated that gating alarms on the basis of signal quality has the ability to} reduce false positives generated from periods of poor signal quality

To investigate the effects of modifying alarms on the basis of signal quality ambulatory ECGs containing myocardial ischemic episodes were artificially contaminated with motion artifact. ST deviations for these contaminated ECG data were recorded by a bedside monitor and were analyzed twice for alarms, once with alarm modification based on the SQI and once without. The alarm gating system effectively improved PPV of alarms from 0.41 to 0.85 while still preserving the sensitivity of the system. These results are encouraging and indicate that these methods could help provide for practical monitoring of myocardial ischemia via ambulatory ECG in the context of the Perioperative Ischemia Reduction Studies (PROSE) taking place at The Ottawa Hospital (TOH).

Portions of this research have been disseminated in the following papers:

- P. X. Quesnel, A. D. C. Chan, and H. Yang, "Real-time biosignal quality analysis of ambulatory ECG for detection of myocardial ischemia," in 2013 IEEE International 
Symposium on Medical Measurements and Applications Proceedings (MeMeA), Gatineau, Canada, 2013, pp. 1-5.

- P. X. Quesnel, A. D. C. Chan, and H. Yang, "Evaluation of real-time biosignal quality analysis for ambulatory ECG with ST-segment deviations," in 36th Conference of the Canadian Medical \& Biological Engineering Society, Ottawa, Canada, 2013.

- P. X. Quesnel, A. D. C. Chan, and H. Yang, "Signal quality and false myocardial ischemia alarms in ambulatory electrocardiograms," in 2014 IEEE International Symposium on Medical Measurements and Applications (MeMeA), Lisbon, Portugal, 2014, pp. 1-5.

\subsection{Thesis Outline}

The remaining chapters of this thesis are structured as follows. Chapter 2 describes background information on myocardial ischemia and studies taking place at $\mathrm{TOH}$ aimed at reducing the occurrence of major cardiac complications following non-cardiac surgery. Chapter 2 also contains a high-level review of literature related to biosignal quality analysis. Chapter 3 outlines the methodology employed while performing the research for this thesis; this includes descriptions of equipment and ECG databases used. Chapter 4 presents the SQI algorithm, along with validation of the SQI. Chapter 5 examines the relationship between signal quality and false alarms. Chapter 6 discusses proof-ofconcept testing of the alarm gating system, specifically targeting increased PPV while maintaining sensitivity. Finally, Chapter 7 summarizes this thesis and presents recommendations for future work. 


\section{Background and Literature Review}

In order to understand the context of this false alarm reduction research project, this chapter discusses on-going efforts at $\mathrm{TOH}$ to reduce the occurrence of major cardiac complications following non-cardiac surgery. Then, the subsequent sections examine the relevant literature on ECG, its role in the detection of myocardial ischemia, and biosignal quality analysis. The aim of this section is to paint a context from which the reader can understand the contributions presented in this thesis and the rationale behind the suggested approach.

\subsection{Preventing Cardiac Complications at $\mathrm{TOH}$}

\subsubsection{Myocardial Ischemia and Cardiac Complications}

The trauma, pain, anesthesia, fasting, bleeding, anemia, and hypothermia associated with surgery act as an extreme stress test on the body [2]. These stresses can ultimately cause decreased blood flow to the heart muscle, also called myocardial tissue, while simultaneously increasing oxygen demand, resulting in myocardial ischemia [2]. Ischemia is a condition whereby blood flow is restricted to an area of the body, resulting in a shortage of oxygen and nutrients required for proper cell function. Myocardial ischemia is strongly associated with myocardial infarction [8], [9]. Infarction refers to tissue death due to a lack of oxygen; myocardial infarction specifically refers to death of a portion of the heart tissue. 
With the aging cohort, a growing number of patients undergo non-cardiac surgery each year [10]. Around the world, as many as 900,000 of these patients experience a major cardiac event such as cardiac death, nonfatal myocardial infarction, or cardiac arrest as a result of their surgery [2]. These complications result in, at minimum, an increase in patient LOS by an average of 7 days [11]. It has been estimated that a major complication can result in hospital cost increases of more than $\$ 11,000$ per complication [12]. With today's laparoscopic surgery techniques, surgeries are becoming less invasive. When coupled with improvements in surgical pain management, this can allow for earlier patient discharge. When possible, early discharge often proves better for the patient (e.g., better atmosphere, reduced risk of hospital-acquired infections [13], etc.) and helps to reduce hospital operating costs [14]. However, early discharge is not an option for patients deemed at risk for complications (risk, in the context of major cardiac events, is discussed in the following section). Administration of $\beta$-blockers has shown potential to mitigate this chance of complication; this was investigated by the Perioperative Ischemic Evaluation (POISE) trial and, subsequently, PROSE as described in the following sections.

\subsubsection{Perioperative Ischemic Evaluation Trial}

Patients at risk of experiencing a major cardiac complication can be identified prior to surgery via a set of risk factors; such as history of ischemic heart disease, use of insulin pump for diabetes, etc. [15]. Cardiovascular complications account for up to $60 \%$ of postoperative mortalities in patients deemed 'moderate' to 'high' risk [1]. POISE sought to assess the effectiveness of prophylactic administration of $\beta$-blockers to all patients identified as having an elevated risk of complication [1]. They found that $\beta$-blocker 
regimens decreased the occurrence of myocardial infarction but caused increased incidence of bradycardia, hypotension, and stroke [1].

\subsubsection{Perioperative Ischemia Reduction Studies}

The findings of POISE lead to the establishment of PROSE. While POISE explored the blanket approach of prophylactic $\beta$-blocker administration to all at-risk patients, the ongoing PROSE seeks to investigate selective administration of $\beta$-blockers only to patients experiencing myocardial ischemia [5]. Specifically, PROSE is evaluating the possibility of early detection of an ischemic episode to allow for an intervention window to stop the ischemic cascade before it results in myocardial infarction [4], [5]. This approach only targets patients that would benefit from $\beta$-blocker administration while avoiding patients who do not need it. This selective approach should avoid the complications found in POISE. PROSE uses ambulatory ECG (described in Section 2.2.2) to detect ischemic episodes. The term ambulatory refers to the ability of a patient to be ambulating (e.g., mobile) while being monitored. Such monitoring, when coupled with telemedicine (discussed in Section 2.4.1) could ultimately allow home monitoring for myocardial ischemia thereby enabling early discharge of at-risk patients.

\subsection{ECG Measurement}

\subsubsection{ECG Basics}

Aside from the stethoscope, ECG is the oldest tool available to cardiologists [16]. It is a recording of the electrical activity associated with a patient's heart. The human heart consists of four distinct chambers; two atria and two ventricles, as illustrated in Figure 
2.1. The left and right atria pump blood into the left and right ventricles, respectively. The right and left ventricles pump blood out of the heart to the lungs and body, respectively.

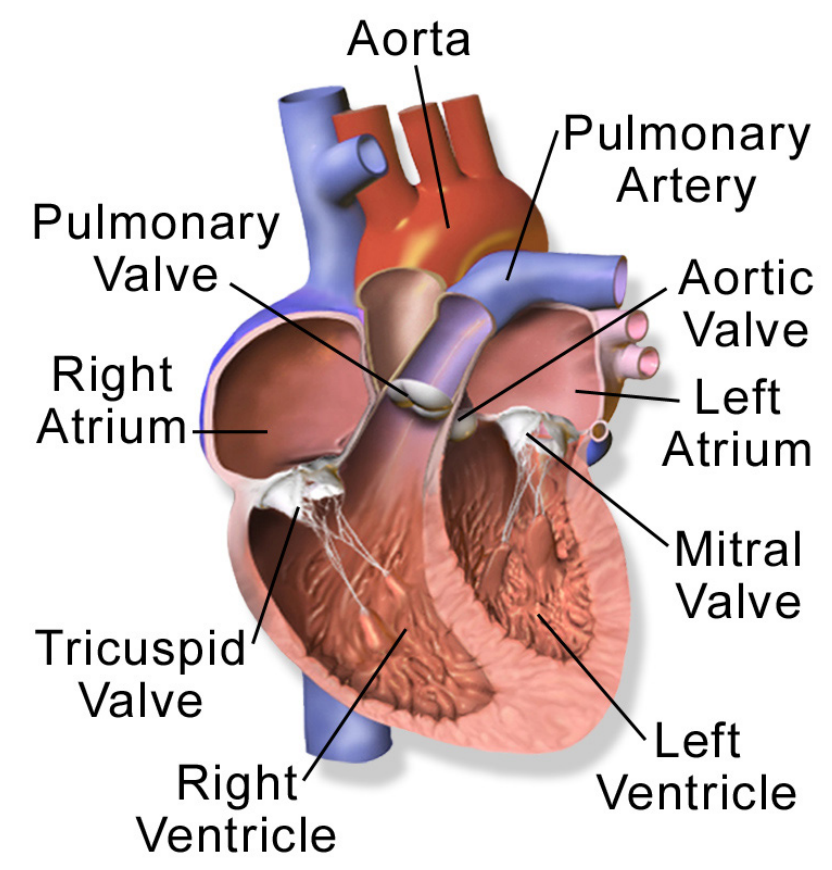

Figure 2.1 Anatomy of the human heart showing the four chambers [17]

The electrochemical changes responsible for contraction of the various chambers of the heart result in voltage changes on a patient's ECG. One complete heart beat renders the PQRST complex illustrated in Figure 2.2. The $\mathrm{P}$ wave is due to activation of the atrial muscles, the QRS complex is the result of both repolarization of the atria and activation of the ventricles, and the $\mathrm{T}$ wave is due to repolarization of the ventricles. The strength and polarity of these P, QRS, and T waves depend on the ECG measurement sites [18], (i.e., electrode locations). 


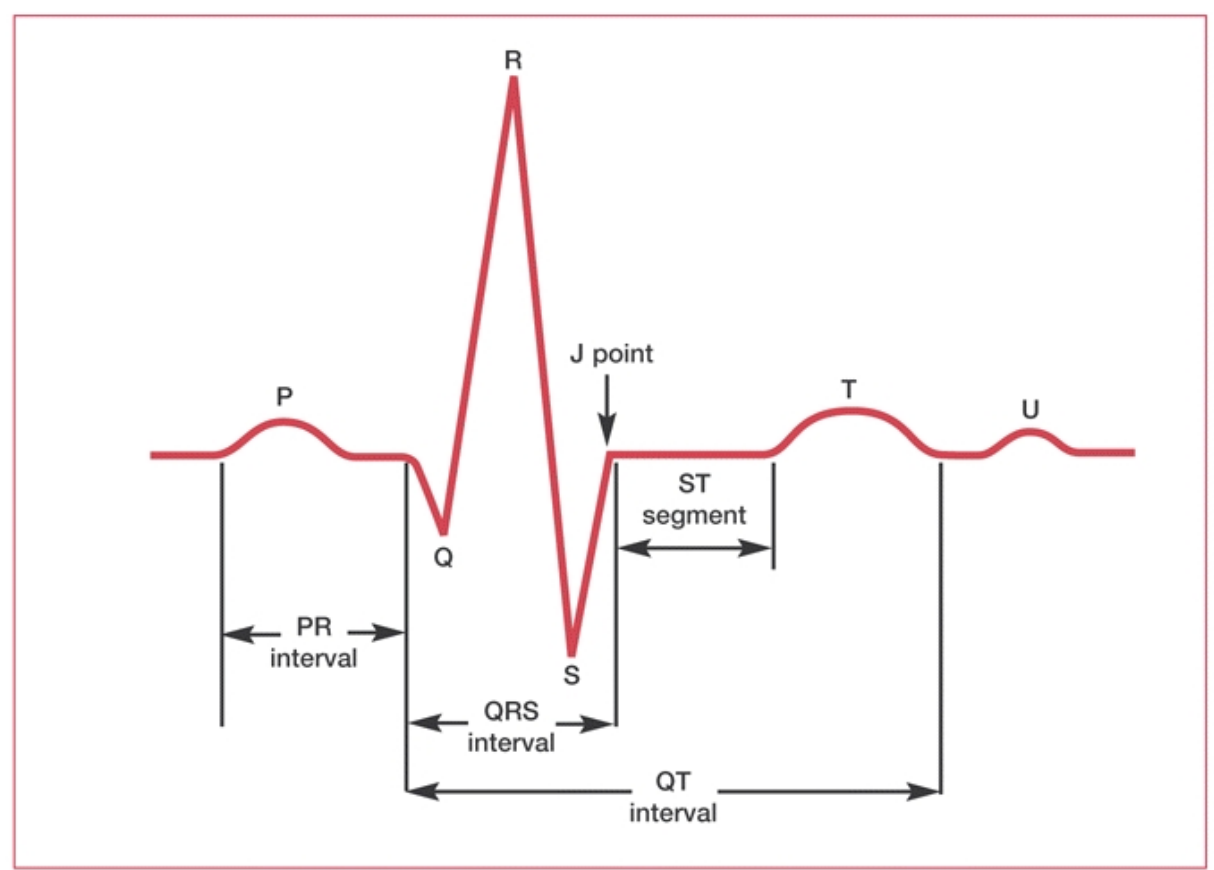

Figure 2.2 ECG showing one complete heart beat [16]

The orientation between two electrodes is called a lead. Standard clinical ECG uses 12 leads. Leads I, II and III are bipolar limb leads employing dedicated electrodes on the left arm, right arm, and left leg. V1 - V6 are unipolar precordial leads, each employing one dedicated electrode on the chest and all sharing the average input from Leads I, II, and III (called Wilson's central terminal). Lastly, aVR, aVL and aVF are augmented leads, respectively using the electrode on the right arm, left arm or left foot as a positive electrode and deriving the negative electrode from the remaining two limb electrodes [19]. Each lead provides a different view of the heart. Note that the different leads represent electrical projections of the heart in different planes of the body, as shown in Figure 2.3; the sagittal plane divides the body into left and right, transverse divides the body into top and bottom, and frontal divides the body into front and back. 


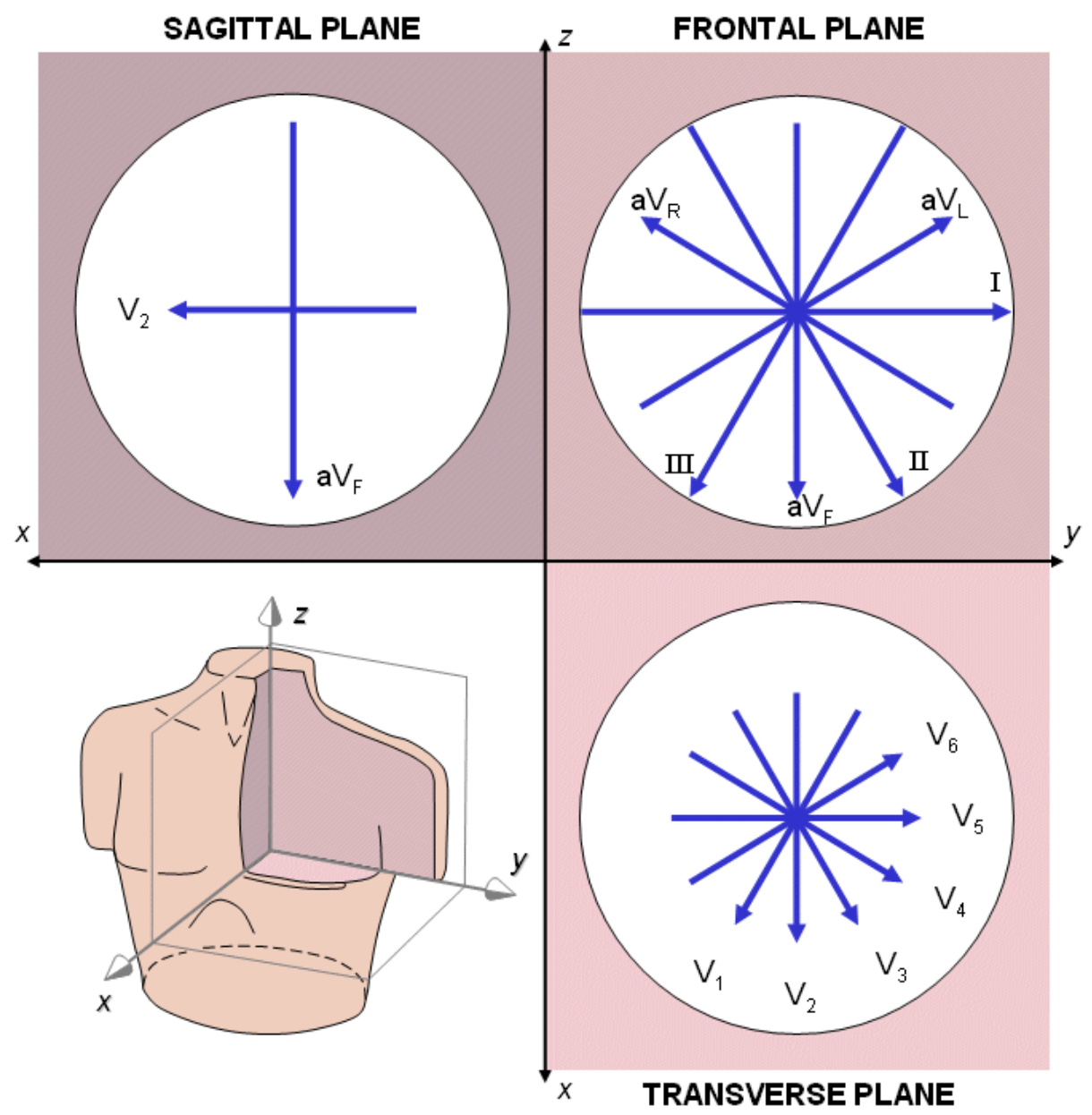

Figure 2.3 Projections of the 12 standard clinical ECG leads [19]

The electric fields generated by contractions of the myocardial tissue must pass through tissue in the body to reach the electrodes, which are located on the surface of the skin. This introduces volume-conductor effects, which attenuate and low-pass filter the electric fields [18]. By the time they are measured on the surface of the skin, ECG peak voltage is typically on the order of $1-5 \mathrm{mV}$ [18]. Significant portions of ECG data is contained in the bandwidth between $1-40 \mathrm{~Hz}$; however clinically relevant information also exists outside this range. Consequently, currently recommended practice is to acquire diagnostic ECG in the bandwidth of $0.67-250 \mathrm{~Hz}$ using zero phase shift filters [20] . 


\subsubsection{Bioamplifiers}

To acquire such small signals, specialized bioamplifiers are used. These bioamplifiers function by amplifying the difference between electrodes while rejecting any signal that is common to both electrodes [18]. The voltage output of a bioamplifier can be written as

$$
V_{o}=A_{d}\left(V_{+}-V_{-}\right)+A_{c m}\left(\frac{V_{+}+V_{-}}{2}\right)
$$

where $V_{o}$ is the voltage output, $A_{d}$ is the differential gain, $A_{c m}$ is the common mode gain, and $V_{+}$and $V_{-}$are the voltages on the positive and negative electrodes, respectively. The differences between $V_{+}$and $V_{\text {- }}$ are amplified by the differential gain, while the voltage common to both $V_{+}$and $V_{\text {- }}$ is amplified by the common mode gain. Bioamplifiers are typically characterized by their common mode rejection ration (CMRR) which is the ratio, in $\mathrm{dB}$, between $A_{d}$ and $A_{c m}$ as illustrated in (2.2).

$$
C M R R=10 \times \log _{10}\left(\frac{A_{d}}{\left|A_{c m}\right|}\right)^{2}
$$

In this way, the electrical activity of the heart is isolated from much larger signals occurring across the whole body, such as capacitive coupling to electrical power lines.

\subsubsection{QRS Detection}

The QRS complex, a distinguishable feature of the PQRST complex, is typically used to denote the occurrence of a heart beat. Knowing the location of QRS complexes within a subject's ECG provides many importance insights into that subject's health. The most obvious of these insights is heart rate (HR), but this can also extend to more obscure insights, such as a subject's emotional state via monitoring variations in the HR [21]. Automatic detection of QRS complexes is mandatory to make these insights a feasible 
reality. Consequently, various QRS detection methods have been developed that can robustly deal with signal noise (noise sources are discussed in Section 2.2.3). Pan and Tompkins [22] developed a multistep algorithm to amplify the QRS structure allowing real time detection; the Pan-Tompkins algorithm is an established and accurate method for QRS detection. Since then QRS detection has assumed many forms, with some of the more recent ones using empirical mode decomposition [23] and wavelet transforms, achieving sensitivity of $99.64 \%$ and PPV of $99.82 \%$ [24].

\subsubsection{Ambulatory ECG}

Typical clinical ECG monitoring employs a fixed bedside monitor with wires (also called leads but these physical leads/wires should not to be confused with the measurement leads as discussed in Section 2.2.1) connecting it to the electrodes on the patient. This setup physically limits patient movement and confines them to their bed while being monitored. Ambulatory ECG, on the other hand, employs a portable device to record the patient ECG data. Various configurations for ambulatory ECG exist. For example, Holter monitors record the ECG data to onboard storage for later offline analysis. Alternatively, ambulatory ECG devices can also wirelessly transmit ECG data for remote storage, or even for remote real-time analysis. In this thesis, ambulatory ECG is considered to be wirelessly transmitted for real-time analysis. These ambulatory ECG configurations are typically used to acquire long-term records for observing intermittent symptoms that may otherwise be difficult to capture when the patient is physically confined by typical bedside monitors [25]. 
To monitor for myocardial ischemia, PROSE uses ambulatory ECG (with remote, realtime analysis). Following non-cardiac surgery, patients are typically ambulating, necessitating ECG monitoring to also be ambulatory. Unfortunately, patient movement subjects the ECG to added motion artifact, muscle artifact, and environmental noise such as varying power line interference. The excess noise complicates detection of myocardial ischemia. This is further discussed in Section 2.2.3.

\subsubsection{ECG Noise Sources}

Given the small magnitude of the cardiac signal, ECG can easily be degraded by the presence of noise. Typical contamination of ECG involves baseline wander, power line coupling (also called 50 or $60 \mathrm{~Hz}$ noise), motion artifact and muscle artifacts [18], [26], [27]. Baseline wander occurs in the bandwidth between 0 and $0.8 \mathrm{~Hz}$ [28] and results from the position of the patient's body, respiration, and changes in electrode impedance (e.g., sweat building up between the electrode and skin) [29]. Power line coupling, which occurs at the same frequency as the power grid (e.g., $50 \mathrm{~Hz}$ in Europe, $60 \mathrm{~Hz}$ in North America) can interfere with ECG recording regardless of CMRR due to impedance differences between electrodes [26]. Motion artifact results from movement of the electrode with respect to the skin [27]. Motion artifact presents particular challenges to ECG signal quality because it can overlap with ECG data in both time and frequency domains. Recall, clinical ECG bandwidth is between $0.67 \mathrm{~Hz}-250 \mathrm{~Hz}$, with the bulk occurring between $1-40 \mathrm{~Hz}$. Motion artifact power typically exists below $5 \mathrm{~Hz}$ [30], however, it can completely overlap with the ECG bandwidth depending on patient movement [31]. Lastly, muscle artifacts, with higher frequency bandwidth (e.g., above 35 $\mathrm{Hz}$ ) occur because of the electrochemical changes affiliated with contractions of muscle 
groups other than the heart [32]. Significant efforts have been made toward mitigation of noise in ECG recordings; these efforts will be discussed in Section 2.4.

\subsection{Ischemia Detection using ECG}

Myocardial ischemia can present asymptomatically, but is usually still detectable via ECG because it affects the repolarization rate of the affected myocardial tissue [33], [34]. This causes current to flow between the ischemic and nonischemic zones, resulting in a shift of the ST segment (seen in Figure 2.4) of a patient's ECG [35], [36].

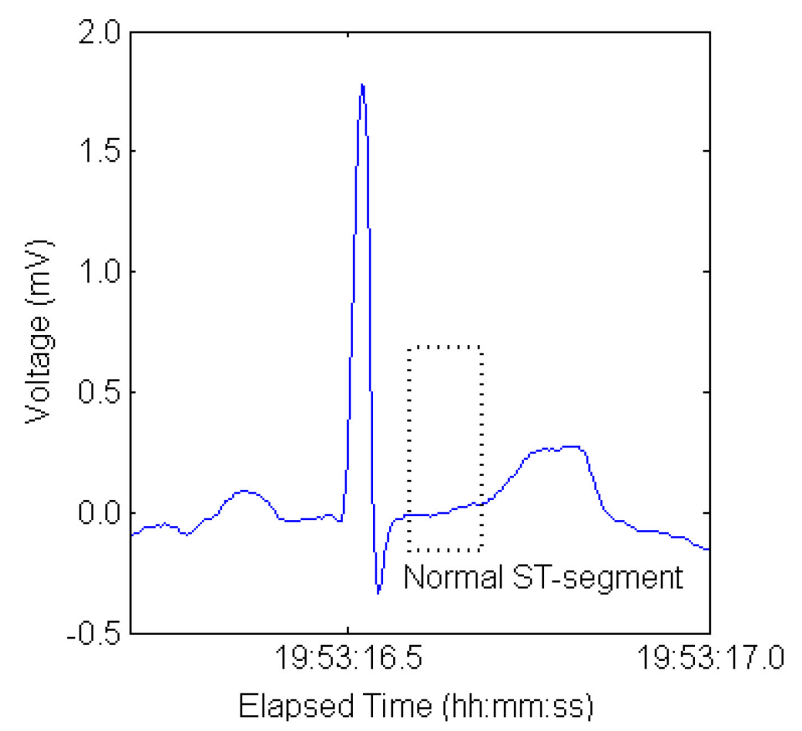

(a)

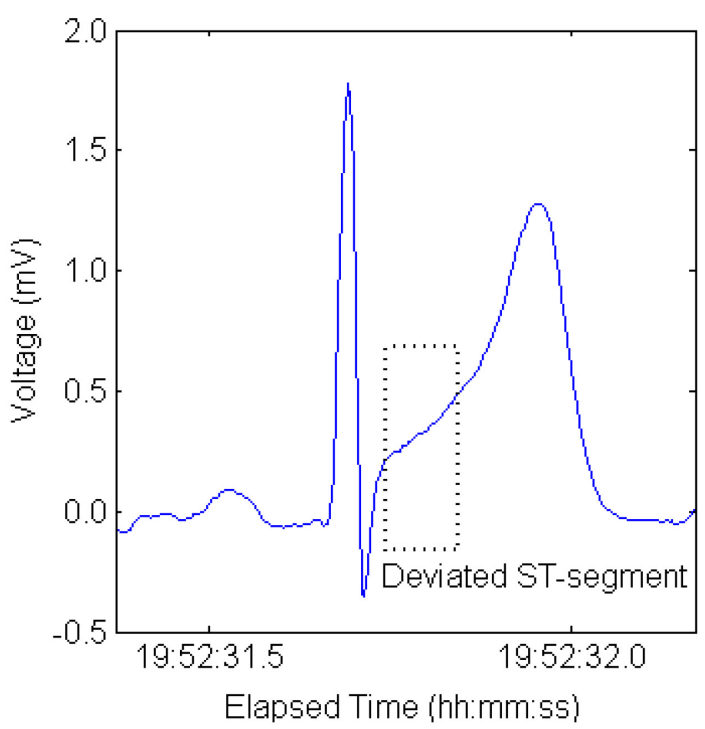

(b)

Figure 2.4 ECG taken from the patient file 's20021' in the Physionet Long-Term ST Database [38], [39]. (a) normal heart beat (b) beat with an ST segment deviation

In normal ECG, the ST segment is typically a plateau (or isopotential) between the QRS complex and the $\mathrm{T}$ wave that is isoelectric with the PR segment, which is between the end of the $\mathrm{P}$ wave and the start of the QRS complex (illustrated in Figure 2.2). During an ischemic episode, the ST segment will deviate from this isoelectric level up to two hours 
before any lasting cell damage occurs [37]. Thus, myocardial ischemia detection via ST segment deviation can provide an intervention window of almost two hours to stop the ischemic cascade before lasting damage occurs.

\subsubsection{Measuring ST segment deviations}

ST segment deviation is a relative measurement. The level of ST elevation or depression is measured relative to the baseline established by the PR segment [36]. This deviation is measured either at the J point (i.e., where the ST segment connects to the QRS complex, as shown in Figure 2.2) or at a specified point between 40 and $80 \mathrm{~ms}$ after the $\mathrm{J}$ point [36]. The PROSE bedside monitors typically use ST measurements derived from the ST level $60 \mathrm{~ms}$ after the $\mathrm{J}$ point (written as $\mathrm{J}+60$ ). As such, this thesis work focuses on $\mathrm{J}+60$ ST measurements [40]. When analysing ST segment deviations, often the slope of the ST segment is also factored in; for example, it is considered normal for there to be an ST segment elevation $60 \mathrm{~ms}$ past the $\mathrm{J}$ point in the V2 lead, provided the ST segment has a positive slope [36].

There are various algorithms to automatically calculate ST segment deviations. These algorithms dictate factors such as how the PR segment is established (e.g. identification of $\mathrm{P}$ and $\mathrm{R}$ waves, averaging multiple PR segments, etc.), and automatic identification of the J point. A commonly used algorithm to analyze the ST segment is the KarhunenLoève transform (KLT) [41], which is used to derive a set of coefficients that describe the ST segment with minimum error. The purpose of these coefficients is to estimate the true form of the ST segment and T wave, absent noise [42], [43]. Commercial systems typically use proprietary methods to analyze the ST segment and these proprietary 
methods are not disclosed. Thus, it is not known how the PROSE commercial monitors estimate ST deviations.

It is worthwhile to note that ECG measurements can be reported both in $\mathrm{mV}$ and in $\mathrm{mm}$. Historically, ECG graph paper used by healthcare professionals was marked in $1 \mathrm{~mm}$ increments where each $\mathrm{mm}$ was equivalent to $0.1 \mathrm{mV}$. The practice of reporting ECG in $\mathrm{mm}$ continues to this day; however, this thesis will refer to ECG and ST deviations using the units of mV only.

\subsubsection{Diagnosis of Ischemia}

Current practice indicates that ST segment elevation at the J point should not exceed 0.1 $\mathrm{mV}$ in any lead, with the exception of V2 and V3 [20]. ST segment elevation at the J point should not exceed $0.2 \mathrm{mV}$ in $\mathrm{V} 2$ and $\mathrm{V} 3$ for men, or $0.15 \mathrm{mV}$ for women [20]. Exceeding these limits may indicate an ischemic episode. Note that while these limits are used to indicate an ischemic episode, ST segment deviations are not solely due to the presence of ischemia; other factors may result in deviations as well [35], as discussed in Section 2.3.3. Typical ischemic episodes are characterized by slow, smooth changes in the ST segment deviation, with a distinct peak [44] in the magnitude of deviation.

To further aid the diagnosis of myocardial ischemia, the various electrical projections of the heart provided by the 12 ECG leads may be employed. It has been found that using a single precordial ECG provides sensitivity of $75 \%$ for detection of myocardial ischemia; using two precordial leads provides sensitivity of over 95\% [45] but current recommendations still suggest using the full 12 standard leads when available [46]. An ST segment elevation in one lead would be reflected as an ST segment depression in a 
lead with a reciprocal view of the heart [20]. However it is important to note that signal strength in the reciprocal lead may not be strong enough for diagnostic purposes [20]. Additionally, for the sake of patient mobility, not all leads are available with ambulatory ECG monitoring.

\subsubsection{Other causes of ST segment deviations}

Ischemia is not the sole cause of ST segment deviations; as mentioned in the previous section, the ST segment can be elevated up to $0.2 \mathrm{mV}$ in V2 and V3 in healthy males. Additional causes of ST segment deviation include hypertrophy, a variety of cardiac and noncardiac drugs, ventricular conduction disturbances and pericarditis (inflammation of the pericardium) [20]. ST deviations can also result from postural changes, for example if a patient lies on their side instead of their back [47].

\subsubsection{PROSE Ischemia Detection}

PROSE uses commercially available ambulatory ECG monitors that stream ECG data wirelessly to a bedside monitor for analysis. Using a proprietary algorithm, the bedside monitor returns an average ST deviation measurement every 5 seconds. These ST deviations are analyzed in real time to generate alarms (following the criteria below), which are sent to clinicians via their mobile device (e.g., smart phone). The signal flow just described is illustrated in the top half of Figure 2.5. This system is designed to enable patient mobility while allowing on-going monitoring. Personal communication with Dr. Homer Yang (Department of Anaesthesiology, TOH) revealed a case where the PROSE monitoring system successfully detected asymptomatic myocardial ischemia, allowing 
for medical intervention prior to infarction [48]; however, this case has yet to be published.

\subsubsection{PROSE Alarm Criteria and False Alarms}

PROSE defines a consistent ST segment depression of at least $1 \mathrm{~mm}$ for 1 minute or longer, a consistent elevation of at least $0.2 \mathrm{mV}$ for 1 minute or longer, or an elevation consistently between $0.1 \mathrm{mV}$ and $0.2 \mathrm{mV}$ for at least 10 minutes to be indicative of myocardial ischemia [5]. ST segment deviations that meet these criteria are associated with a 16 fold increase in relative risk of complication [8].

Issues with ambulatory ECG signal quality have resulted in large numbers of false alarms (i.e. false positives) from the bedside monitors, rendering the ST segment analysis, although effective, clinically impractical. In one test, 146 false alarms were generated from the commercial system over a 48 hour period for a single patient [49]. There were no true alarms during this 48 hour period, as confirmed by manual analysis of simultaneous Holter monitoring by clinicians.

\subsubsection{PROSE White BOX Filtering}

PROSE engineered a 'white box' monitoring system that they operated in parallel with the commercial system to generate custom alerts, as illustrated in the lower half of Figure 2.5. This white box was constructed from a combination of a filtering technique and a rule based algorithm to reduce the false alarm rate [5]. 


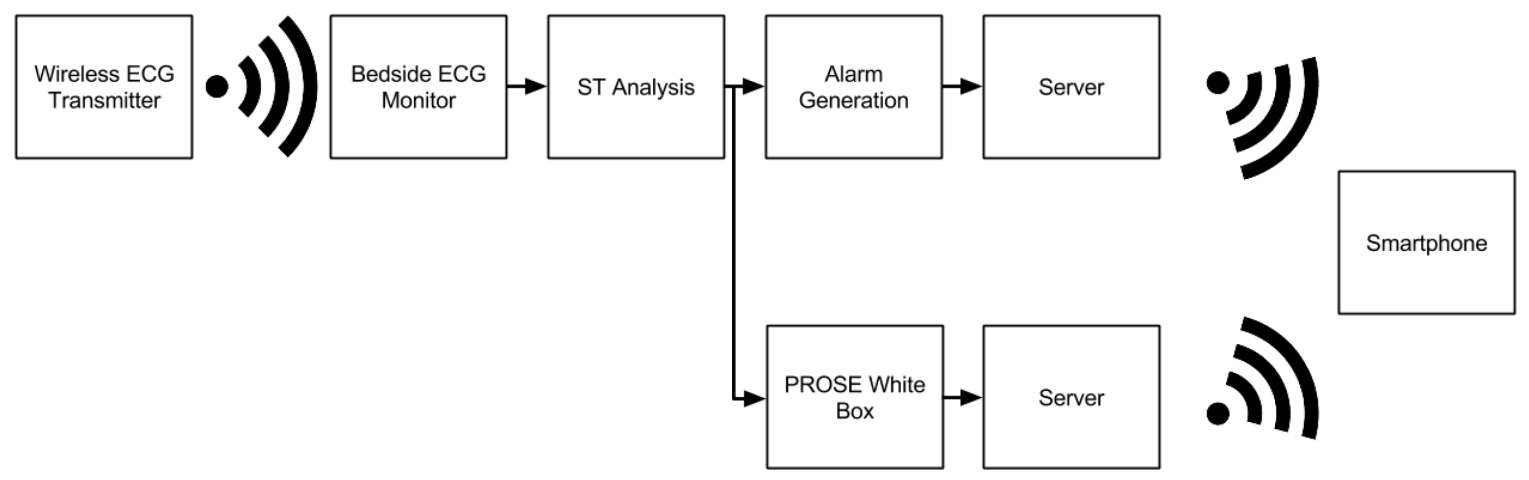

Figure 2.5 Signal flow showing original PROSE set up (top) and parallel white box system (bottom)

In lieu of 146 alarms, this white box approach generated 50 false alarms during the same, previously mentioned 48 hour period [49].

This white box filtering uses sliding window averages to filter out sudden spikes in the reported ST segment deviation, which are likely erroneous measurements [5] resulting from improper identification of the PR segment level or of the J point. The white box rule based algorithm employs a series of logical rules to further enhance the selectivity of alarm generation, such as removing very large, abrupt changed in ST segment deviation likely due to motion artifact (e.g. $+0.9 \mathrm{mV})$ [5].

Despite improvements by the white box, the PPV presented by 50 false alarms generated over 48 hours for a single patient still renders the current PROSE setup clinically impractical. 


\subsection{Biosignal Quality Analysis}

\subsubsection{Rising Need for Biosignal Quality Analysis}

The concept of transmitting medical data to remote locations is not new. In fact, in 1905, shortly after the first ECGs were recorded, they were already being transmitted via telephone cables [50]. However, with advancing technology and wearable sensor systems there has been a much more recent trend toward widespread telemetry. Referred to as 'telehealth', this emerging field promises to improve the quality of medical care both for the aging population and for sparse communities that may not have immediate access to healthcare specialists [51], [52]. Through telehealth, patient data collected at one location may be transmitted and analyzed by technicians remotely, allowing for less intrusive monitoring of patients [52].

In the standard model of care, a trained technician/operator/clinician monitors the data collection process. If signal quality is inadequate for analysis, whether because of excessive noise or improper use of the recording equipment, the technician is able to fine tune system parameters, adjust sensor position or simply re-collect the data. The technician, therefore, acts as a form of quality assurance. As such, the majority of biosignal processing techniques are based on the assumption that the signal is of sufficient quality for mitigation techniques to be effective [52]-[54].

In contrast, data acquisition in telehealth is typically accomplished via unsupervised patient self-management [52]. Thus the quality assurance usually provided by a technician is absent and the lack of a controlled environment introduces potential for set up errors, extraneous motion artifact and additional power line interference [55]. 
Consequently, this presents a need for biosignal quality analysis outside the scope of typical mitigation techniques.

\subsubsection{Categories of Biosignal Quality Analysis}

Biosignal quality analysis can broken into four categories: 1) detection, 2) identification, 3) quantization, and 4) mitigation [56]. As the name suggests, detection is the process of determining whether the biosignal has been contaminated with noise. Identification seeks to establish what type of contaminant is present (e.g., motion artifact, poor electrode contact, quantization noise, etc.). Identifying which type of contaminant is present in the biosignal allows for informed mitigation techniques. Identification can provide direction towards eliminating the source of the contaminant. Quantization estimates the severity of contamination. Quantizing the level of noise present in a biosignal provides valuable insight into the usability of the biosignal. For instance, is the biosignal too contaminated to attempt mitigation or is the level of contamination low enough to ignore altogether? Lastly, mitigation attempts to improve the biosignal quality by removing or reducing contaminants. With the advent of long term recording, mitigation of contaminants may not always be necessary; depending on the application, signal periods where contamination is deemed too severe may simply be discarded from further analysis provided sufficient data remains [52], [55].

\subsubsection{The State of ECG Biosignal Quality Analysis}

Biosignal quality analysis presents a challenge as the source signal is unknown and varies between individual patients [57] - only the observed signal, with an unknown level of contamination, is available for analysis. There are on-going advances in mitigation 
techniques that could help with measurement of the ST segment. For example, [58] highlights how typical baseline removal can distort the ST segment and provides an algorithm based on quadratic variation reduction that preserves the ST segment integrity; however, there is an underlying assumption that ECG signal quality is otherwise adequate. Promising work targeted at mitigation of motion artifact uses variations in the half cell potential to remove motion artifact via adaptive filtering, but this requires additional data channels and specialized electrodes [59].

In this research project, the large time window between the occurrence of detectable ST segment deviations and permanent cell damage means mitigation of poor quality ECG is not needed - it is sufficient to wait for periods of better signal quality. Consequently, mitigation techniques are not being explored in this thesis. Furthermore, the commercial bedside monitor already employs mitigation techniques to improve quality of the ECG signal trace and the commercial system's algorithms are proprietary, thus additional mitigation may cause signal distortion, negatively affecting the system's performance. In fact, it is also not necessary to identify what type of contaminant is present in the signal, just whether or not contamination is severe enough to affect system performance. Thus the focus is on detection and quantization.

Previous work has examined the role of detection and quantization of biosignal quality for ECG but target applications are typically for arrhythmia detection [60]-[63] or employ multiple redundancy measures (such as multiple ECG leads [64], [65], or arterial blood pressure [66], [67]). More recent developments assess the acceptability of ECG recordings on the basis of the recorded ECG alone but these are targeted at mobile 
devices [68], [69] or require training [70]. Autocorrelation is used in [71] to make signal quality scores, however this research is focused primarily on baseline wander. In [72], the authors specifically investigate assessment of signal quality using single-lead ECG recordings. They remove large artifacts using masks for rail contact, high frequency noise and low frequency noise as outlined in an earlier paper [73]. Acceptability of the remaining 'clean' ECG data is then determined through a classifier based on a series of features delineating differences between the ECG under analysis and typical QRS morphology. The authors conclude that biosignal quality analysis of single-lead ECG data is feasible but do not investigate its impact in the clinical setting [72].

Previous work on biosignal quality analysis targeted toward HR or arrhythmias are not directly translatable to ST analysis, which examines long term changes of the ST isopotential; HR detection does not necessarily care about the specific PQRST complex morphology and arrhythmia analysis does not typically focus on the low frequencies affiliated with the ST segment. The methods presented in this thesis provide a metric of biosignal quality that is derived without the need for supporting sensors or data. These methods do not require time to build up a database of known ECG morphology; rather they leverage the repeatability of the PQRST complex to differentiate between ECG signal and noise. Furthermore, the work presented in this thesis steps forward and applies this biosignal quality metric to improve performance of a myocardial ischemia detection system. 


\subsection{Summary}

Myocardial ischemia can be detected up to two hours before lasting cell damage occurs by analyzing changes in the ST segment elevation or depression. POISE found that prophylactic administration of $\beta$-blockers prevented serious cardiac complications following noncardiac surgery but also increased the incidence of bradycardia, hypotension and stroke when administered to all at-risk patients. PROSE seeks to evaluate targeted $\beta$-blocker administration solely to at-risk patients experiencing myocardial ischemia, which can be identified up to two hours prior to permanent cardiac damage (e.g., myocardial infarction, cardiac arrest, cardiac death) by changes in the ST segment of a patient's ECG. Despite efforts to filter erroneous ST segment measurements by PROSE, false alarm rates from a commercially available bedside ECG monitor are too high for clinical practicality. Since mitigation of ECG signal quality is already being performed by the commercial system, this thesis instead investigates quantization of ECG signal quality as a means to improve the PPV of ischemia alarms without detriment to their sensitivity. 


\section{Methodology}

\subsection{Signal Quality Verification System}

The central theme of this thesis is automatic ECG signal quality analysis. This thesis presents methods to automatically quantify signal quality for a single ECG lead, then investigates the possibility of using a parallel system to gate myocardial ischemia alarms. The goal of this parallel signal quality verification system (SQVS) is to increase the PPV of myocardial ischemia alarms while maintaining sensitivity. To accomplish this, the raw ECG signal is automatically assessed to generate a running metric of the signal quality, termed the signal quality index (SQI). The SQI values (SQIs) are then used to adapt the generated alarm outputs.

Commercial entities have been developing ST analysis algorithms over many years, each with their own proprietary system. While a more ideal solution may be obtained for each individual commercial system through detailed knowledge of that system, disclosure of proprietary algorithms is unlikely. This approach is also not feasible for widespread deployment across multiple care centres using various different commercial systems. This thesis work, instead, treats the commercial system as a black box, making the SQVS applicable to a variety of commercial systems already deployed.

Three system approaches are investigated in this thesis. The first approach, $S Q V S_{S T}$, uses SQIs to gate ST deviation measurements, or ST values, between the ST analysis block and the alarm generation stage of Figure 2.5. This approach stops ST values acquired from poor quality ECG from reaching the alarm generation stage. By limiting potentially 
false ST values, the PPV of the alarms may be increased. This approach, illustrated in Figure 3.1, is described in detail in Section 6.1.1.

The second approach, $S Q V S_{E N}$, uses SQIs to enable or disable to the alarm generation station. The alarm generation stage runs on a series of timers as described in Section 3.5. This $S Q V S_{E N}$ approach freezes all operation of the alarm generation stage (including timers), whereas the first $S Q V S_{S T}$ approach allows the alarm generation stage to continue to operate, just without gated ST values being inputted. This second approach, illustrated in Figure 3.2, is described in detail in Section 6.1.2.

The third approach, $S Q V S_{A L}$, uses SQIs to gate generated alarms after the alarm generation stage and before they reach the server for dissemination to clinicians' mobile devices, as shown in Figure 3.3. This third approach, covered in detail in Section 6.1.3, allows all ST deviation measurements through to the alarm generation stage, but gates the alarms themselves if generated during periods of poor signal quality.

PROSE implemented server delivery of alerts to clinicians' smart phones; however, the work completed for this thesis is focused on the acquisition of ST values and alarm generation. Thus, server delivery of mobile alerts was not considered or implemented in this thesis work as it was not directly relevant. The wireless transmitter and bedside monitor (which includes the ST analysis) are described in Section 3.4.3. The alarm generation stage is described in detail in Section 3.5. ECG biosignal quality analysis and gating were all performed in the MATLAB environment, as described in Section 3.4.1. 


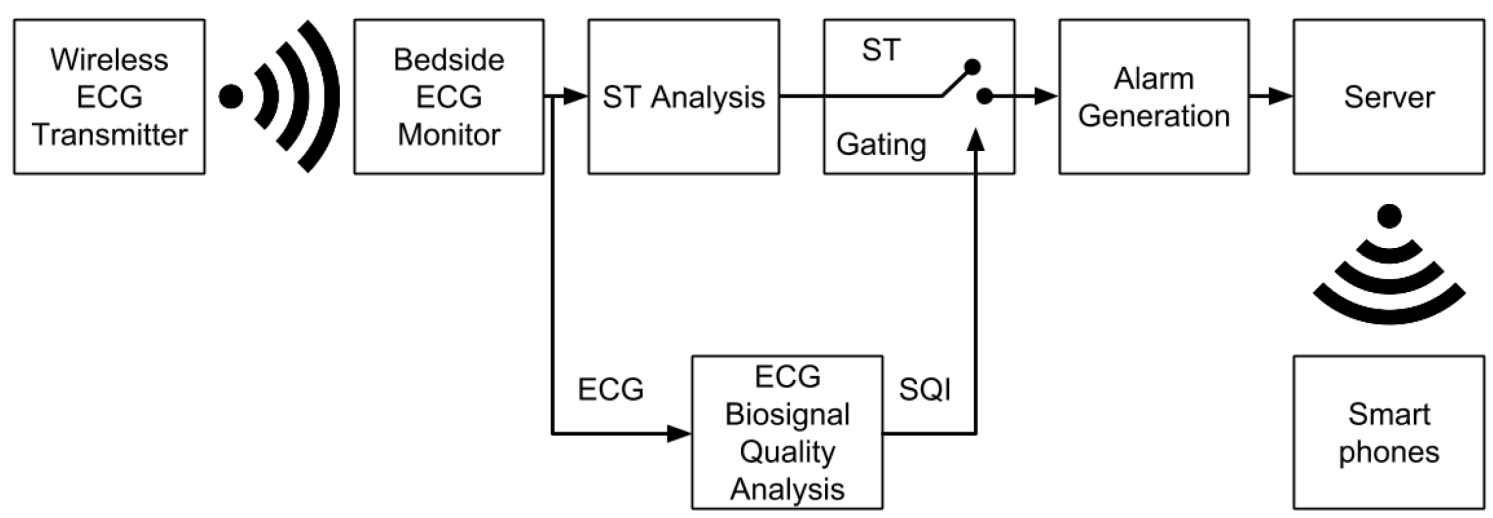

Figure 3.1 Suggested $S Q V S_{S T}$ approach to gate $S T$ values based on poor signal quality before alarm generation

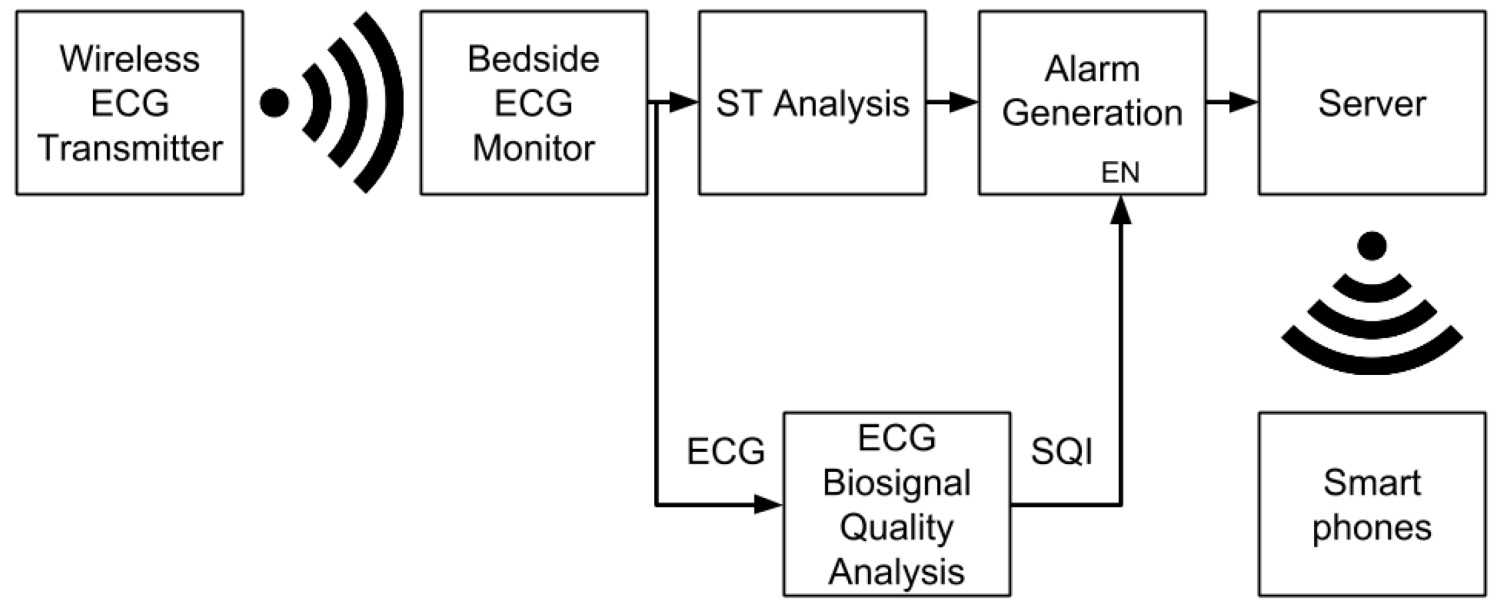

Figure 3.2 Suggested $S Q V S_{E N}$ approach to gate enable/disable the alarm generation

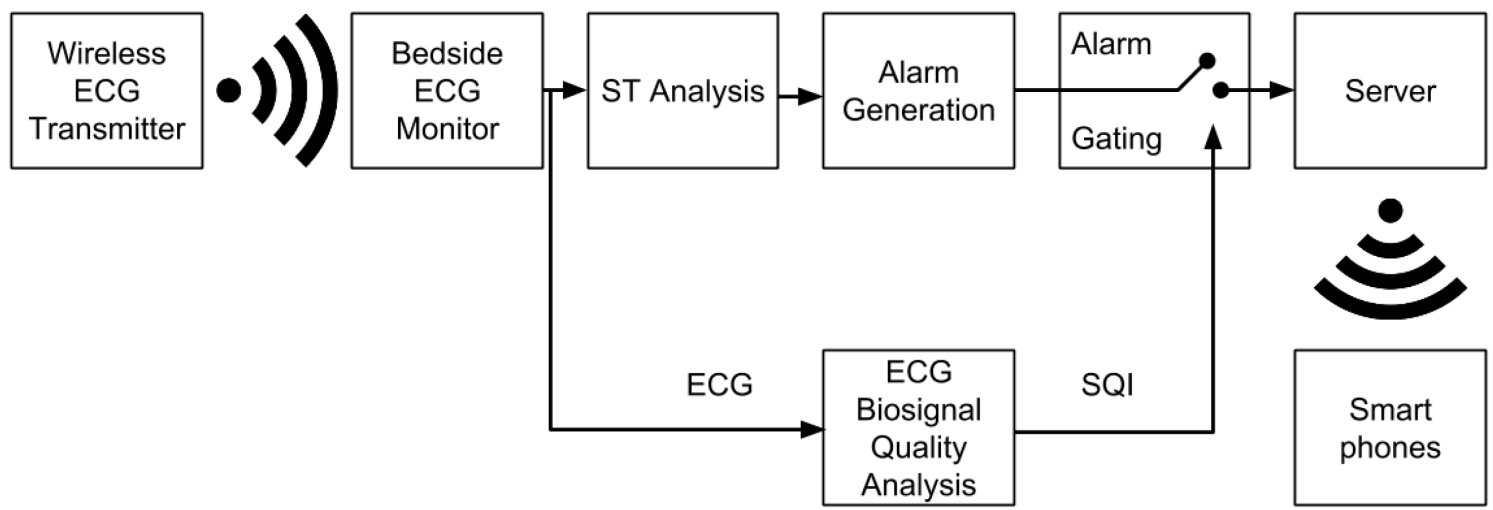

Figure 3.3 Suggested $S Q V S_{A L}$ approach to gate alarms that are based on poor signal quality 


\subsection{Methodology Overview}

The effect that biosignal quality analysis has on the PPV and sensitivity of myocardial ischemia detection was investigated using a collection of long term (e.g., 24 hours) ambulatory ECG records. These ECG records were used to establish and validate the signal quality index (SQI), a metric of ECG biosignal quality (Chapter 4). This metric was validated using both typical ECG data, and ECG data containing ST deviations. This process is described in more detail in Section 3.6.

The causality between ECG biosignal quality and false ischemia alarms was then explored using the previously mentioned ambulatory ECG records and a commercially

available bedside ECG monitor (Chapter 5). This was done to evaluate the feasibility of using SQIs to increase PPV of myocardial ischemia alarms. This process is described in Section 3.7. Finally, the effect of alarm gating based on biosignal quality was examined (Chapter 6). ECG records contaminated with motion artifact were fed through the bedside monitor to generate ST estimates, which were then used to generate ischemia alarms. Ischemia alarms generated from the bedside monitor's ST analysis were gated at various SQI levels and compared to known ischemia alarms in the ECG records as outlined in Section 3.8 .

\subsection{Signal Sources}

The majority of signals used in this thesis are from the Physionet online databases [38]. ECG was simulated to obtain specific HRs as part of the signal synchronization process described in Section 3.4.4. 


\subsubsection{Physionet Databases}

All ST values in this thesis work were derived from ECG data taken from Physionet's online databases. Physionet is a free resource provided by the National Institute of Biomedical Imaging and Bioengineering (NBIB) and the National Institute of General Medical Sciences (NIGMS). Both NBIB and NIGMS are part of the National Institutes of Health (NIH), which falls under the U.S. government's Department of Health \& Human Services. Physionet provides research investigators with free access to a large collection of various physiological signals, removing the need for expensive data collection. Of specific interest to this thesis are the Long-Term ST Database [39] and the MIT-BIH Noise Stress Test Database [74].

\subsubsection{Long-Term ST Database}

The Long-Term ST Database [39] contains 86 ECG records from 80 different human subjects. Records are comprised of ambulatory ECG data containing ischemic episodes and are typically between 20 and 24 hours in length. The majority (68) of these records contain two leads of ECG data - the remaining 18 records have three leads. Lead configurations vary from record to record; some records contain Lead II and V2 data, others Lead III and V4, and still others use the EASI configuration [75] instead of the standardized 12 lead configuration. PROSE monitors patients using Lead II and V5. Consequently, this thesis work was limited to only analyze records containing Lead II and V2 data. These records were chosen because they contained the closest ECG projections to those used by PROSE. In total, 16 records from Physionet's Long-Term ST Database fit these criteria and are listed in Table 3.1. 
Table 3.1 Physionet's Long-Term ST Database records with Lead II and V2 data

\begin{tabular}{|c|c|}
\hline Signal Name & Length (hh:mm:ss) \\
\hline s20011 & $22: 52: 59$ \\
\hline s20051 & $21: 18: 44$ \\
\hline s20061 & $22: 44: 58$ \\
\hline s20071 & $21: 05: 15$ \\
\hline s20081 & $22: 48: 49$ \\
\hline s20091 & $22: 30: 28$ \\
\hline s20101 & $20: 22: 10$ \\
\hline s20111 & $22: 11: 16$ \\
\hline$s 20121$ & $21: 11: 44$ \\
\hline$s 20141$ & $20: 55: 00$ \\
\hline$s 20201$ & $21: 47: 43$ \\
\hline$s 20211$ & $20: 58: 47$ \\
\hline$s 20221$ & $23: 08: 50$ \\
\hline$s 20231$ & $21: 40: 32$ \\
\hline$s 20241$ & $23: 25: 45$ \\
\hline & $23: 22: 01$ \\
\hline
\end{tabular}

Each of the records listed in Table 3.1 was acquired by Physionet using a 12-bit analog to digital converter (ADC) with a sampling rate of $250 \mathrm{~Hz}$ and a gain of 200 units per $\mathrm{mV}$.

\subsubsection{MIT-BIH Noise Stress Test Database}

The motion artifact used to validate the SQI algorithm in Chapter 4, to establish causality between ECG signal quality and false myocardial ischemia alarms in Chapter 5, and for SQVS testing in Chapter 6, is from the MIT-BIH Noise Stress Test Database [74]. The MIT-BIH Noise Stress Test Database contains three 30 minute records of noise. Each of these records has two channels, and was collected by attaching electrodes to the limb of a subject such that the ECG was not visibly discernible. The 'ma' record contains primarily muscle artifacts, the 'bw' record contains primarily baseline wander and the 'em' record contains primarily motion artifact. All three records were acquired using a 12-bit ADC 
with a sampling rate of $360 \mathrm{~Hz}$ and a gain of 200 units per mV. Only the 'em' (motion artifact) record was used to calibrate ECG records to desired SNRs.

\subsubsection{Simulated ECG}

The ST analysis and the biosignal quality analysis are performed on the same ECG data but ST analysis is performed on the bedside monitor, while SQIs are generated on the PC. Synthetic ECG was used to help synchronize the output of the bedside monitor with the SQIs generated on the PC, as explained in Section 3.4.4. Synthetic ECG was generated using 'ecgsyn', a realistic ECG simulator provided by Physionet [76] that allows users to specify HR, among many other parameters. A sample of synthetic ECG generated from the 'ecgsyn' simulator is shown in Figure 3.4

Synthetic signals were generated with HRs of 90 beats per minute (BPM), 60 BPM, and $30 \mathrm{BPM}$ using a sampling rate of $250 \mathrm{~Hz}$. These signals were digitally amplified by 200 units per $\mathrm{mV}$ to match the Physionet Long-Term ST Database records.

Note that synthetic ECG was not used in any analysis aside from alignment of the actual ECG records described in Section 3.4.4, after which it was discarded. 


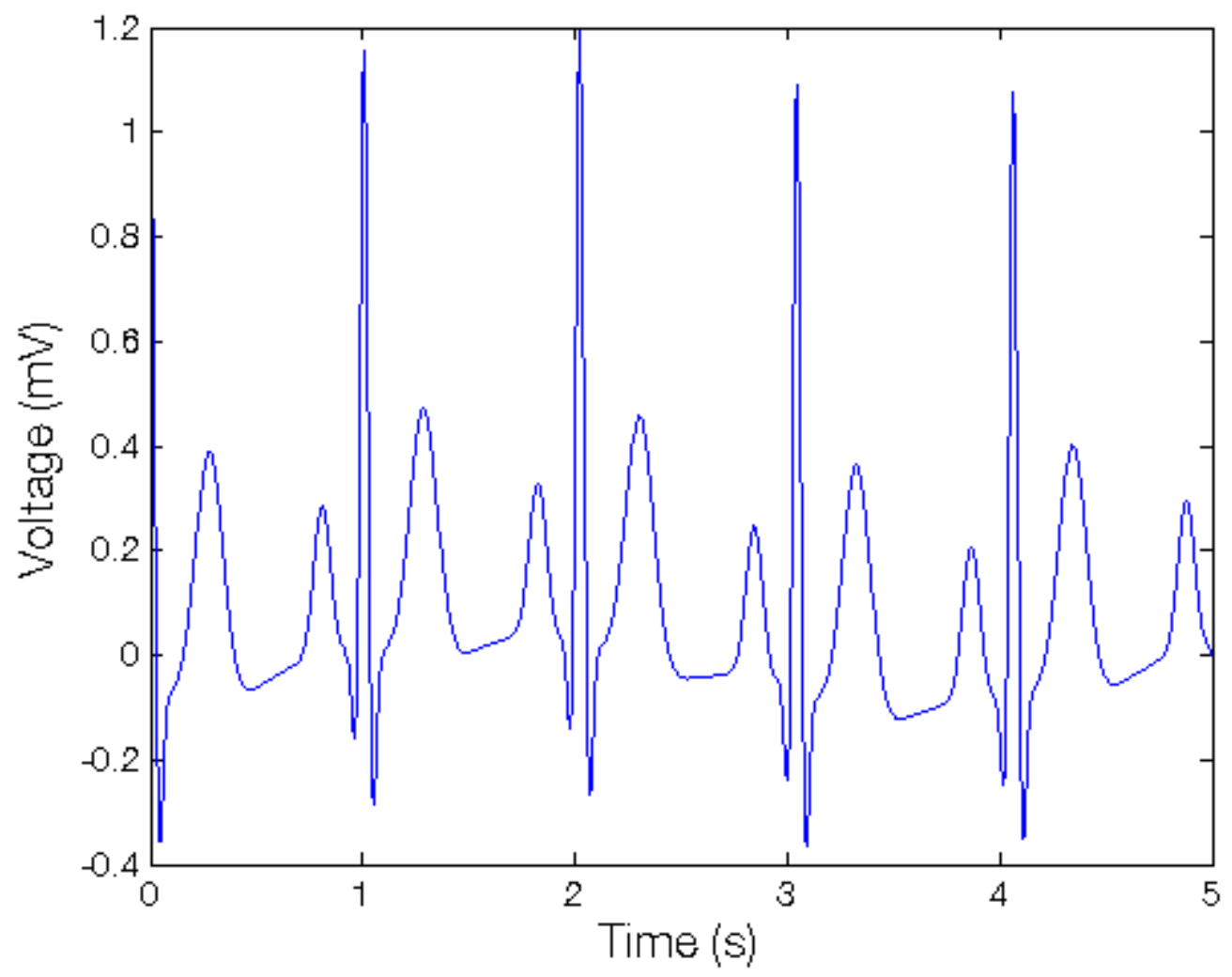

Figure 3.4 Synthetic ECG created using Physionet's 'ecgsyn' simulator

\subsection{Equipment Description}

\subsubsection{Software}

All signal analysis was performed using MATLAB Version 7.10.0 (R2010a), which is a numerical computing environment that facilitates the manipulation and analysis of large data sets. An additional plug in for MATLAB called the 'WFDB Toolbox' was provided by Physionet. This toolbox was used to download ECG records and annotations from Physionet's online databases and import them into MATLAB.

\subsubsection{Data formats}

The ECG records used in this thesis work are stored in three data formats: 
1. Format 212 - primarily used by Physionet. In this format pairs of 12-bit two's complement samples are stored in 3 bytes (note that 1 byte contains 8-bits).

2. Format 16 - the format most accessibly written by the WFDB Toolbox. In this case, 2 bytes are used to store each sample in two's complement format. This format is accepted by the CONTEC Case Conversion Software.

3. CONTEC patient simulator format - data must be converted to this proprietary format in order to be loaded onto the patient simulator (discussed in Section 3.4.3.1) using CONTEC's Case Conversion Software, as described below.

ECG signals must be converted to CONTEC's proprietary format prior to being loaded on the patient simulator. This is accomplished through the use of CONTEC's Case Conversion Software. Case Conversion allows the user to specify the type of signal to be written (i.e., ECG, respiratory or invasive blood pressure) and which format the data is stored in (i.e., 16 or 212), as illustrated in Figure 3.5. CONTEC requires the user to specify which leads of ECG data are present in the record and what the record was sampled at (options are limited to 100, 300, 500 and $1000 \mathrm{~Hz}$ ). Additional required inputs are the baseline value and the calibration (i.e., gain) of the recorded signal. With the exception of MIT-BIH Noise Stress Test Database's 'em' record, all Physionet signals used in this thesis work were sampled with an ADC gain of 200 units per $\mathrm{mV}$ and a sampling rate of $250 \mathrm{~Hz}$. Thus, resampling to $300 \mathrm{~Hz}$ was necessary before loading the records onto the patient simulator. Resampling was accomplished through the use of MATLAB's resample function, which employs anti-aliasing filtering. The required 'baseline' input refers to the ADC zero point, which for Physionet records is 0 . 


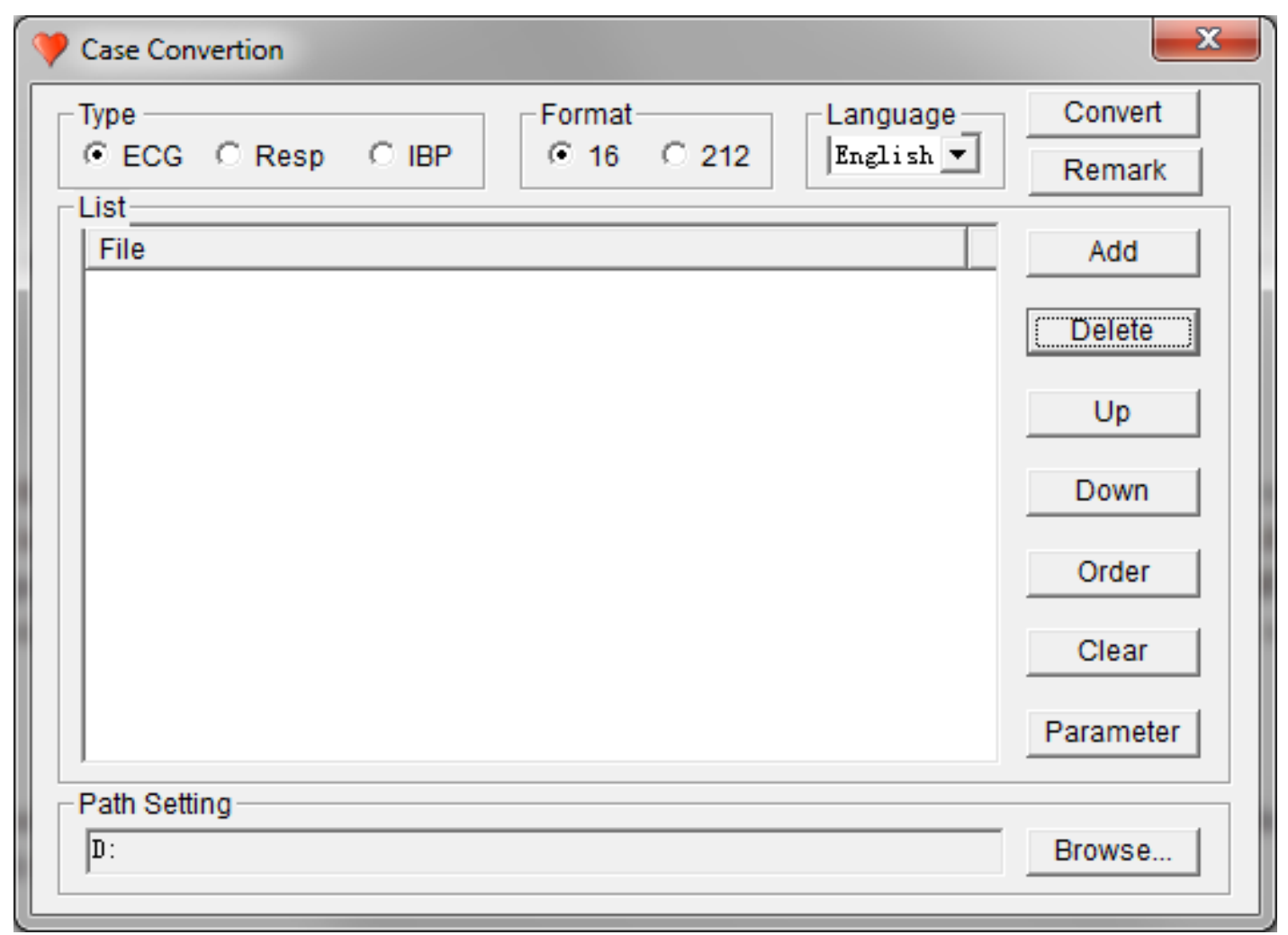

Figure 3.5 Initiating ECG conversion using CONTEC's Case Conversion Software

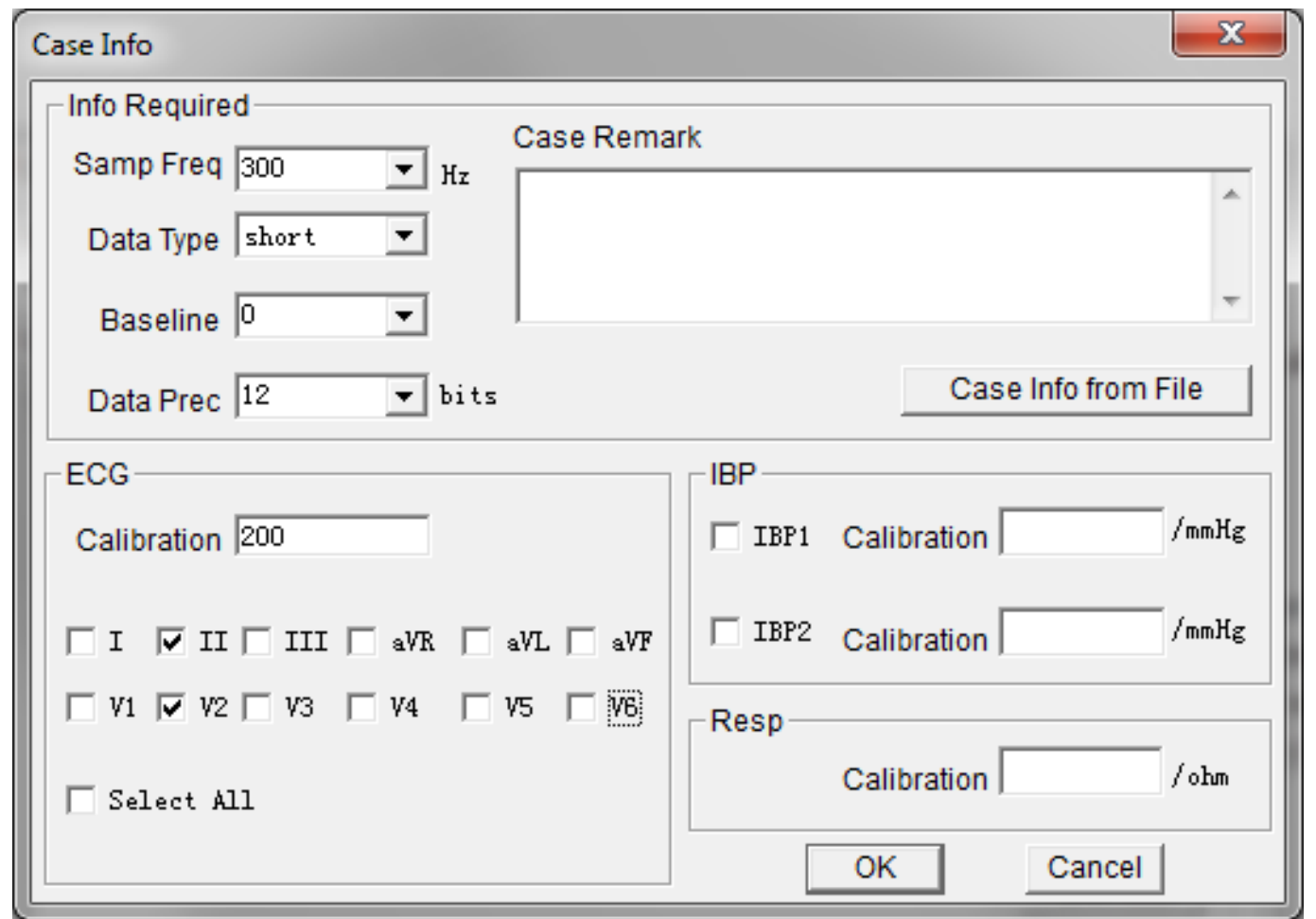

Figure 3.6 Specifying ECG input parameters using CONTEC's Case Conversion Software 


\subsubsection{Hardware}

To test the effect of ECG signal quality with respect to myocardial ischemia alarms, ECG data was played through a patient simulator to a bedside monitor, which performed ST analysis to measure ST deviations.

\subsubsection{Patient Simulator}

The patient simulator, shown in Figure 3.7, is an MS400 Multiparameter Simulator, manufactured by CONTEC Medical Systems, Qinhuangdao, Hebei Province, P.R. China. This device produces varying voltages on a bank of electrical connections that mimic the various projections of a patient's electrical heart activity.

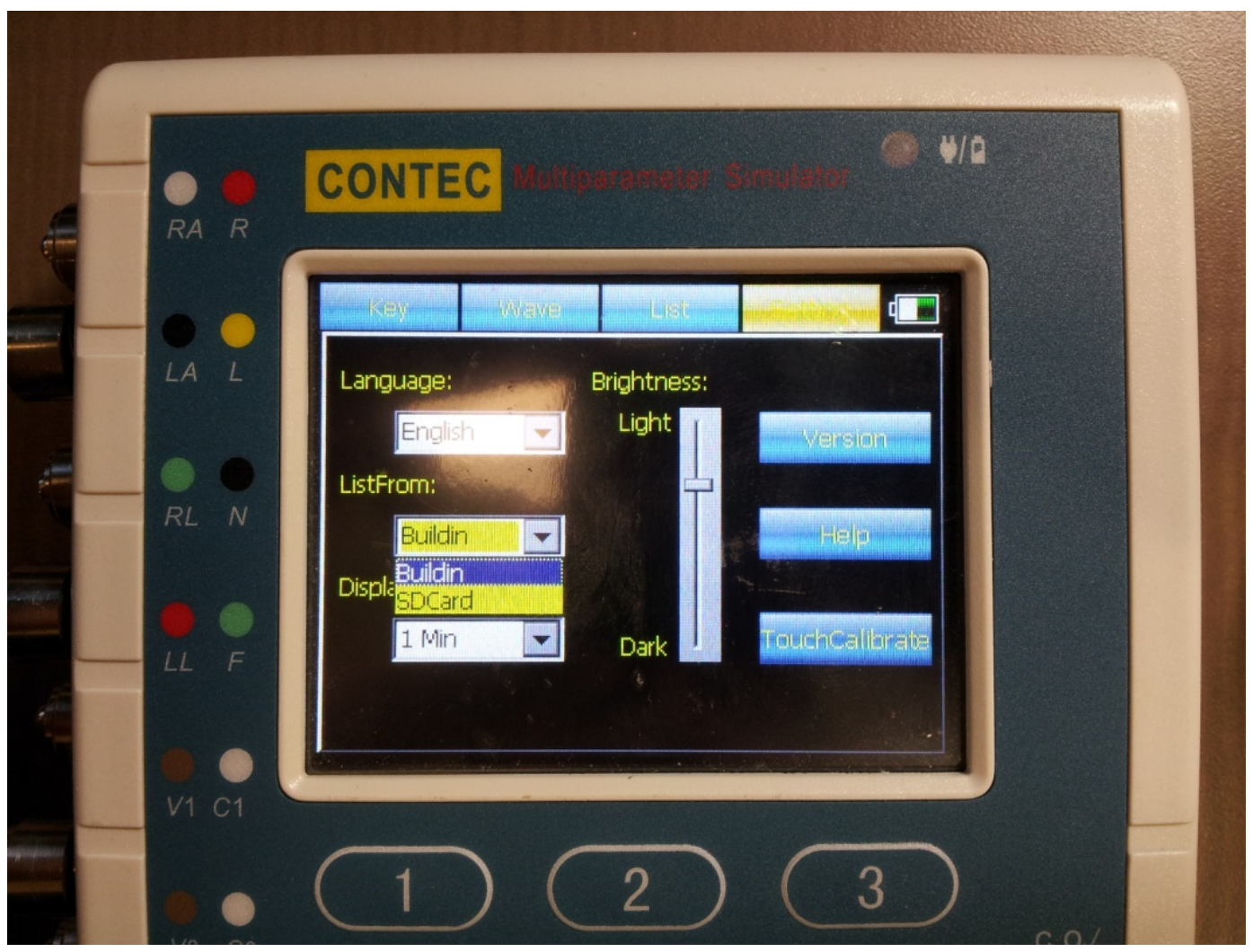

Figure 3.7 MS400 Multiparameter Simulator 
Electrodes connected to the commercial ECG monitor are snapped onto these electrical connections, which are visible on the left hand side of Figure 3.7. The electrical waveforms produced by patient simulator can either be generated internally by the device, or be loaded onto the device via an SD card, using the specialized data storage format described in Section 3.4.2.

Once an ECG record is chosen, the patient simulator continues to loop the record until it is shut off or a new record is chosen.

\subsubsection{Commercial ECG Monitor}

The commercial ECG monitoring system used by PROSE consists of two components: 1) a telemetry unit, and 2) a bedside monitor. Both of these components are manufactured by Spacelabs Medical (Issaquah, WA, USA). Patient electrodes are connected to the wireless telemetry unit, shown in Figure 3.8 (Ultraview Digital Telemetry Multiparameter Transmitter 90345). The telemetry unit transmits the ECG data to a bedside monitor shown in Figure 3.9 (91369 Ultraview SL Bedside Monitor), which performs all calculations on the ECG data (e.g., HR and ST deviation measurement).

The bedside monitor is connected via serial communication (i.e., COM port) to a personal computer (PC) running MATLAB R2010a. The bedside monitor provides an ST value for each ECG lead approximately every 5 seconds to the PC via the COM port. 


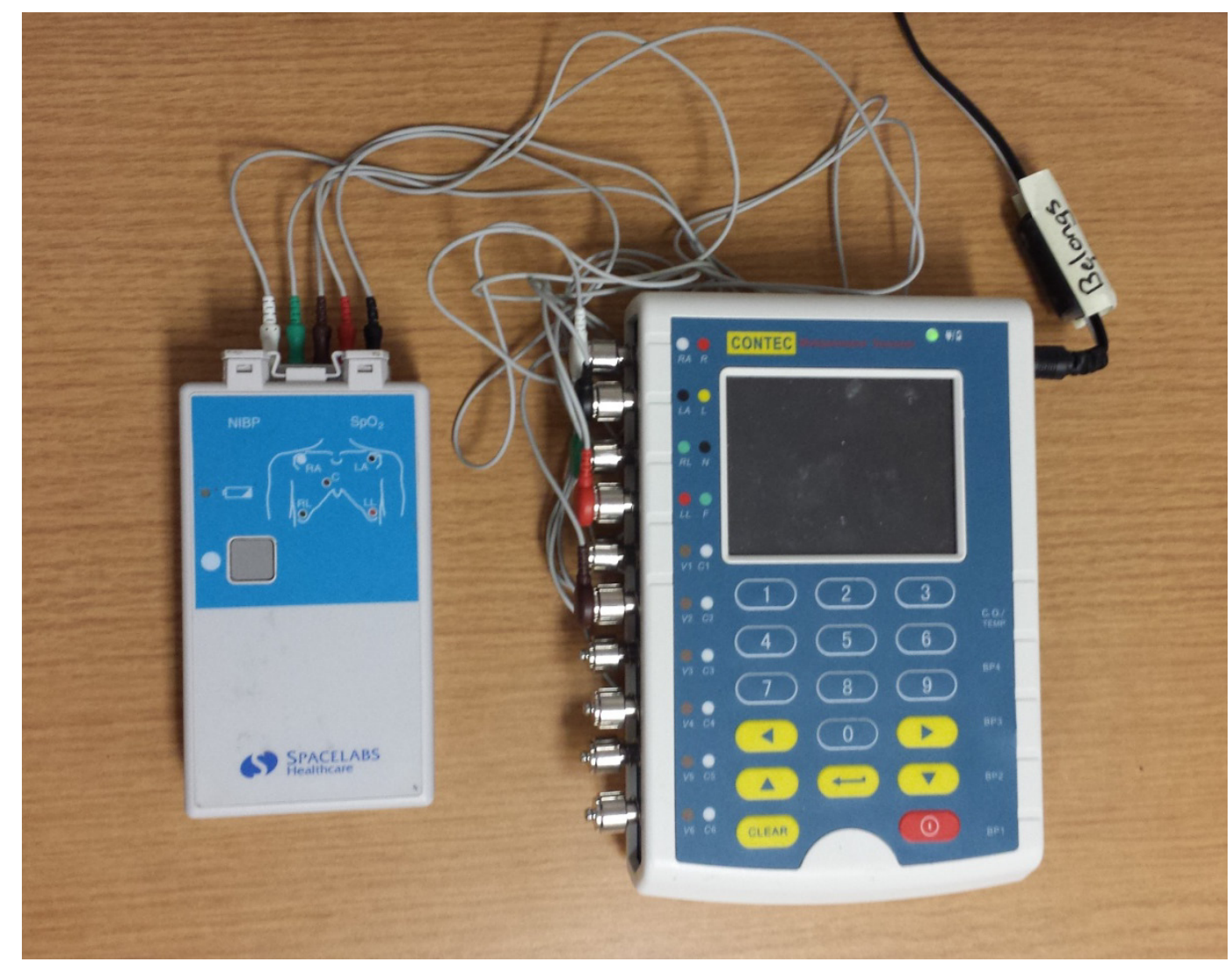

\section{Figure 3.8 Spacelabs Ultraview Digital Telemetry Unit connected to the MS400 patient simulator}

These ST values are accompanied by a value for heart HR and a timestamp indicating the time of day. MATLAB logs these ST values, HR, and timestamp in a text file. Two notable cases for ST deviation logging exist. First, the bedside monitor will not send an ST segment measurement to the PC unless it is able to identify a HR. Thus, during exceptionally noisy periods ST measurement values will not be logged on the PC. Second, if the bedside monitor is able to detect a HR but not able to measure the ST deviation, then it will log an unknown ST value of, '?.??'. 


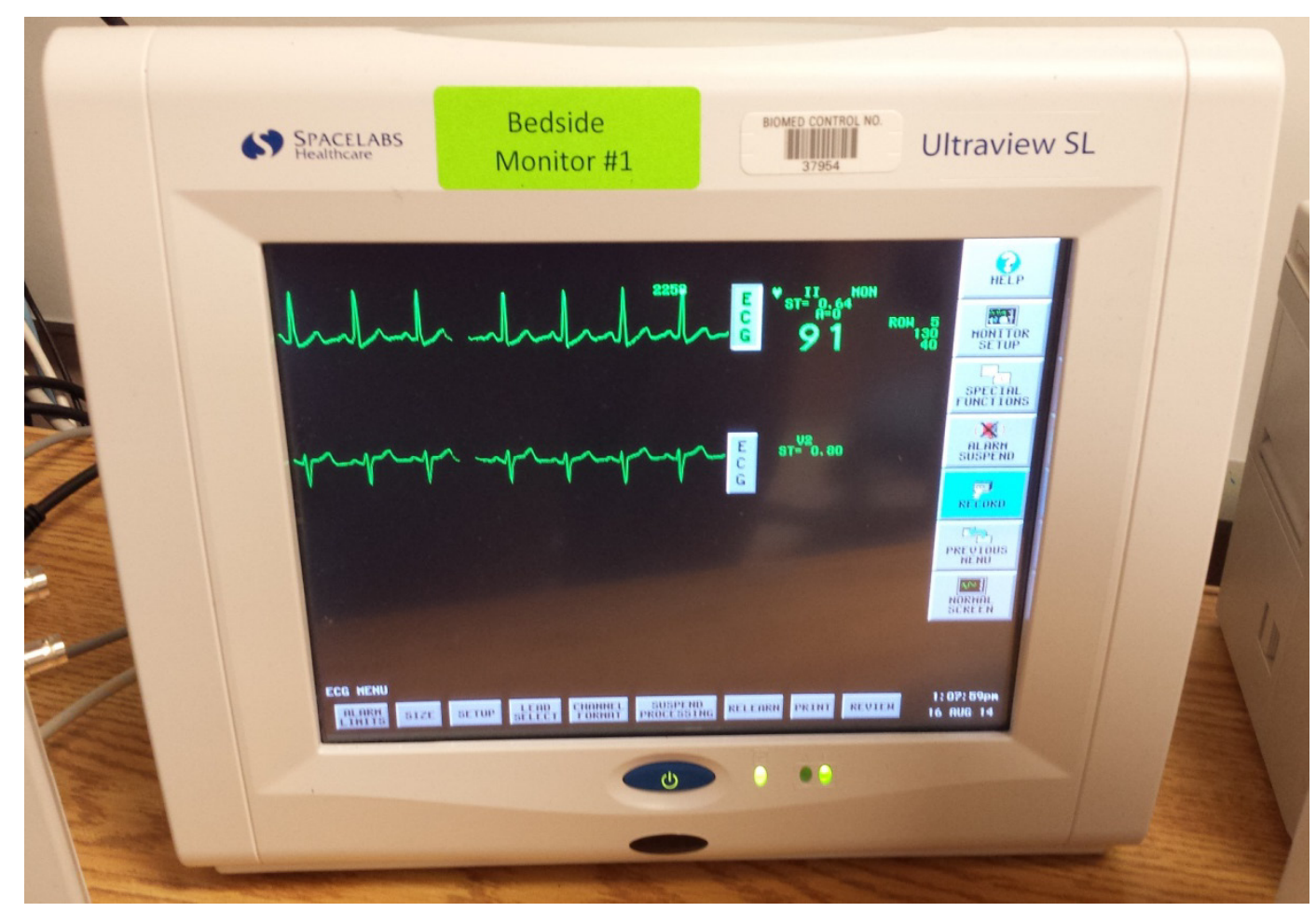

Figure 3.9 Spacelab's Ultraview bedside monitor

Note that the bedside monitor can optionally be linked to a central monitor; however it is the bedside monitor that performs all analysis - the central monitor simply serves as a data aggregator for multiple bedside monitors and thus was not used in this thesis work.

\subsubsection{Synchronizing Signals}

The patient simulator begins streaming the loaded ECG record once selected by the user. These ECG data are transmitted to the waiting bedside monitor, which in turn takes an unspecified amount of time before first logging an ST value on the PC via COM port. The first recorded ST value therefore occurs at an unknown elapsed time from the start of the ECG record. This provides a challenge when trying to synchronize the acquired ST values with the corresponding SQIs, as well as when trying to compare bedside alarms to 
gold standard (GS) alarms derived directly from the Physionet records, as done in Chapter 6.

\subsubsection{Aligning the Start and End of Physionet Data with Logged Bedside Values}

The bedside monitor only transmits information via the COM port when it can detect HR as described in the Section 3.4.3.2 (detected HR can be asystole, or 0 BPM). HR changes are detected almost instantaneously by the bedside monitor while it can take between 30 seconds and 1 minute before an ST value is generated by the bedside monitor. Thus, HR is used to track the start and end of the ECG signal. Synthesized ECG data (described in Section 3.3.2) with set HRs of 90, 60 and 30 BPM were prefixed to start of actual ECG record, while one minute of ECG flat-line (e.g., 0) was appended to the end of the record. In this way, the distinctive 90,60, $30 \mathrm{HR}$ pattern and the period without a $\mathrm{HR}$ are visible each time the record loops on the patient simulator. This allows for easy identification of the first and last logs made by the bedside monitor that correspond to the start and end of the actual Physionet record. Logged measurements outside of these first and last measurements are discarded.

HR values were only used to determine the first and last logged bedside values for an ECG record, after which they were discarded.

\subsubsection{Adjusting Logged Timestamps}

It was found that the patient simulator oscillator crystal had slight calibration tolerances that resulted in varying playback speeds of the loaded ECG records. These tolerances were not severe enough to affect recorded HR values, and had no noticeable effect on ECG morphology; however cumulative effect over 24 hours could result in differences of 
more than 10 minutes between the length of the original Physionet ECG record and the duration indicated by the recorded start and end of logged bedside values. This could introduce an artificial delay when comparing bedside alarms to GS alarms, as well as between the bedside ST values and their affiliated SQIs.

To resynchronize the logged timestamps with the actual Physionet record the following procedure was employed. The oscillator crystal error was assumed to be constant for the length of the ECG record. Thus, an error correction factor, $E r r_{c o r r}$, was derived by comparing the elapsed time (in seconds) of the last recorded sample in the Physionet record and in the bedside logged values as in (3.1).

$$
E r r_{\text {corr }}=\frac{\text { Last Physionet Sample }(\text { in sec })}{\text { Last Logged Bedside Sample (in sec })}
$$

Each timestamp (converted to elapsed seconds) of the logged bedside values was multiplied by Err corr to realign the logged bedside values with the Physionet ECG record.

\subsubsection{Repopulating Gaps in Logged Bedside Values}

As mentioned in Section 3.4.3.2, the bedside monitor does not log any values if it cannot detect HR. Thus during noisy periods it is possible for significant amounts of time to pass without an ST deviation estimate being logged. Timestamps of logged bedside monitor values were analyzed to ensure that there was a logged ST value every 5 seconds. If there was a greater time difference than 5 seconds between two logged values, an ST value of 0 $\mathrm{mV}$ was inserted every 5 seconds to fill the gap. Inserting a non-alarming ST value of 0 $\mathrm{mV}$ was effected to avoid interpreting data gaps that were likely due poor signal quality as alarms, only to then attempt to remove them based on SQIs. In this way, only confirmed ST values generated by the bedside monitor could elicit alarms. 


\subsection{Alarm Generation}

\subsubsection{Implemented Alarm Criteria}

Myocardial ischemia alarms depend both on the magnitude of the ST segment deviation and the duration for which the deviation lasts. PROSE alarm criteria for any single ECG lead require that the ST deviation must be consistently elevated by at least $0.2 \mathrm{mV}$ or consistently depressed by at least $-0.1 \mathrm{mV}$ for duration of at least 1 minute. Alternatively, alarm criteria specify that there should also be an alarm if the ST deviation is consistently elevated by at least $0.1 \mathrm{mV}$ for 10 minutes or longer. These alarm criteria are summarized in Table 3.2. Once triggered, an alarm is considered to be on-going until the ST segment returns to normal levels. Generation of these alarms was accomplished by programming these criteria as a finite state machine using MATLAB.

Table 3.2 Summary of PROSE alarm criteria

\begin{tabular}{|c|c|}
\hline Consistent ST Deviation $(\mathbf{m V})$ & Duration \\
\hline $\mathrm{ST} \geq 0.1$ & 10 minutes \\
\hline $\mathrm{ST} \geq 0.2$ & 1 minute \\
\hline $\mathrm{ST} \leq-0.1$ & 1 minute \\
\hline
\end{tabular}

\subsubsection{Finite State Machine Implementation}

Alarms are generated from ST deviation measurements via a finite state machine (FSM) which analyzes each incoming ST measurement. Each ST measurement has an associated timestamp, indicating the elapsed time since the start of the ECG record. The FSM has 6 dominant states as illustrated in Figure 3.10; one 'resting' state, one 'alarming' state, one 
'cool down' state and three states corresponding to the ramp up times associated with the three criteria listed in Table 3.2. If an incoming ST value meets one of the three criteria listed in Table 3.2, the FSM will log the timestamp of the ST value and enter the corresponding warm up period. If a subsequent ST value is in the normal range (i.e., between -0.1 and $0.1 \mathrm{mV}$ ) the FSM reverts back to the 'resting' state, otherwise the FSM moves into the 'alarming' state if it detects a timestamp greater than the corresponding allotted duration outlined in Table 3.2. The FSM will stay in the 'alarming' state until it receives an ST deviation measurement that falls between -0.5 and $0.5 \mathrm{mV}$. This alarm exit criteria is stricter that the typical 'resting' state limits in order to ensure the FSM does not exit the 'alarming' state until the end of an ischemic episode. The FSM stays in the 'cool down' state until it receives a timestamp that is 30 seconds after it first entered the 'cool down' state. If, while in the 'cool down' state, the FSM receives an ST value that is outside of the 'resting' range (i.e., -0.1 to $0.1 \mathrm{mV}$ ) the FSM will revert back to the 'alarming' state. Again, this 'cool down' period exists to ensure that an alarm is not terminated until the underlying ischemic event is over [39]. Figure 3.10 illustrates these states; state transitions are numbered and summarized in Table 3.3.

It is possible to move between transition states, as illustrated by the horizontal arrows in Figure 3.10. Moving between these states restarts the transition state timers with one exception; it is possible to move back and forth between 'warm up 1' and 'warm up 2' without resetting the 10 minute warm up period affiliated with 'warm up 1'. 
Table 3.3 Summary of transition conditions for the alarm generating FSM

\begin{tabular}{|c|c|}
\hline Transition & Criteria \\
\hline 1 & $-0.1 \mathrm{mV}<\mathrm{ST}<0.1 \mathrm{mV}$ \\
\hline $2 \mathrm{a}$ & $0.1 \mathrm{mV} \leq \mathrm{ST}<0.2 \mathrm{mV}$ \\
\hline $2 \mathrm{~b}$ & $0.2 \mathrm{mV} \leq \mathrm{ST}$ \\
\hline $2 \mathrm{c}$ & $\mathrm{ST} \leq-0.1 \mathrm{mV}$ \\
\hline $2 \mathrm{~d}$ & $0.1 \mathrm{mV} \leq \mathrm{ST}<0.2 \mathrm{mV}(10 \mathrm{minute}$ time not reset $)$ \\
\hline $3 \mathrm{a}$ & $\mathrm{ST}$ timestamp $\geq 10$ minutes \\
\hline $3 \mathrm{~b}$ & $\mathrm{ST}$ timestamp $\geq 1 \mathrm{minute}$ \\
\hline $4 \mathrm{a}$ & $-0.5 \mathrm{mV} \leq \mathrm{ST} \leq 0.5 \mathrm{mV}$ \\
\hline $4 \mathrm{~b}$ & $\mathrm{ST} \leq-0.1 \mathrm{mV} \quad$ OR $0.1 \mathrm{mV} \leq \mathrm{ST}$ \\
\hline 5 & $\mathrm{ST}$ timestamp $\geq 30$ seconds \\
\hline
\end{tabular}

The alarm generating block shown in Figure 3.1 to Figure 3.3 is only considered to be alarming when the FSM is in either the 'alarming' state or the 'cool down' state. For every ST value that enters the alarm generation stage, the FSM will generate a similarly time stamped alarm indicator.

The entire duration from the time that the FSM starts to output an alarm until the FSM returns to the resting state is all quantified as a single alarm. 


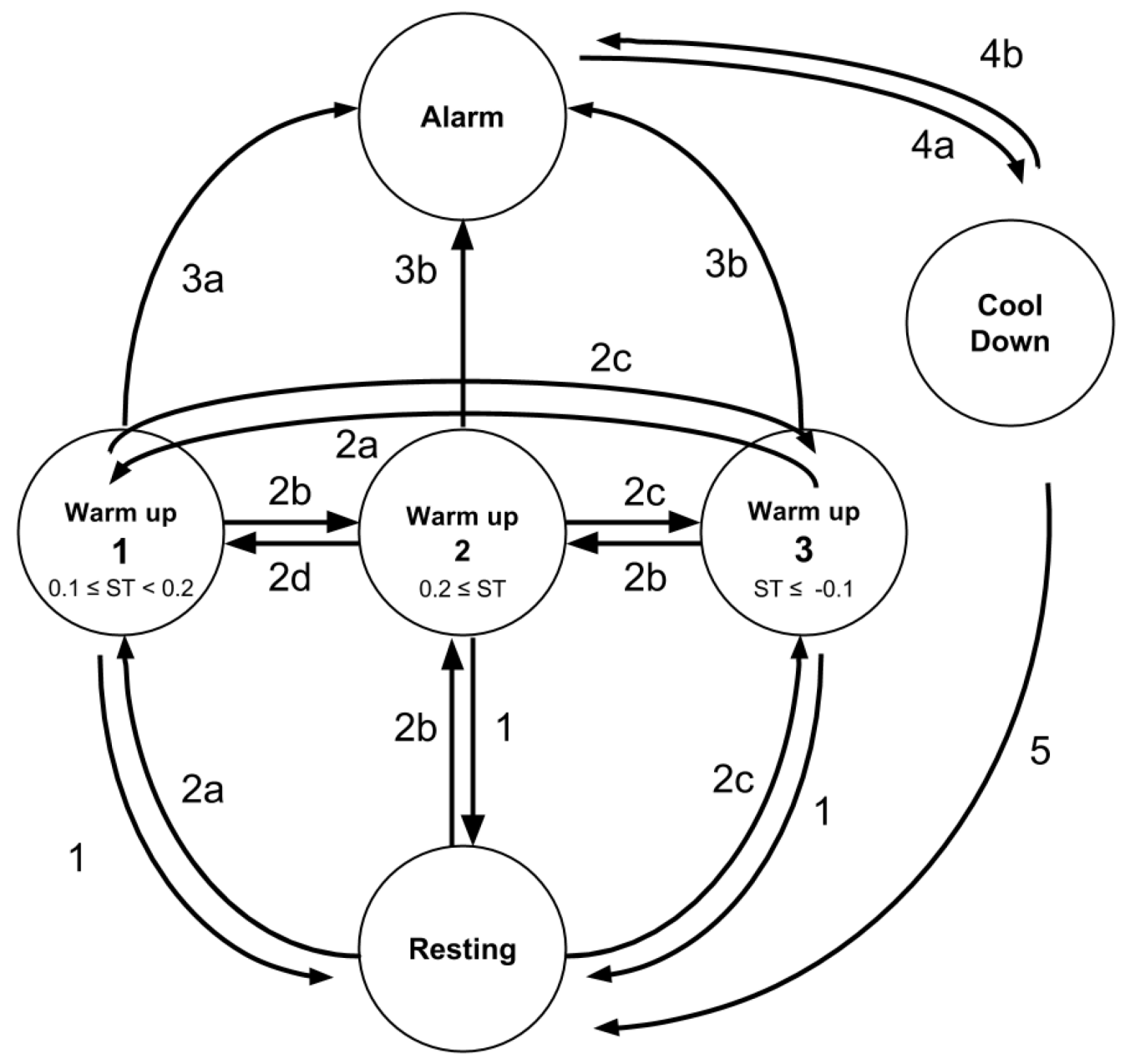

Figure 3.10 Alarm generating FSM. See Table 3.3 for summary of state transition criteria

\subsection{Validating the SQI metric}

To validate the SQI generation algorithm, ECG and motion artifact data were first downloaded from Physionet's Long-Term ST Database and the MIT-BIH Noise Stress Test Database. Signal power for both the ECG and the motion artifact were calculated as described in Section 4.2.2. The motion artifact was scaled and added to the ECG such that the resulting signals had calibrated SNRs. SQIs were then generated for these 
resulting signals and compared to the calibrated SNR levels in order to assess correlation. This validation procedure was performed using both typical, healthy ECG and ECG containing ST deviations affiliated with myocardial ischemia.

This thesis work treats motion artifact as additive noise. It is possible for motion artifacts to affect ECG in other ways as well, such as signal distortion; however, such distortions would result in a lower SQI, which would only further distinguish periods of poor quality. Thus from a practical perspective, treating motion artifact as additive noise is adequate. Determining signal powers, scaling and adding motion artifact, generating SQIs, and comparing the SQIs and calibrated SNR was all performed in the MATLAB environment. Validating the SQI algorithm is discussed in detail in Chapter 4.

\subsection{Investigating Effects of Poor ECG Signal Quality on Alarms}

Chapter 5 investigates the effect of poor signal quality on alarm generation. To do this, ECG and motion artifact data were first downloaded from Physionet's Long-Term ST Database and the MIT-BIH Noise Stress Test Database. The ECG data were selected such that they did not contain any ST deviations. These data were converted to the CONTEC format, loaded onto the patient simulator, and played through the bedside monitor. The same ECG data were then contaminated with motion artifact, converted, loaded onto the patient simulator, and played through the bedside monitor again. The ST

deviation measurements between the clean and contaminated copies of the ECG data were compared. 


\subsection{Investigating SQVS based Alarm Gating}

Chapter 6 examines the impact of using the SQVS to modify alarms based on ECG quality. This was investigated by first downloading ECG records from Physionet's LongTerm ST Database. The ST measurement annotations (saved in Physionet's '16a' annotation files) for these ECG records were analyzed automatically for ischemia alarms using the alarm generating FSM. Questionable alarms were verified by three anesthesiologists at TOH to establish a GS. These ECG records were then contaminated with simulated motion artifact, converted to the CONTEC format, loaded on the patient simulator, and played through the commercial system. SQIs were generated directly from the Physionet records and used to modify the alarm output as illustrated in Figure 3.11 to Figure 3.13. The resulting alarms were compared to the GS alarms as described in Section 6.2.2. Figure 3.11 to Figure 3.13 show the test setups implemented in Chapter 6 for evaluating each of the three SQVS configurations. The dissemination of alarms via server was not needed or implemented for this thesis work, and is consequently grayed out. The point at which ST values and SQIs are synchronized (described in Section 3.4.4) is indicated in these figures by a green dotted line. 


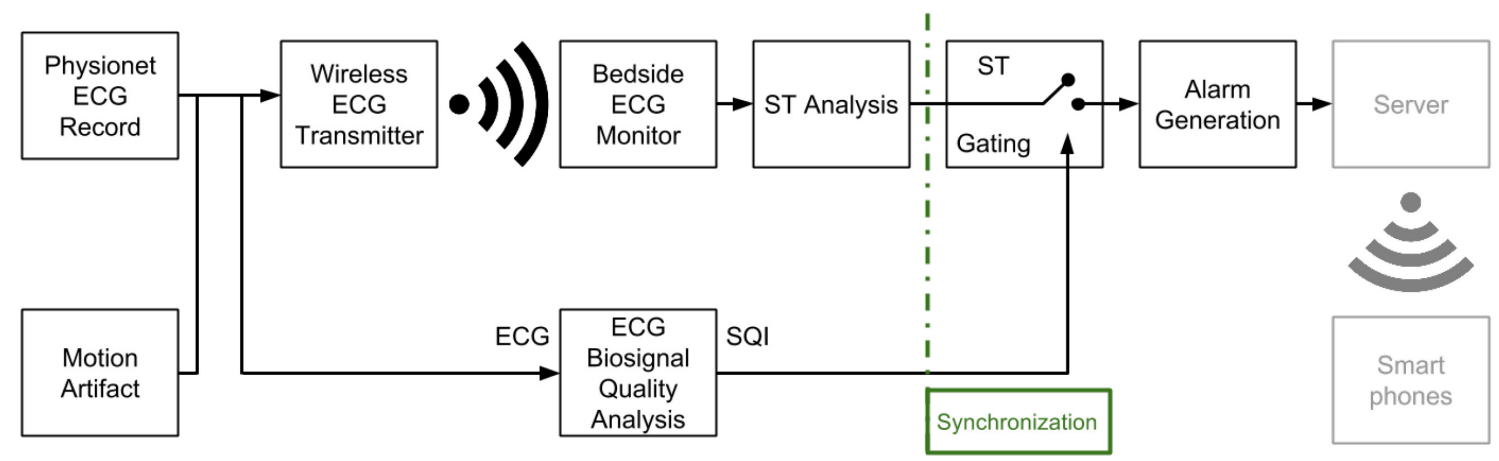

Figure 3.11 Implemented test setup for investigating the effects of using $S Q V S_{S T}$ to gate ST values. Grayed out portions were not implemented in this thesis

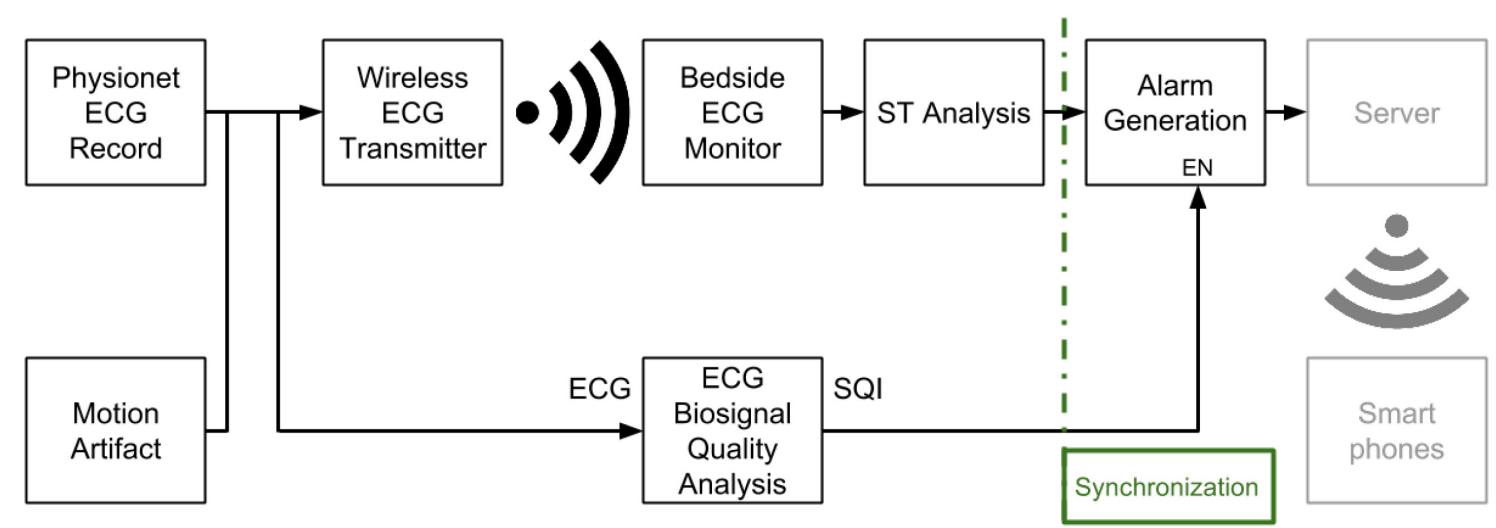

Figure 3.12 Implemented test setup for investigating the effects of using $S Q V S_{E N}$ to disable the alarm generating FSM. Grayed out portions were not implemented in this thesis

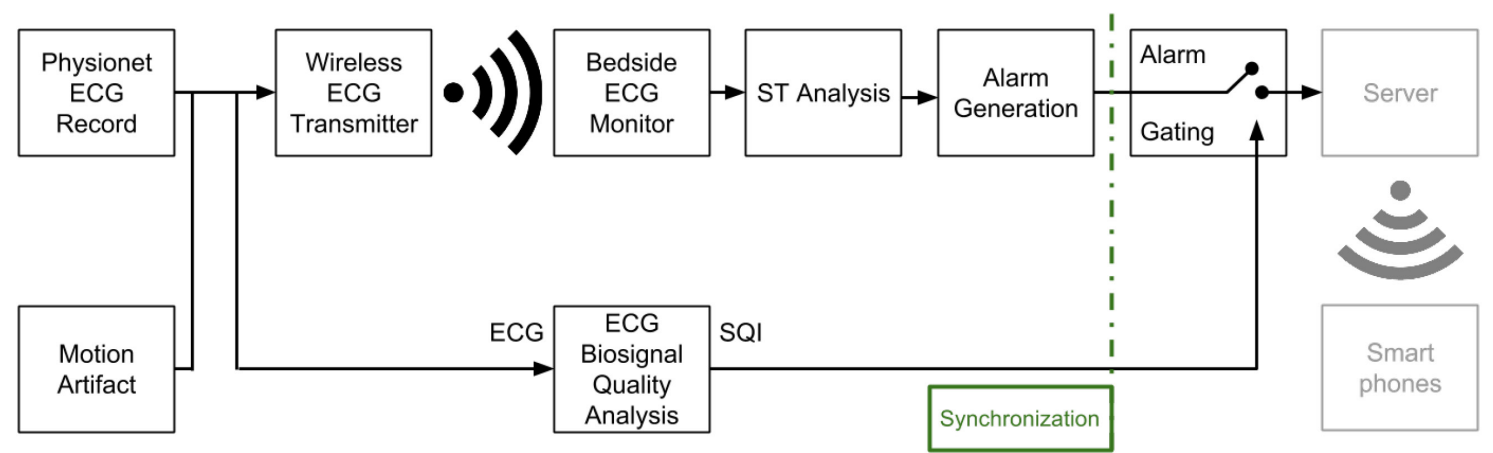

Figure 3.13 Implemented test setup for investigating the effects of using $S Q V S_{A L}$ to gate alarms. Grayed out portions were not implemented in this thesis 


\section{Signal Quality Index}

PROSE uses ambulatory ECG to monitor patients for myocardial ischemia to allow for patient mobility during the recovery period. Ambulatory ECG, however, presents challenges to signal quality due to the presence of motion artifact that ultimately results in frequent false positive identification of myocardial ischemia, rendering it clinically impractical [5].The ability to identify data of poor quality allows gating of the resulting potentially false alarms. This chapter describes the SQI, which provides a method to quantitatively score the signal quality.

\subsection{SQI Generation}

\subsubsection{Preprocessing}

Prior to determining SQIs, ECG signals are digitally high pass filtered to remove any baseline drifts. A zero-phase forward and reverse third order digital Butterworth filter with a $0.67 \mathrm{~Hz}$ cut-off frequency was used, which is currently the American Heart Association's (AHA) recommended method for low frequency noise removal [20]. ECG records in Physionet's Long-Term ST Database are sampled at $250 \mathrm{~Hz}$. Thus, Table 4.1 lists the filter coefficients, $a_{k}$ and $b_{k}$, for this high pass filter at a sampling rate of $250 \mathrm{~Hz}$.

\section{Table 4.1 High pass filter coefficients for pre-processing ECG data prior to SQI generation}

\begin{tabular}{|c|c|c|}
\hline $\mathbf{k}$ & $\mathbf{a}$ & $\mathbf{b}$ \\
\hline 0 & 1.000 & 0.9833 \\
\hline 1 & -2.9663 & -2.9499 \\
\hline 2 & 2.9332 & 2.9499 \\
\hline 3 & -0.9669 & -0.9833 \\
\hline
\end{tabular}


Figure 4.1 shows the zero phase magnitude response for this high pass filter. This preprocessing step is only done in the parallel ECG biosignal quality block - the commercial bedside monitor has its own proprietary signal conditioning methods.

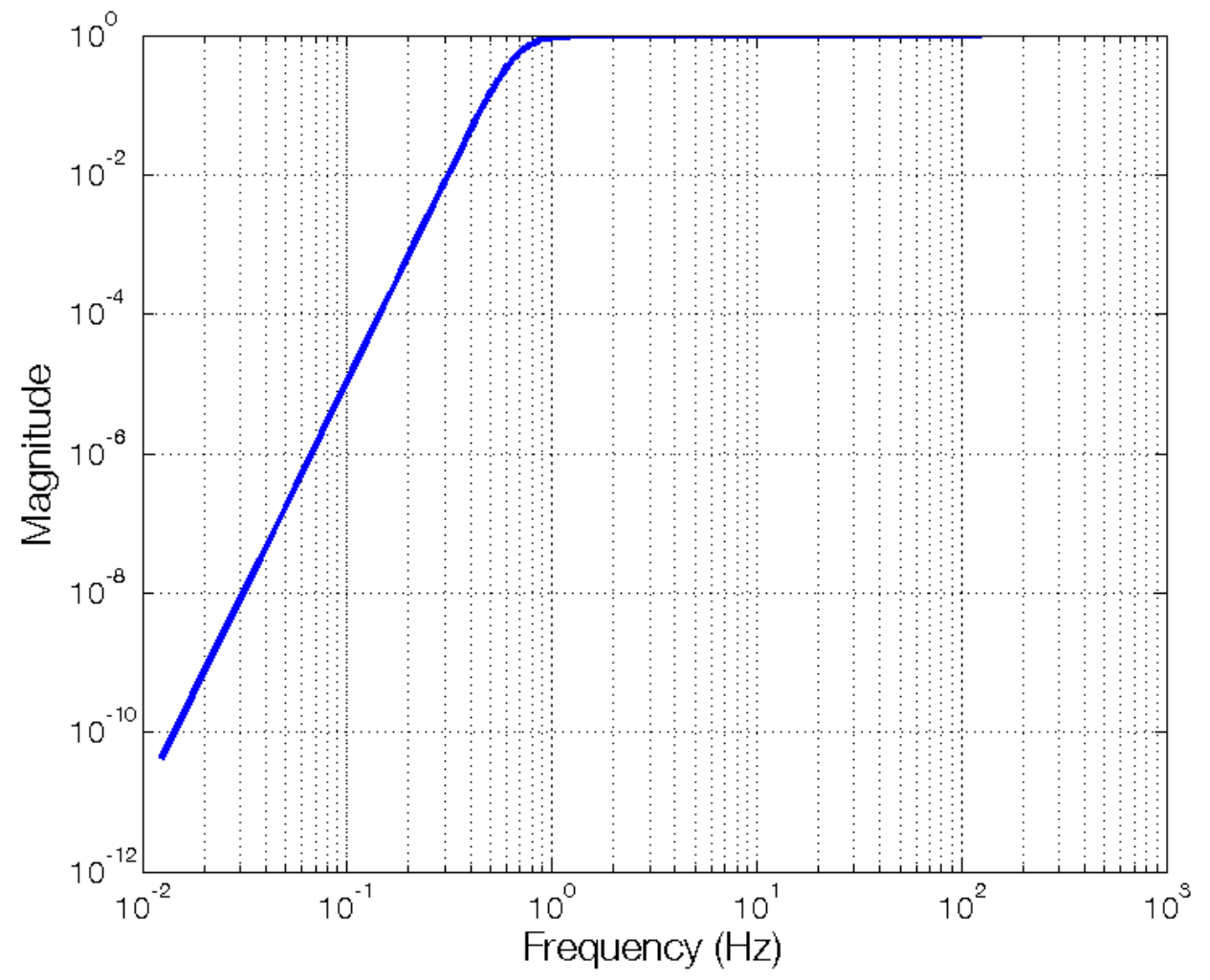

Figure 4.1 Zero phase magnitude response of high pass filter used for preprocessing during SQI generation

\subsubsection{Establishing Analysis Windows}

The Spacelabs commercial bedside monitor has been configured to transmit an ST value via a built-in COM port every 5 seconds to a PC, which records the ST value (refer to Section 3.4.3.2). While the bedside monitor's ST estimation algorithm is proprietary, it is documented in the Spacelabs Ultraview SL user manual [77] that the ST value is based 
on the previous $30-60$ seconds of ECG data. SQIs are generated every 5 seconds and each SQI is based on the previous 30 seconds of ECG data so as to match the bedside monitor's ST estimation algorithm while still being responsive. Thus ECG data is analyzed using a 30 second sliding analysis window that is updated every 5 seconds.

\subsubsection{QRS Detection}

The SQI discussed in this chapter relies on proper identification and alignment of PQRST complexes within an analysis window. The $\mathrm{R}$ peak provides a very precise landmark in the PQRST complex. Thus, proper identification of the QRS complex and subsequently the R peaks is crucial for SQI functionality. Multiple methods exist for real-time QRS detection such as Pan Tompkins [22], Multiplication of Backward Differences (MOBD) [78] or using wavelet coefficients [24]. It is worthwhile to note that QRS detection algorithms may fail during periods of extremely poor signal quality, resulting in misidentified $\mathrm{R}$ peaks. These misidentified $\mathrm{R}$ peaks would result in a low SQI, which from a practical point of view, is representative of the signal quality nonetheless (i.e., if QRS detection isn't possible, the signal quality is probably poor).

This thesis work did not employ QRS detection but used the R peak annotations provided with the ECG records from Physionet's Long-Term ST Database. This is further explained in Section 4.2.3.1.

\subsubsection{Aligning R Peaks}

Within a 30 second analysis window, each PQRST complex (i.e., each heart beat) is isolated into a 0.7 second segment, $W_{m}[n]$, which is centered on the R peak. $W_{m}$ is the $m^{\text {th }}$ complete beat in the analysis window and $n$ represents the individual sample number $(\mathrm{n}=$ 
$\{1,2, N\}$ ). For a sampling of $250 \mathrm{~Hz}$, this corresponds to segments of $N=176$ samples; 87 from before the R peak, the R peak itself, and 88 samples after the R peak. The first and last beats in the analysis window are checked to ensure they have the necessary number of samples before and after the R peak. Incomplete PQRST complexes are excluded from the analysis.

\subsubsection{Ensemble Averaging}

The action potentials that cause the heart to beat are all-or-nothing phenomena; the electrochemical changes occurring within the tissue will, once triggered, always react in the same way [18]. This means that each time a subject's heart beats the resulting PQRST complex should be identical. This repeatability can, in fact, be used for biometric identification, much like an individual's fingerprint [57]. Small morphological changes to the PQRST complex do occur in healthy ECG because of HR changes, such as shortening of the ST segment [79]; however, given the relatively short length of analysis windows (30 seconds), these changes are assumed to be negligible. Morphological changes to the ST segment due to ischemia are likewise considered negligible because of the slow nature of their change [3]. Thus, the ensemble averaged PQRST template should represent the ideal PQRST complex, assuming noise to be uncorrelated [80].

Once all PQRST complexes have been segmented and aligned following the description in the previous section, each of the 176 samples is averaged across all beats to get an ensemble averaged PQRST template, $W_{\text {avg }}[n]$, as described in (4.1).

$$
W_{\text {avg }}[n]=\frac{1}{M} \sum_{m=1}^{M} W_{m}[n]
$$


where $M$ represents the total number of complete beats in the analysis window. Part (a) of Figure 4.2 illustrates ensemble averaging all complete PQRST complexes in 30 seconds of clean ECG data, while ensemble averaging of noisier ECG data is illustrated in part (b).

Following the assumptions that the ideal PQRST waveform does not vary during the analysis window and that the noise is uncorrelated, the SNR improvement provided by ensemble averaging is the square root of $M$ (the number of complete beats in the analysis window) [81]. Note that $M$ varies between analysis windows, and is dependent on the heart rate. As with any statistic, the greater the sample size (i.e., $M$ ), the more closely the ensemble averaged estimate reflects the true PQRST complex. Thus, a slow heart rate will result in lower SNR improvement when obtaining $W_{\text {avg }}[n]$; however, this effect is relatively low provided $M>20$ beats. Using an analysis window of 30 seconds ensures that $M>20$ for heart rates as low as $40 \mathrm{BPM}$. 


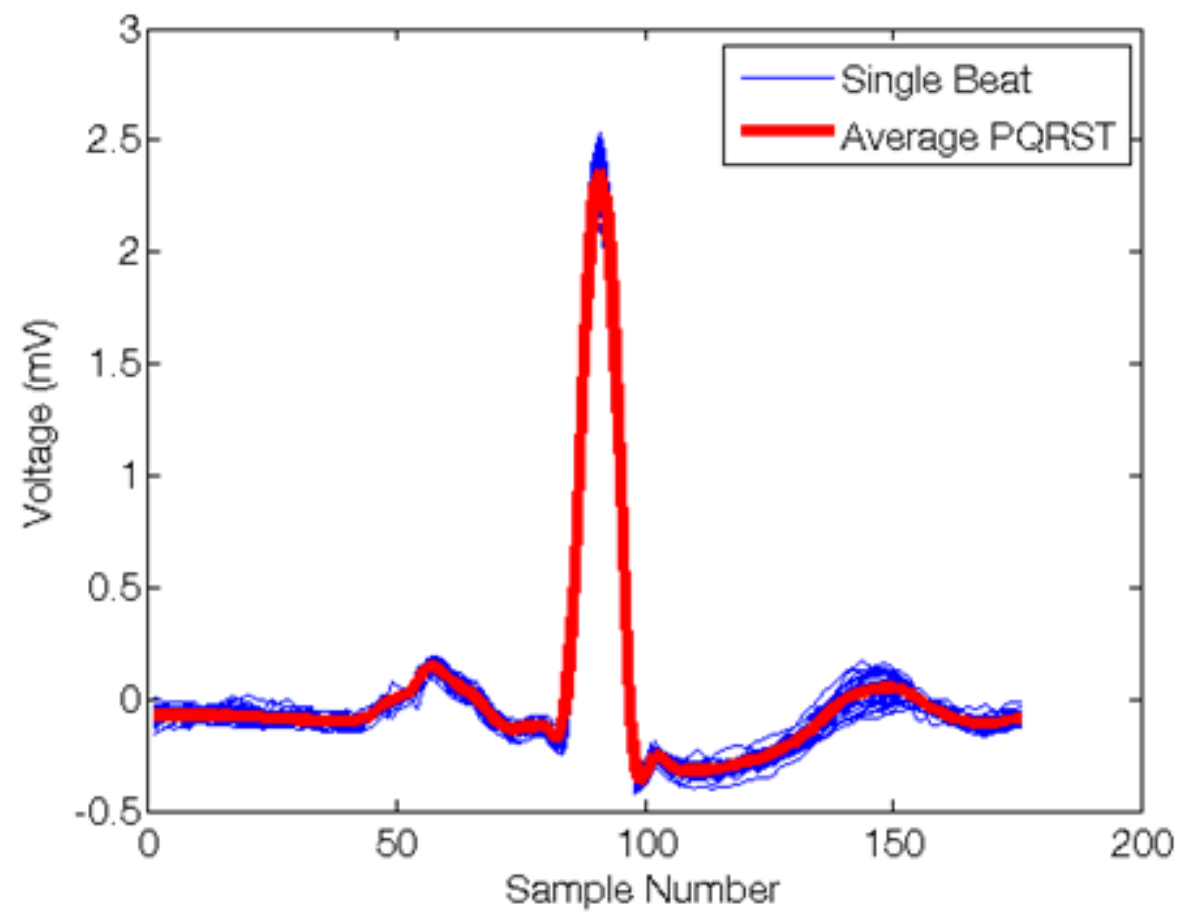

(a)

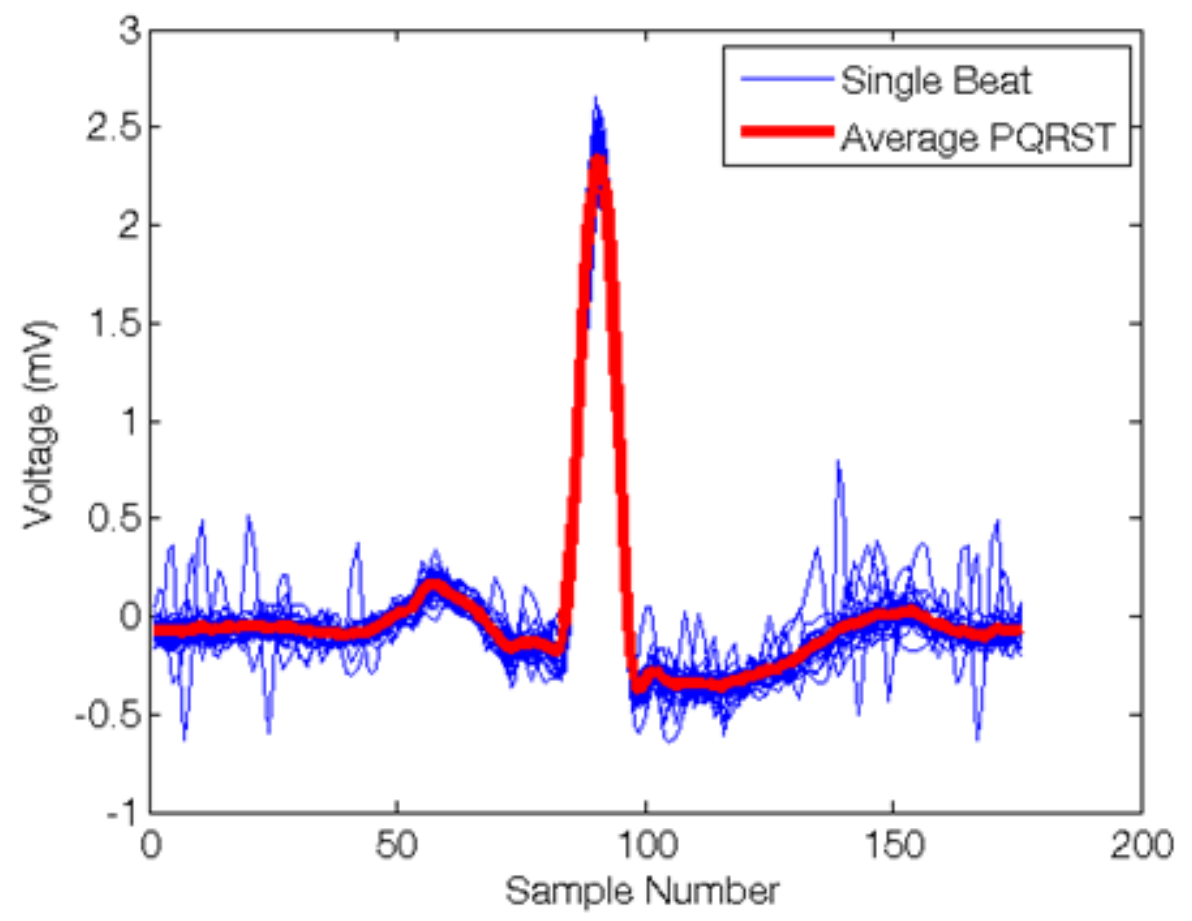

(b)

Figure 4.2 Overlaying all beats in a 30 second analysis window to determine the ensemble averaged PQRST template for (a) clean ECG and (b) noisier ECG 


\subsubsection{Determining SQI}

Assuming the ensemble averaged PQRST template is the ideal PQRST complex for the analysis window, any difference between it and the individual PQRST complexes can be considered noise. Thus, a SNR estimate is calculated for each $W_{m}[n]$ in the analysis window as follows. First, the RMS value of $W_{a v g}[n]$ is calculated and then squared to represent the signal power of the ideal PQRST complex $\left(W_{\text {avg,rms }}\right)$. Then the noise power for each beat is approximated by calculating the RMS value of the differences between the ensemble averaged PQRST template and that individual beat (Noise $e_{m, r m s}$, for the $m^{\text {th }}$ beat), as described in (4.3). The SNR for each $W_{m}[n]$ is calculated in $\mathrm{dB}$ using the values of $W_{\text {avg,rms }}$ and Noise $e_{m, r m s}$, as indicated in (4.2).

$$
\begin{gathered}
S N R_{m}=10 \log _{10}\left(\frac{W_{\text {avg }, r m s}}{\text { Noise }_{m, r m s}}\right)^{2} \\
S N R_{m}=10 \log _{10} \frac{\frac{1}{N} \sum_{n=1}^{N} W_{\text {avg }}[n]^{2}}{\frac{1}{N} \sum_{n=1}^{N}\left(W_{m}[n]-W_{\text {avg }}[n]\right)^{2}}
\end{gathered}
$$

The result is a set of SNR estimates, $\left\{S N R_{m}\right\}$, one for each heart beat. SQIs are then derived from this set of SNR estimates. A total of four SQIs were investigated - these are defined in Table 4.2 and further elaborated upon below.

\section{Table 4.2 Various SQI Definitions}

\begin{tabular}{|c|c|}
\hline Investigated SQI & Definition \\
\hline $\mathrm{SQI}_{\text {min }}$ & Minimum SNR in $\left\{S N R_{m}\right\}$ \\
\hline $\mathrm{SQI}_{25}$ & $25^{\text {th }}$ percentile SNR in $\left\{S N R_{m}\right\}$ \\
\hline $\mathrm{SQI}_{\text {mean }}$ & Mean SNR in $\left\{S N R_{m}\right\}$ \\
\hline $\mathrm{SQI}_{\text {median }}$ & Median SNR in $\left\{S N R_{m}\right\}$ \\
\hline
\end{tabular}


For each 30 second segment $S Q I_{\text {min }}, S Q I_{25}, S Q I_{\text {mean }}$, and $S Q I_{\text {median }}$ were calculated following their definitions listed in Table 4.2. $S Q I_{\min }$ is simply the minimum $S N R_{m}$ in the analysis window's set of SNRs, as shown in (4.4)

$$
S Q I_{\min }=\min _{m}\left\{S N R_{m}\right\}
$$

$S N R_{25}$ is the $25^{\text {th }}$ percentile SNR found within $\left\{S N R_{m}\right\}$. The $25^{\text {th }}$ percentile SNR is calculated using MATLAB's 'prctile' function, which calculates percentiles in the following manner. First $\left\{S N R_{m}\right\}$ is ordered from least to greatest. Then each $S N R_{m}$ is given a percentile ranking, $k$, according to (4.5),

$$
\text { Percentile }_{k}=S N R_{m}, \text { where } k=100 \times \frac{m-0.5}{M}
$$

where $m$ is the index of the ordered set $\left\{S N R_{m}\right\}$ and $M$ is the total number of heart beats in the 30 second segment. If no $S N R_{m}$ in the set $\left\{S N R_{m}\right\}$ is identified as the $25^{\text {th }}$ percentile (i.e., $k=25$ ), the then $25^{\text {th }}$ percentile value is linearly interpolated from the next highest and lowest percentiles contained within $\left\{S N R_{m}\right\}$.

$S Q I_{\text {mean }}$ is calculated by taking the average of $\left\{S N R_{m}\right\}$ as shown in (4.6).

$$
S Q I=\frac{1}{M} \sum_{m=1}^{M} S N R_{m}
$$

Lastly, $S Q I_{\text {median }}$ is the median SNR (i.e., the $50^{\text {th }}$ percentile) in the set $\left\{S N R_{m}\right\}$, as described in (4.5) except with $k=50$. 


\subsection{Validation Methods}

To test performance of these SQIs, ECG was artificially contaminated with known amounts of noise to get calibrated signals. SQIs were then generated for these calibrated signals and compared back to the known noise levels used to create the calibrated signals. Calibrated signals were created using ECG records from Physionet's Long-Term ST Database [38], [39] and motion artifact from Physionet's MIT-BIH Noise Stress Test Database [74]. The SQI algorithm was assessed using both non-ischemic and ischemic ECG data to ensure consistent performance regardless of ST segment deviations.

\subsubsection{ECG Signal Data}

As explained above, validation of the SQI algorithm was performed using ECG data from Physionet's Long-Term ST Database. These records are sampled at a rate of $250 \mathrm{~Hz}$, as mentioned in Section 4.1.1.

The target application of the SQI algorithm is to reduce false myocardial ischemia alarms using the ECG signal quality. Since the target data are going to come from potentially ischemic patients, it is necessary to validate the performance of the SQI algorithm using both healthy ECG and ECG containing ST deviations. Recall, the SQIs are based on the assumption that the ensemble averaged PQRST template reflects the ideal PQRST complex for the entire 30 second analysis window. Given that the ST segment deviates during an ischemic episode, it is necessary to validate that this deviation does not affect SQI performance.

To accomplish this, the 's20031' record was chosen, which contains two unspecified ECG leads, herein referred to as 'channel 1' and 'channel 2'. The 's20031' record was 
chosen because it contained an ischemic episode lasting approximately 30 minutes in length followed by a 30 minute period of non-ischemic ECG data. The 30 minute length was chosen to match the length of the 'em' motion artifact record from the MIT-BIH Noise Stress Test Database. The ischemic episode that was used to validate the SQI algorithm began at an elapsed time of 31 minutes and 13 seconds and lasted until 1 hour, 6 minutes and 55 seconds. The ischemic episode is summarized in Table 4.3.

\section{Table 4.3 Begining, end, and maximal deviation measurements and their elapsed times times for a 30 minute iscehmic episode in record '20031'}

\begin{tabular}{|c|c|c|c|c|}
\hline & & Start & Maximal Deviation & End \\
\hline \multirow{2}{*}{$\begin{array}{l}= \\
\mathbb{\Xi} \\
\text { J्ञ } \\
\text { J }\end{array}$} & $\begin{array}{l}\text { Elapsed Time } \\
\text { (hh:mm:ss) }\end{array}$ & $00: 32: 03$ & $00: 43: 03$ & 01:04:57 \\
\hline & ST Deviation & $0.055 \mathrm{mV}$ & $-0.322 \mathrm{mV}$ & $-0.049 \mathrm{mV}$ \\
\hline \multirow{2}{*}{ 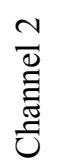 } & $\begin{array}{c}\text { Elapsed Time } \\
\text { (hh:mm:ss) }\end{array}$ & $00: 31: 13$ & $00: 44: 41$ & 01:06:55 \\
\hline & ST Deviation & $0.052 \mathrm{mV}$ & $-0.461 \mathrm{mV}$ & $-0.049 \mathrm{mV}$ \\
\hline
\end{tabular}

Thus, ischemic ECG data was taken from between the elapsed times of 30 to 60 minutes, and non-ischemic data was taken from between the elapsed times of $85-115$ minutes. Both of these 30 minute periods of ECG data were deemed to be contaminant free by visual inspection. Visual inspection also confirmed that the 30 minutes of healthy ECG contained no ST deviations.

\subsubsection{Calibrating Test Signals}

The noise used to create the calibrated signals for both the non-ischemic and ischemic records was motion artifact taken from the 'em' record in the MIT-BIH Noise Stress Test 
Database. This motion artifact was captured for Physionet by placing electrodes on the limbs of a physically active subject, such that the subject's ECG was not visible. Motion artifact was then recorded as the subject engaged in physical activity [74]. The 'em' record is sampled at a rate of $360 \mathrm{~Hz}$, thus it had to be down-sampled down to $250 \mathrm{~Hz}$ before it was scaled and added to the Physionet ECG records to make the ECGs with calibrated SNR. This down-sampling was performed using MATLAB's 'resample' function, which employs an anti-aliasing filter during the down-sampling.

The SQI algorithm is based on 30 second analysis windows. Thus, to appropriately calibrate the noise levels, the 30 minutes of motion artifact and of ECG were first divided into 30 second non-overlapping segments. The resulting 60 segments of motion artifact were individually scaled at five levels and added to the 60 segments of ECG to provide five copies of each ECG segment, each with a different SNR evenly distributed between $-10 \mathrm{~dB}$ and $10 \mathrm{~dB}$ in steps of 5 . This provided a total number of 300 segments for each ECG channel. This procedure was carried out for each of the following:

- 30 minutes of 's20031' - no ischemic episode

- 30 minutes of 's20031' - with ischemic episode

To achieve the calibrated SNR levels just mentioned, the signal and noise powers were calculated as described below.

\subsubsection{Establishing ECG Signal Power}

Following the guidelines set out in the MIT-BIH Noise Stress Test Database [74], ECG signal power was calculated individually for each 30 second segment by first measuring the peak-to-peak amplitude of each QRS complex in that segment. The largest and 
smallest $5 \%$ of measurements were discarded and the average peak-to-peak amplitude,

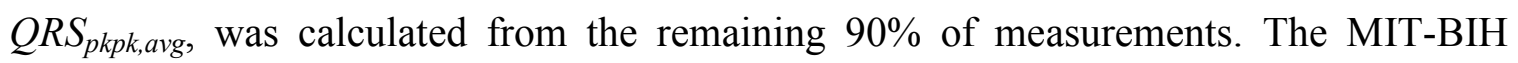
Noise Stress Test Database recommends calculating ECG signal power by ignoring the $\mathrm{P}$ and T waves while treating the QRS complex as one quarter of a pure sine wave [74]. Consequently, the ECG signal power for each segment was calculated by squaring the average peak-to-peak QRS amplitude and dividing by a factor of 8, as described in (4.7)

$$
P_{E C G}=\frac{\left(Q R S_{p k p k, a v g}\right)^{2}}{8}
$$

where $P_{E C G}$ is the ECG signal power in the 30 second segment.

\subsubsection{Establishing Motion Artifact Noise Power}

Similarly, also following the guidelines set out in the MIT-BIH Noise Stress Test Database [74], the noise power for each 30 second motion artifact segment was calculated as follows. Each 30 second segment was divided into 1 second intervals (Noise $i[n]$, where $i$ is used to represent the $i^{t h}$ second). For the down-sampled rate of 250 $\mathrm{Hz}$, this corresponds to $N=250$ samples. The average baseline, $B_{i}$, was calculated for each 1 second interval, as in (4.8).

$$
B_{i}=\frac{1}{N} \sum_{n=1}^{N} \operatorname{Noise}_{i}[n]
$$

The RMS of the differences between $B_{i}$ and the motion artifact amplitude was calculated for each interval, as shown in (4.9). 


$$
R M S_{i}=\sqrt{\frac{1}{N} \sum_{n=1}^{N}\left(\text { Noise }_{i}[n]-B_{i}\right)^{2}}
$$

where $R M S_{i}$ is the RMS value of the differences between $B_{i}$ and the motion artifact amplitude for the $i^{\text {th }}$ interval. The smallest 5\% and largest 5\%RMS values are discarded and the segment's average RMS value, Noise $e_{a v g, R M S}$, is calculated from the remaining $90 \%$ of values. The noise signal power is then calculated by squaring Noise $e_{\text {avg,RMS }}$, as in

$$
P_{\text {Noise }}=\text { Noise } e_{\text {avg }, R M S}^{2}
$$

where $P_{\text {Noise }}$ is the signal power of the noise in the 30 second segment.

\subsubsection{Scaling Noise to Calibrate SNR}

Five scaling factors, $C_{k}$, are then calculated for the motion artifact such that the segment's

SNR is calibrated to $-10,-5,0,5$, and $10 \mathrm{~dB}$, as shown in (4.11) and expanded in (4.12).

$$
\begin{gathered}
\{-10,-5,0,5,10\}=10 \log _{10}\left(\frac{P_{E C G}}{C_{k} \times P_{\text {Noise }}}\right) \\
C_{k}=\frac{P_{E C G}}{10^{\frac{\{-10,-5,0,5,10\}}{10}} \times P_{\text {Noise }}}
\end{gathered}
$$

Figure 4.3 demonstrates the same 18 seconds of ECG data that has been contaminated with motion artifact scaled by five $C_{k}$ scaling factors, note the differing y-axis scales between plots. 
SNR $10 \mathrm{~dB}$

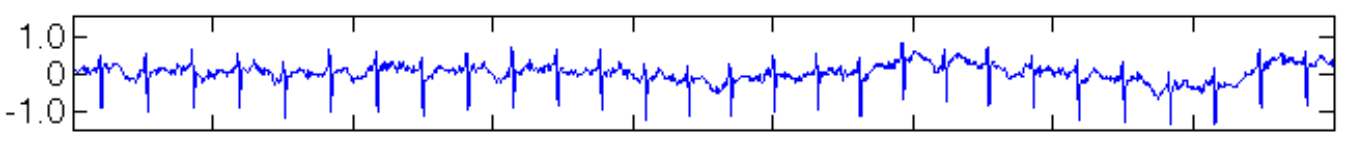

SNR $5 \mathrm{~dB}$

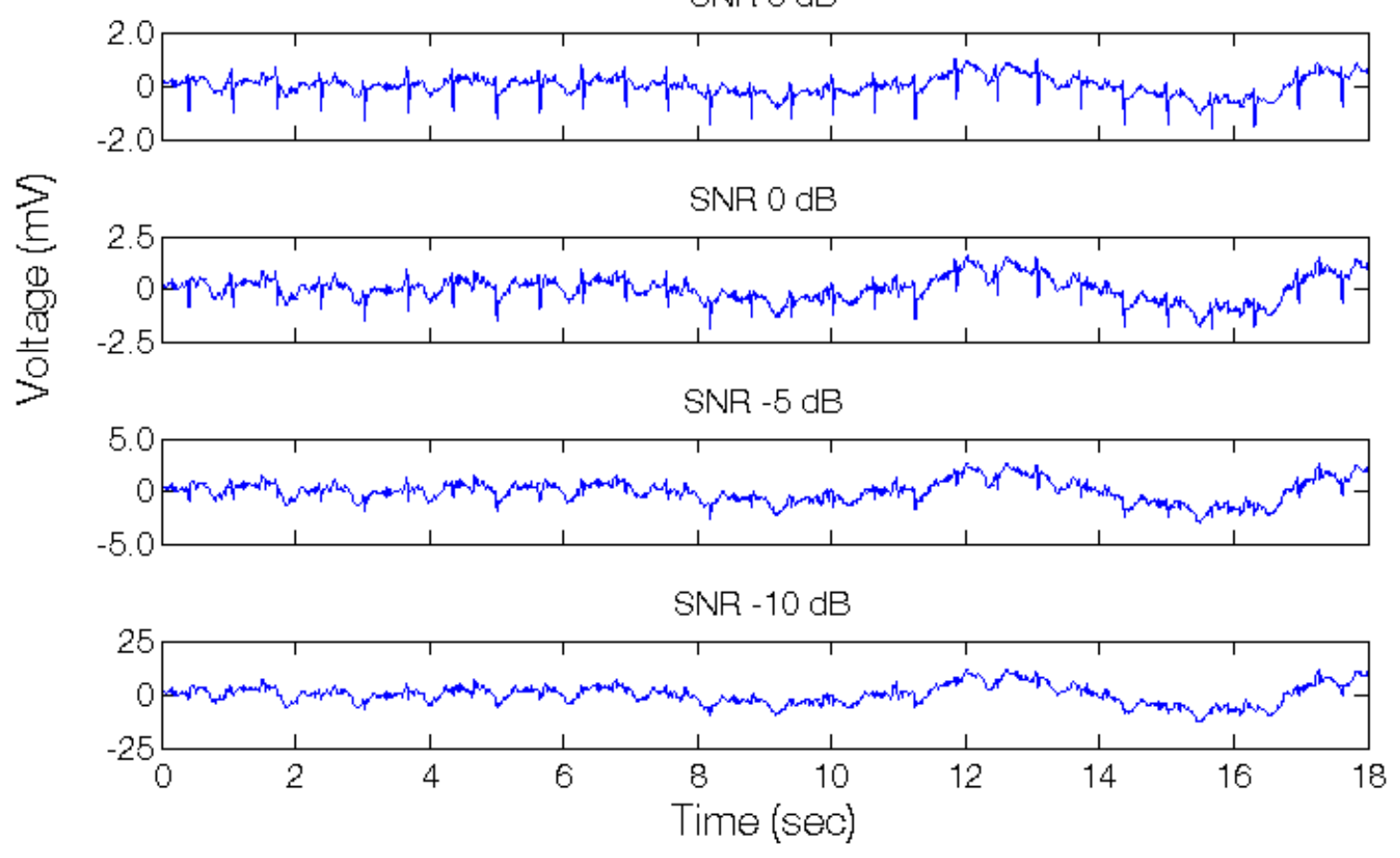

Figure 4.3 The same ECG data with five levels of calibrated motion artifact noise

This calibration procedure was repeated for each 30 second segment, such that each segment was individually calibrated to have the appropriate level of SNR.

\subsubsection{Generating SQIs}

\subsubsection{Locating and Aligning R Peaks in the Physionet Data}

To actually generate SQIs for the Physionet ECG records, it was necessary to first identify the location of all R peaks to ensure proper alignment of the PQRST complexes when ensemble averaging. While many methods exist to identify QRS complexes, as discussed in Section 4.1.3, in some cases these methods can misidentify R peak location, 
simply miss a QRS complex all together, or falsely detect an R peak when there actually is no beat. To avoid this potentially confounding factor, manually corrected beat annotations provided by Physionet (named, 'atr') were used to identify the location of all $R$ peaks in the ECG data.

It was noticed, however, that there is a tolerance on the location of the $\mathrm{R}$ peak relative to the indicated 'atr' sample number; this could introduce a delay when ensemble averaging, resulting in a potentially biased SQI. As a result, the Physionet annotations were used to approximately identify R peak locations, then cross-correlation was performed between the identified PQRST complex and the growing ensemble averaged PQRST template. This cross correlation was used to determine any potential delay between the identified complex and the template, and then to correct that delay such that the incoming R peak was exactly aligned with the template's $\mathrm{R}$ peak. $\mathrm{R}$ peaks were only shifted by a maximum of 7 samples based on the cross-correlation alignment. This limit prevents the potential for accidental alignment of the $\mathrm{R}$ peak with adjacent $\mathrm{P}$ or $\mathrm{T}$ waves.

\subsubsection{SQI Estimates}

Four SQI metrics were defined in Section 4.1.6. Each of the four SQI metrics were generated for every calibrated 30 second segment described in Section 4.2.2.

\subsubsection{Determining Feasibility of Real-Time Deployment}

The SQI is intended for real-time applications. To determine feasibility of SQI deployment in real-time, 600 SQIs were generated and the computation time required by each SQI was recorded using MATLAB's 'tic' and 'toc' commands. MATLAB's 'tic'

command sets a stopwatch timer within the MATLAB environment while the 'toc' 
command is used to read the elapsed stopwatch time. Before the generation of each of the $600 \mathrm{SQIs}$, the 'tic' command was issued to initiate the stopwatch, then following the generation the SQI, the elapsed time was recorded via the 'toc' command.

This test was carried out on a PC equipped with an Intel Core i5-470UM CPU, which has two cores running at $1.33 \mathrm{GHz}$. On board memory was $4 \mathrm{~GB}, 1066 \mathrm{MHz}$ DDR3. The mean computation time across each of the 600 SQIs was found to be 0.05 seconds with a standard deviation of 0.06 seconds.

Thus, on average, it takes 0.05 seconds to generate an SQI that represents 30 seconds of ECG data. Since SQIs are generated every 5 seconds, the computation time required for single SQI means that real-time SQI generation is feasible. Several factors will influence total computation time upon real-time implementation. The addition of QRS detection, which was not implemented in this thesis (as discussed in Section 4.2.3.1) will result in increased computation time; however, real-time QRS detection methods, such as those outline in Section 4.1.3, will not increase SQI computation time beyond the feasibility of real-time implementation.

\subsection{Results}

As discussed in Section 4.2.2, 300 segments were created for each channel of both the non-ischemic and ischemic data, resulting in a total 1,200 segments. Four SQIs were then generated for each of these 1,200 segments. Figure 4.4 shows the relationship between these SQIs and the known SNR levels of their respective segments for ECG data without ST deviations, while Figure 4.5 shows a similar relationship when applied to ECG data containing ST deviations. 


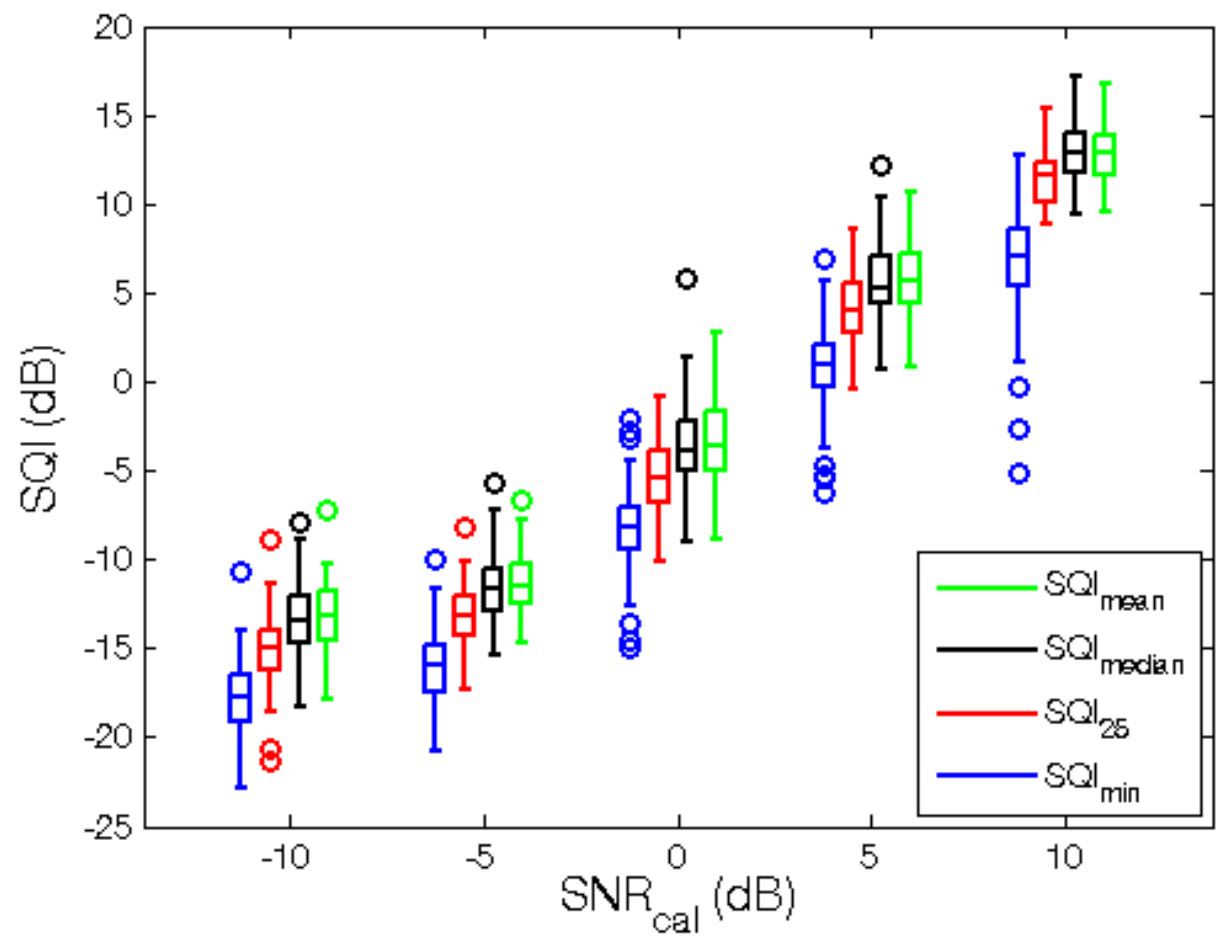

Figure 4.4 Boxplot showing the relationship between calibrated levels of SNR and four SQI metrics for ECG data without ST deviations

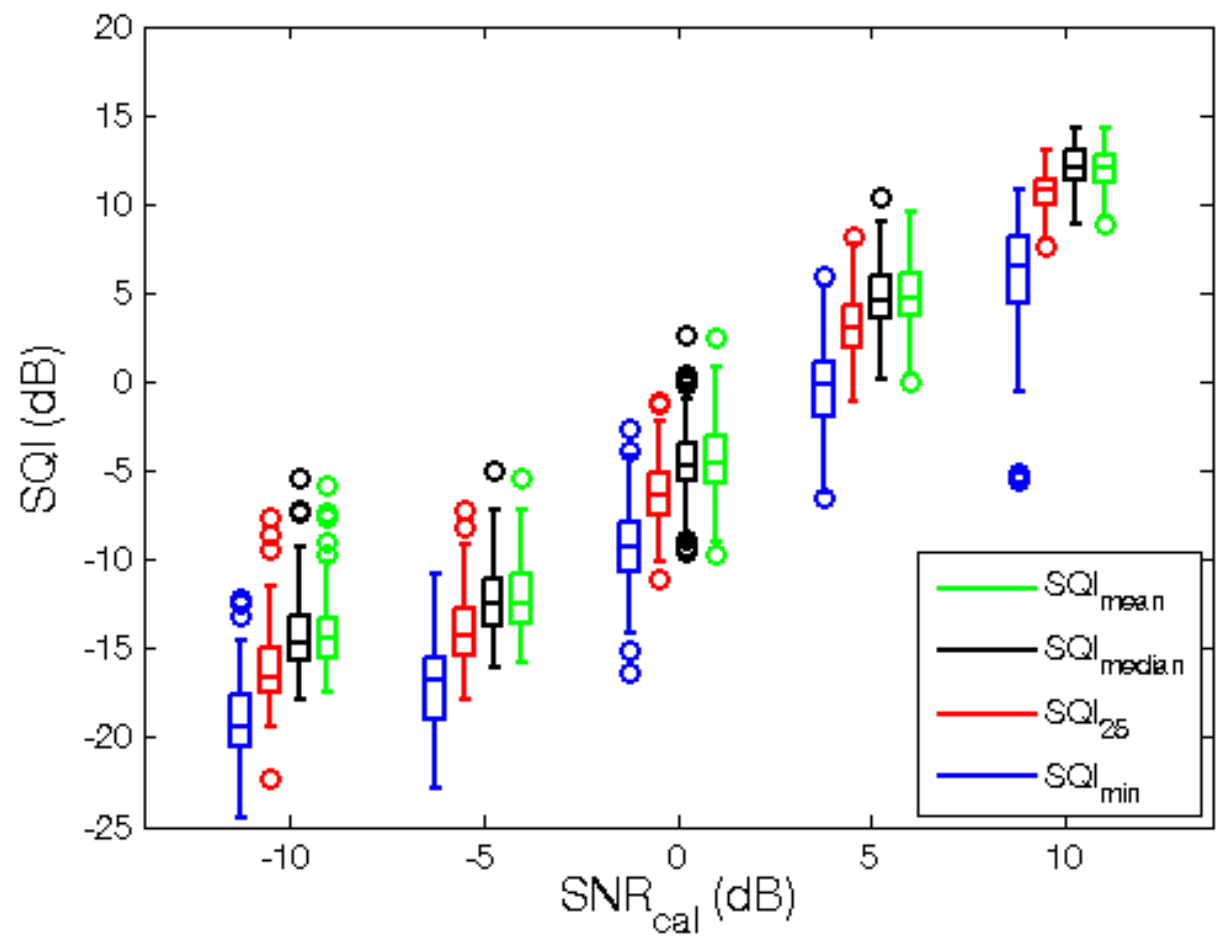

Figure 4.5 Boxplot showing the relationship between calibrated levels of SNR and four SQI metrics for ECG data with ST deviations 
A Pearson correlation coefficient was calculated between the SQIs and the calibrated SNRs for each of the cases shown in Table 4.4.

Table 4.4 Correlation coefficients for the validation of four metrics used in the SQI algorithm for both non-ischemic and ischemic data

\begin{tabular}{|c|c|c|c|}
\hline & SQI Metric & Channel 1 & Channel 2 \\
\hline \multirow{4}{*}{ 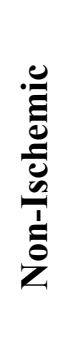 } & $\mathrm{SQI}_{\min }$ & 0.9501 & 0.8720 \\
\hline & $\mathrm{SQI}_{25}$ & 0.9647 & 0.8814 \\
\hline & $\mathrm{SQI}_{\text {median }}$ & 0.9616 & 0.8832 \\
\hline & $\mathrm{SQI} \mathrm{I}_{\text {mean }}$ & 0.9632 & 0.8837 \\
\hline \multirow{4}{*}{ 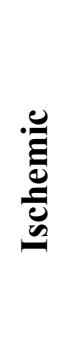 } & $\mathrm{SQI}_{\min }$ & 0.9412 & 0.8513 \\
\hline & $\mathrm{SQI}_{25}$ & 0.9625 & 0.8572 \\
\hline & $\mathrm{SQI} \mathrm{I}_{\text {median }}$ & 0.9604 & 0.8556 \\
\hline & $\mathrm{SQI}_{\text {mean }}$ & 0.9610 & 0.8595 \\
\hline
\end{tabular}

\subsection{Discussion}

This thesis work seeks to establish that the proposed SQI reflects ECG signal quality. The absolute value of the SQI is of little importance; what matters is being able to differentiate between periods of poor and good signal quality (i.e., the SQI value for poor quality ECG relative to the SQI value for good quality ECG signals). Correlation is a measure of the linear relationship between two variables, and thus is an appropriate measure of SQI performance. In all four cases, strong correlation (Pearson correlation coefficient $>0.85$ ) was observed between the calibrated SNR of a segment and its 
affiliated SQI. As described in Section 4.2.2, calibrating the SNR for each segment was essentially accomplished by averaging the motion artifact amplitude over 30 second segments. Thus, within those 30 seconds, there will be periods with more severe artifact than others. This is especially visible in the lower correlation coefficients when contaminating with 'channel 2' of the motion artifact.

Outliers showing the SQI to have overestimated the SNR were investigated and found to be due to periodicity of the motion artifact matching the HR within the segment it contaminated. Recall, the motion artifact was captured from physically active volunteers; it is possible that at times the volunteer was involved in repetitive motion. Figure 4.6 shows an outlying 30 second segment calibrated to have a $-10 \mathrm{~dB}$ SNR that returned an SQI of -5.1 . The segment immediately before it was found to have an SQI of -19.2 and can be seen in Figure 4.7. 


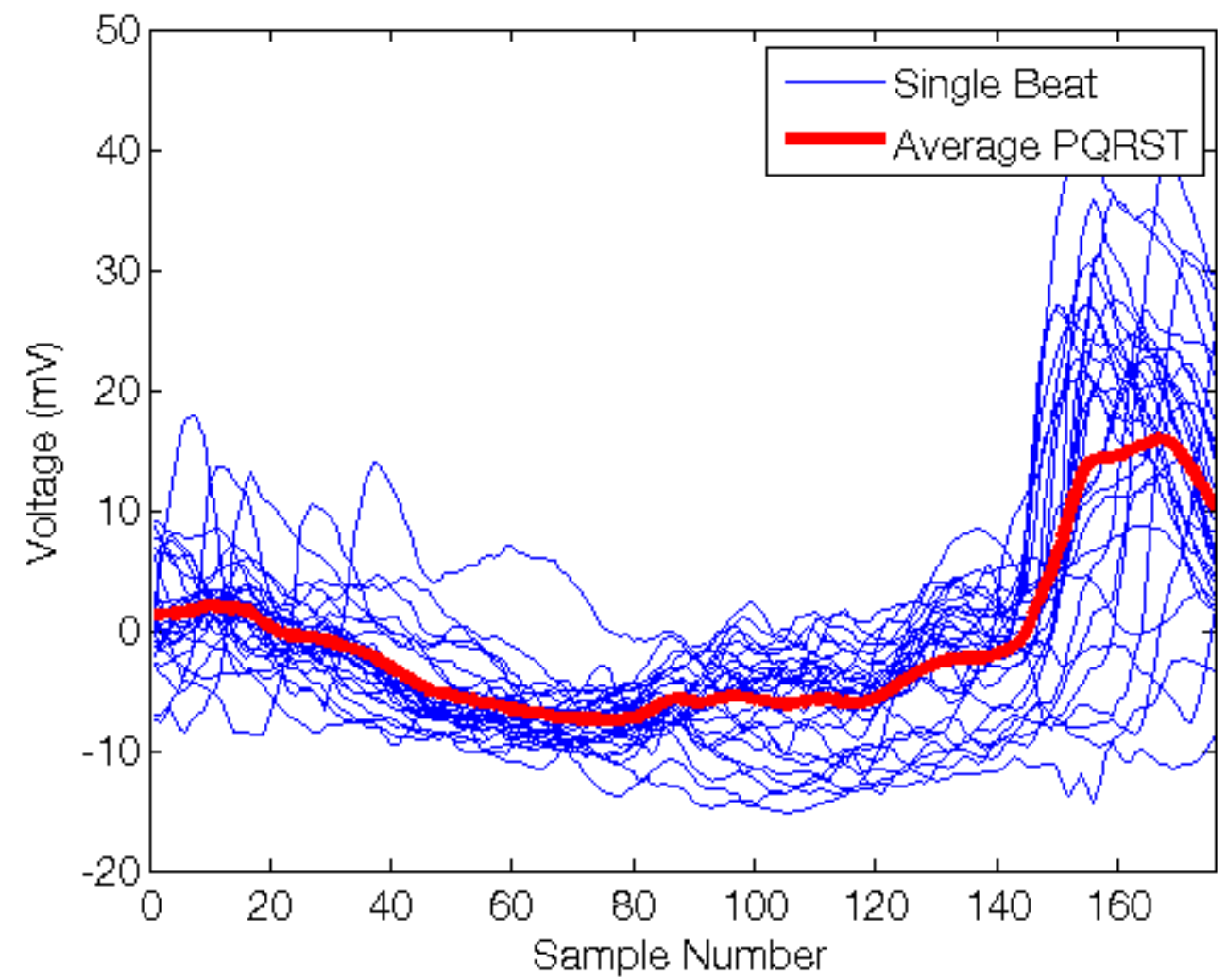

Figure 4.6 Ensemble averaging 30 seconds of ECG with periodic motion artifact that matches the HR in a segment calibrated to have a-10 dB SNR

Conversely, outliers that show that the SQI underestimated the SNR are influenced by the averaging nature of the signal power calculations described in Section 4.2.2; calibrating the SNR for each segment was essentially accomplished by averaging the motion artifact amplitude over 30 second segments. Thus, within those 30 seconds, there will be periods with more severe artifact than others. Those periods of extra poor signal quality will reduce the SQI especially if the SQI is based on the minimum SNR. 


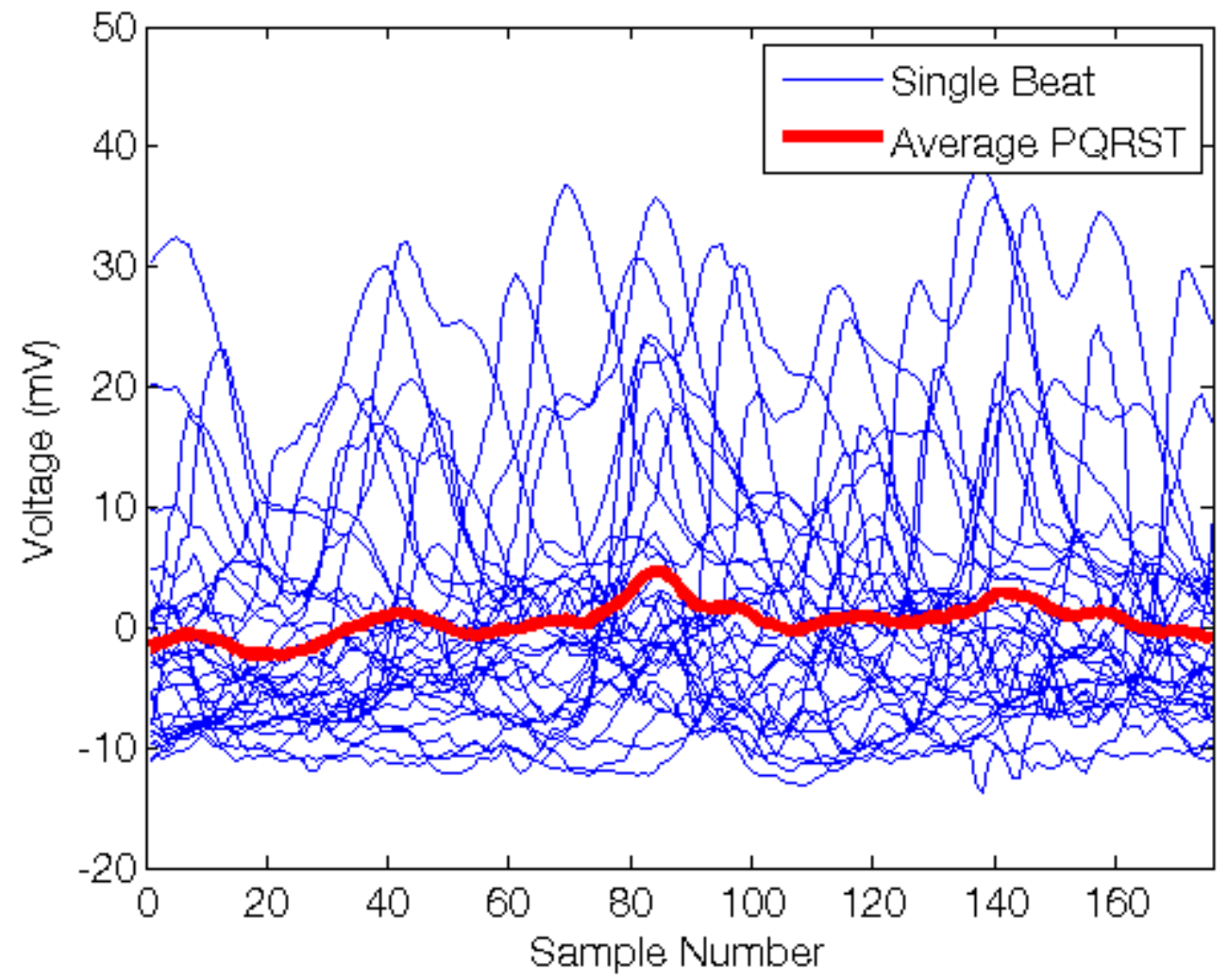

Figure 4.7 Ensemble averaging the 30 second segment immediately before the one shown in Figure 4.6

It was initially thought that the $S Q I_{\min }$ would provide the most ideally suited indicator of signal quality. This is because the goal was to produce a conservative estimate of the signal quality such that the SQVS could aggressively curb false alarms; however, it was found that this artificially decreased the estimated signal quality in the presence of a misidentified $\mathrm{R}$ peak or a premature ventricular contraction (PVC), as illustrated in Figure 4.8.

The different morphology of a PVC or the misalignment of an $\mathrm{R}$ peak can cause a significant difference to remain after subtracting the ensemble averaged PQRST 
template, $W_{\text {avg }}[n]$, thereby decreasing that beat's SNR and artificially decreasing the SQI for the whole window if using $S Q I_{\text {min }}$.

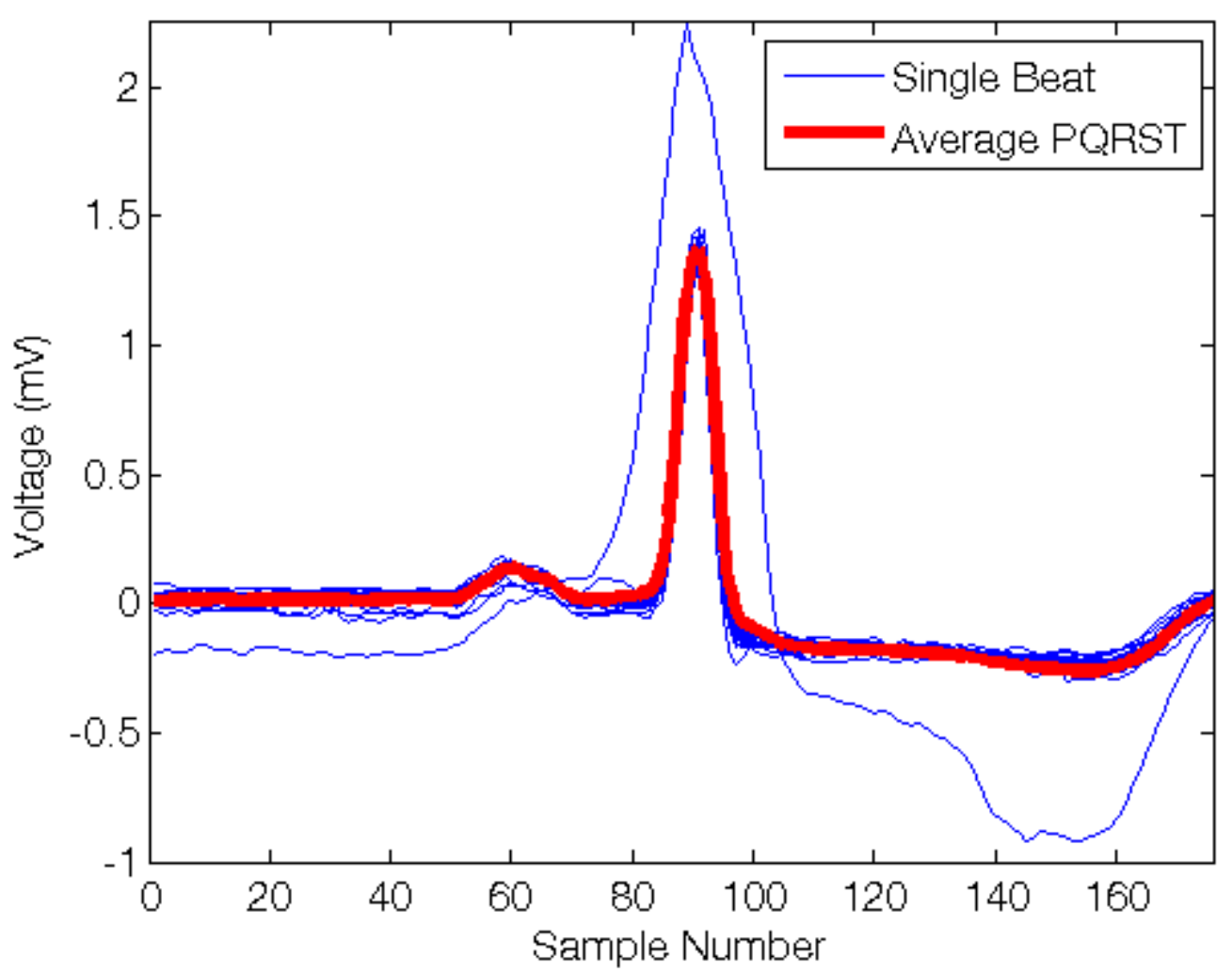

Figure 4.8 Ensemble averaging a contaminant-free 30 second segment containing a PVC

The similar correlations shown in Figure 4.4 and Figure 4.5 indicate that presence of varying ST deviations does not have a noticeable effect on the SQI algorithm. The gradual drift of the ST segment affiliated with myocardial ischemia does not result in individual PQRST complexes differing greatly from the continually updated ensemble averaged template.

Therefore, this validation using ischemic ECG data containing ST deviations indicates that the SQI algorithm is not adversely affected by ischemic ECG data. Thus, the SQI 
algorithm continues to generate SQIs that accurately reflect ECG signal quality, regardless of ST deviations.

$S Q I_{25}, S Q I_{\text {mean }}$, and $S Q I_{\text {median }}$ all exhibited very similar correlation with the actual level of SNR (refer to Table 4.4). $S Q I_{25}$ consistently exhibiting the highest correlation for Channel 1 data. Consequently, $S Q I_{25}$ was chosen for further investigation because it provides a conservative estimate of the signal quality, while still allowing from the occurrence of ECG morphologies such as PVCs, as well as misaligned R peaks.

\subsection{Summary}

SQIs that reflect the actual biosignal quality of ECG data (both with and without ST deviations) can be generated by comparing each PQRST waveform in a 30 second segment to an ensemble averaged PQRST template. $S Q I_{25}$, the $25^{\text {th }}$ percentile SNR found within that 30 second segment, provides the most desirable representation of signal quality for the SQVS application. This is because it affords flexibility to account for other PQRST morphologies (such as PVCs) and misidentified R peaks but still provides a conservative estimate of the signal quality. This helps to aggressively gate false myocardial ischemia alarms. 


\section{Investigating ECG Signal Quality and False Alarms}

Before investigating the effect of SQVS-gating alarms it was necessary to establish that increased noise in an ECG record (e.g., motion artifact) did, in fact, result in false alarms. Thus, the effect of motion artifact on ST deviation estimates was investigated in conjunction with its relationship to the SQI. Outcomes of this investigation spoke to the feasibility of gating alarms via the SQVS.

To test this, a control signal was played through the bedside monitor and the resulting ST values were analyzed to confirm that no alarms were generated by the alarm generating FSM described in Section 3.5. Then the control signal was contaminated with motion artifact to get the test signal, as described below in Section 5.1. This test signal was then played through the bedside monitor and the resulting ST values were analyzed for false alarms. In both cases, SQIs were simultaneously generated following the description in Chapter 4 to assess the signal quality of the control and test signals.

\subsection{Establishing Control and Test Signals}

Control and test signals used to investigate the effect of signal quality on false alarms were established using 30 minutes of ECG data from the 's20011' record in Physionet's Long-Term ST Database [39]. These 30 minutes were selected such that there were no notable ST deviations. Additionally, the signal quality was verified by visual inspection to ensure the recording had little to no contaminants. The 's20011' record was selected because it contains Lead II and V2 ECG data. These unaltered 30 minutes of ECG were taken as the control signal. 
Motion artifact from the 'em' record in the MIT-BIH Noise Stress Test Database [74] was used to contaminate the control signal in order to create the test signal. Two channels of motion artifact were used to create the test signal - channel 1 of 'em' was added to the Lead II signal and channel 2 of 'em' was added to the V2 signal. Motion artifact was down-sampled from $360 \mathrm{~Hz}$ to $250 \mathrm{~Hz}$ in order to match the sampling rate of the ECG data prior to its addition. This down-sampling was performed using MATLAB's 'resample' function, which employs an anti-aliasing filter during the down-sampling. Motion artifact was directly added to the ECG, without any scaling, to create alternating 5 minute periods of clean and contaminated ECG (e.g., 5 minutes of clean ECG, 5 minutes of contaminated ECG, 5 minutes of clean ECG..., etc.). The transition between clean and contaminated ECG is visible in Figure 5.1. 


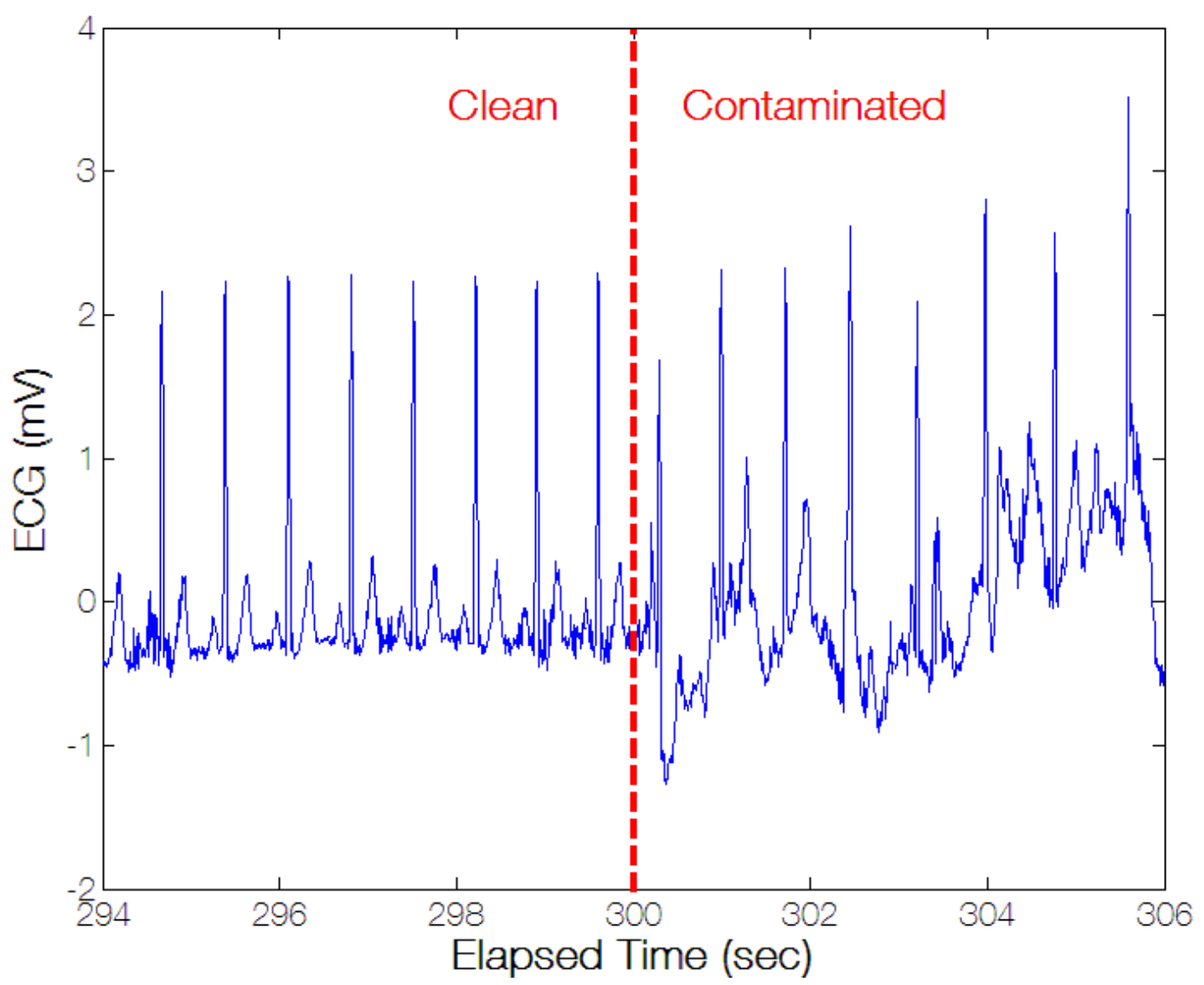

Figure 5.1 Transition from clean to contaminated ECG in the test signal used to investigate the relationship between ECG signal quality and false alarms

To characterize the components of these control and test signals, RMS values of the noise and ECG were calculated to estimate the signal power of each lead/channel. These RMS values were obtained using a 30 second sliding window that shifted in 5 seconds increments, similar to the SQI generation process. The average RMS value and standard deviation characteristics for each lead and channel are included in Table 5.1. 
Table 5.1 Power of ECG and motion artifact components used for testing the relationship between ECG signal quality and false alarms

\begin{tabular}{|c|c|c|}
\hline Signal Component & Mean (mV RMS) & Standard Deviation \\
\hline ECG Lead II & 0.4172 & 0.0295 \\
\hline ECG Lead V2 & 0.2144 & 0.0134 \\
\hline $\begin{array}{c}\text { Motion Artifact } \\
\text { Channel 1 }\end{array}$ & 0.6999 & 0.1967 \\
\hline $\begin{array}{c}\text { Motion Artifact } \\
\text { Channel 2 }\end{array}$ & 0.1926 & 0.0622 \\
\hline
\end{tabular}

\subsection{Signal Routing}

As described in Chapter 3, the ECG records were provided to the bedside monitor (91369 Ultraview SL Bedside Monitor, Spacelabs Medical, Issaquah, WA, USA) via a patient simulator (MS400 Multiparameter Simulator, CONTEC Medical Systems, Qinhuangdoa, Hebei Province, P. R. China) connected to a wireless telemetry pack (Ultraview Digital Telemetry Multiparameter Transmitter 90345, Spacelabs Medical, Issaquah, WA, USA). ST values were acquired through the bedside monitor's COM port and recorded on a PC. Separately, SQIs were generated on a PC for the ECG signal using the $S Q I_{25}$ algorithm described in Chapter 4. This process, shown in Figure 5.2, was completed for both the control and test signal; with the exception that motion artifact (shown on the left side of Figure 5.2) was not added to the control signal (as explained in the previous section). ST values were synchronized to the generated SQIs following the procedure described in Section 3.4.4. 


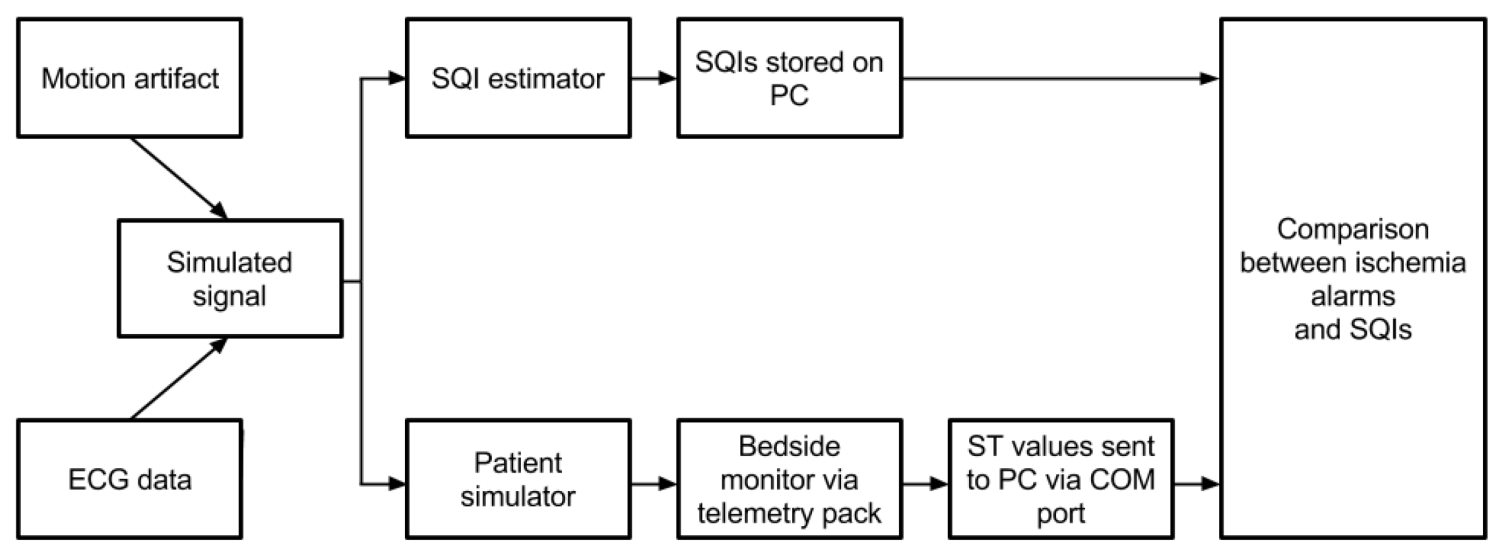

Figure 5.2 Signal flow depicting ST value generation and parallel SQI generation for testing the relationship between ECG signal quality and false alarms

\subsection{Results}

ST values for both the control and test signals were analyzed using the alarm generating FSM. Figure 5.3 and Figure 5.4, show plots of the acquired ST values and the respective SQIs for the control and test signals. Part (a) of each Figure 5.3 and Figure 5.4 show the ST values, while part (b) of each figure shows the corresponding synchronized SQIs. Red dotted lines indicate alarm condition boundaries for the ST values - for a myocardial ischemia alarm to occur, the ST value must deviate outside the alarm boundaries continuously for a period of at least one minute [5]. Myocardial ischemia alarms are indicated by hatched sections on the plots. Three alarms were triggered by Lead II of the test signal while no alarms resulted from the control signal. 


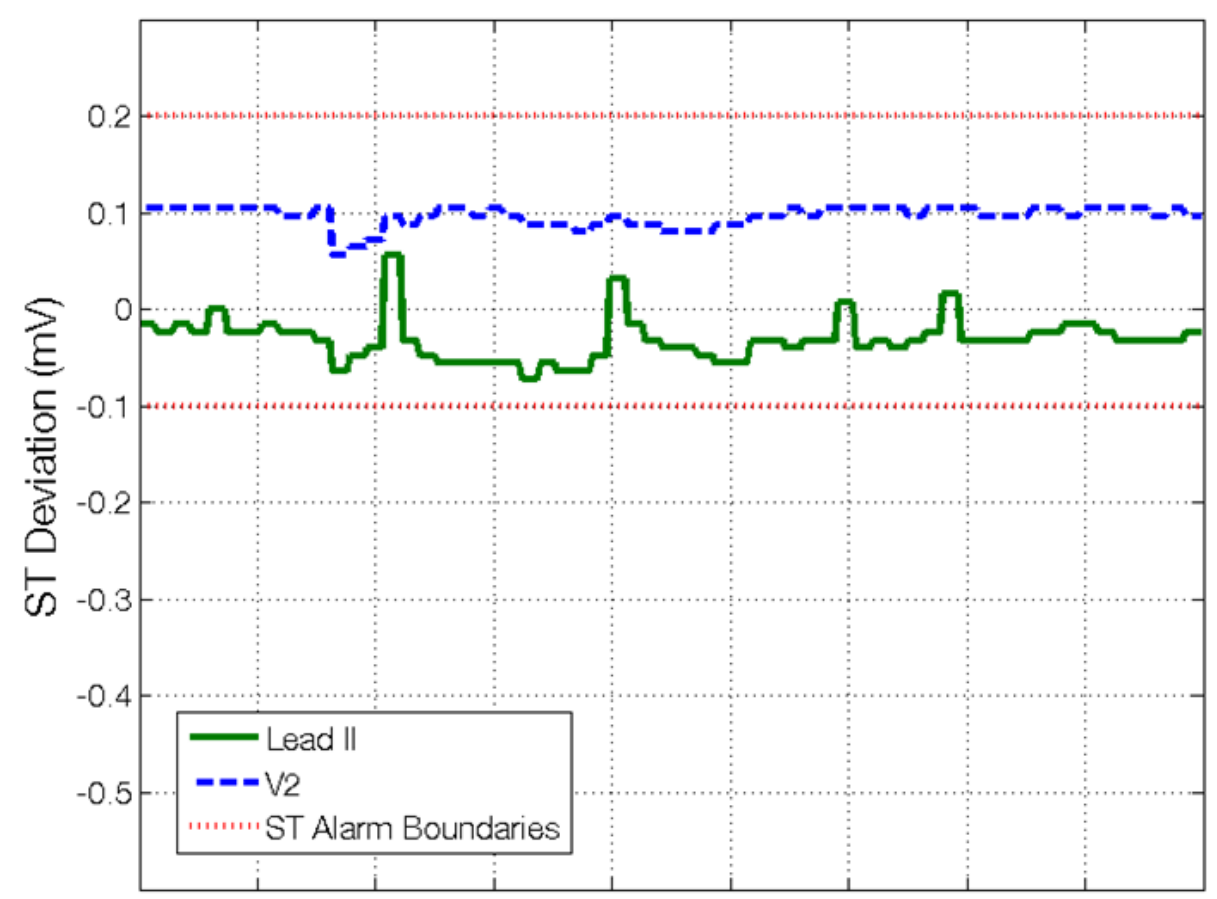

(a)

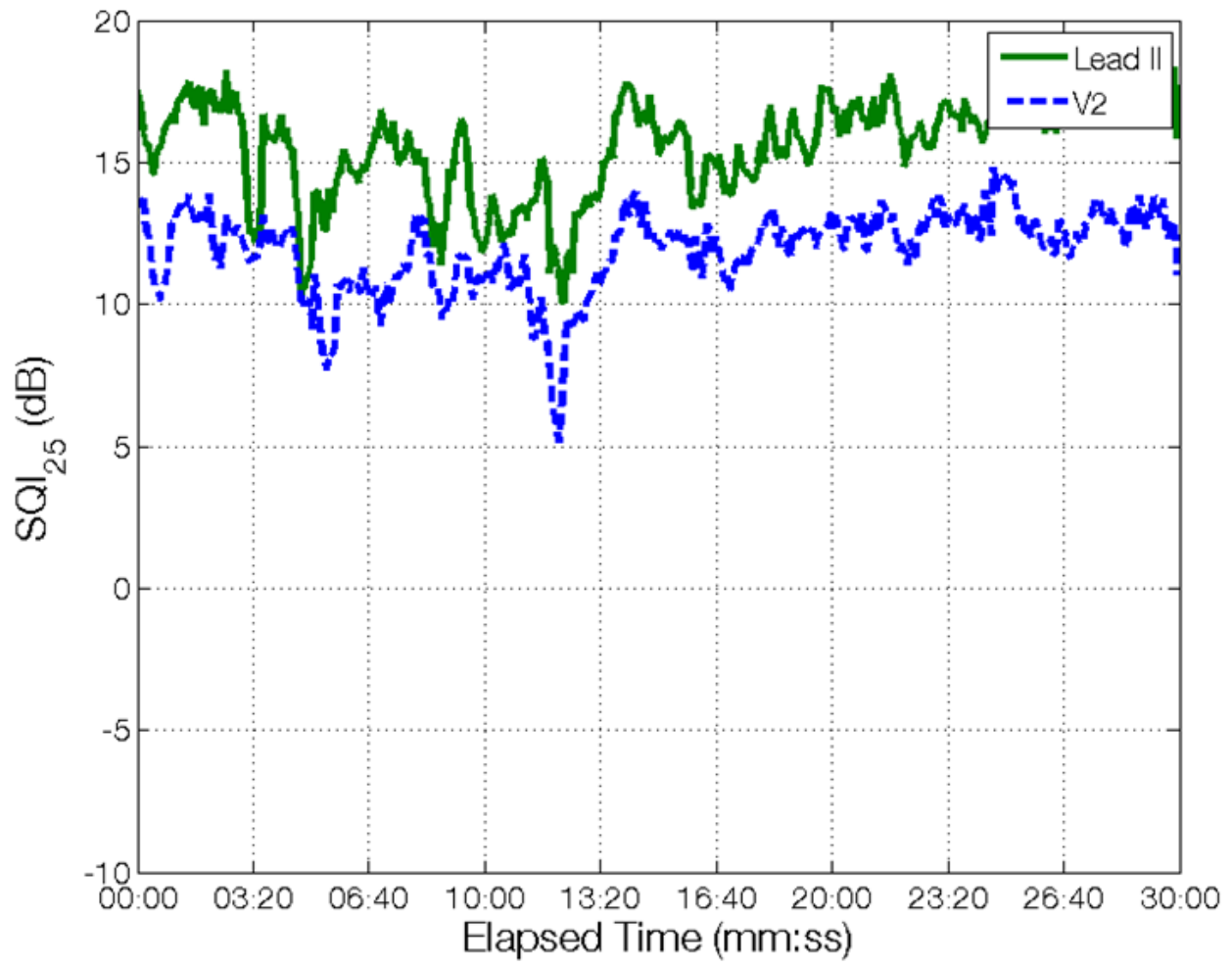

(b)

Figure 5.3 (a) ST values and (b) SQIs for the control signal. Red dotted lines indicate ischemia alarm boundaries 


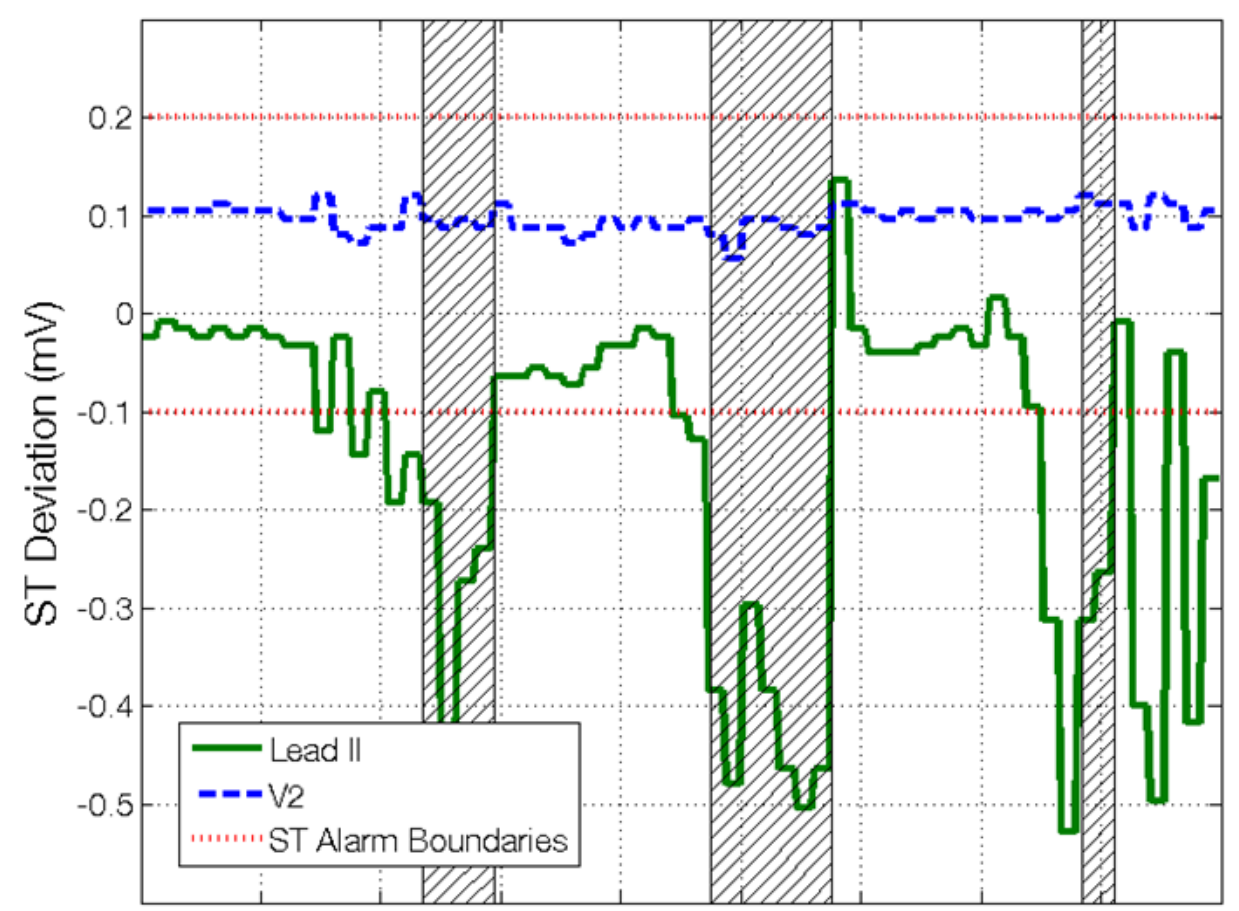

(a)

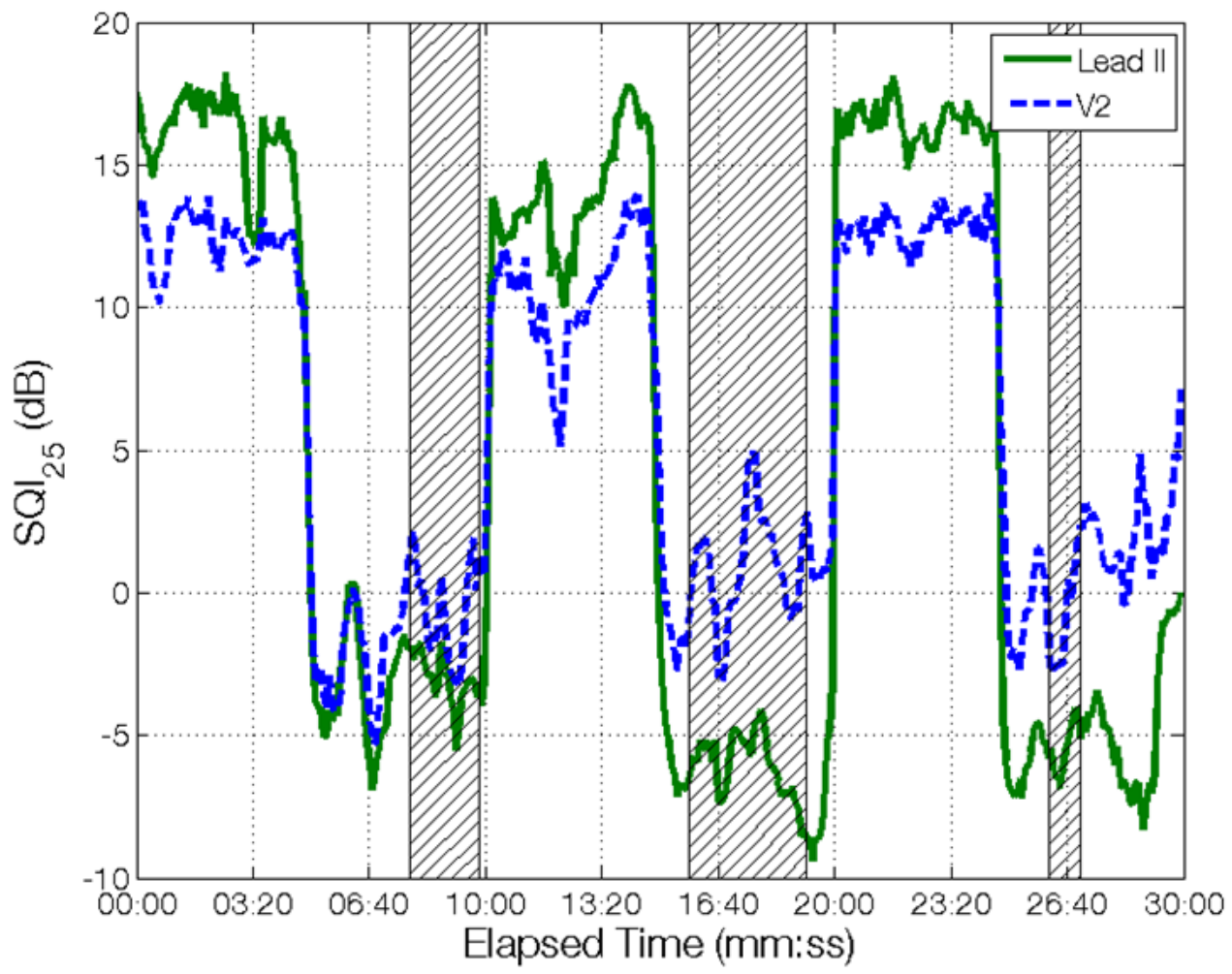

(b)

Figure 5.4 (a) ST values and (b) SQIs for the test signal. Hatched sections indicate the occurrence and duration of myocardial ischemia alarms 


\subsection{Discussion}

As expected, the ST values acquired from the control signal did not prompt any myocardial ischemia alarms from either Lead II or V2, as shown in Figure 5.3 (a). This indicated that any alarms triggered from the test signal would be false alarms resulting from the additional noise. SQIs for both leads of the control signal remained fairly steady for the signal's 30 minute duration, visible in Figure 5.3 (b).

The Lead II ST values acquired from the test signal resulted in three false positive alarms; one during each period of added motion artifact. It was noticed that contaminated sections of V2 did not result in acquisition of very different ST values from the control signal despite the 5 minute alternating periods of motion artifact being clearly visible in both leads of the test signal's SQIs (observable as large dips in part (b) of Figure 5.4). This is likely due to the relatively small signal power of both V2 and channel 2 of the 'em' motion artifact record, evidenced in the RMS values recorded in Table 5.1. Given the overall smaller signal powers, the addition of motion artifact likely had less of an impact on the bedside monitor's algorithms. The three false alarms, indicated on Figure 5.4 (a) and (b) as hatched sections, all occurred during periods with a marked decrease in SQI.

This indicates that degradation of signal quality due to motion artifact impacts the reliability of ST values estimated by the commercial bedside monitor and that unreliable ST values can trigger false myocardial ischemia positives. This also indicates that it is possible that gating myocardial ischemia alarms generated during periods of poor signal 
quality may decrease the false positive rate of the commercial detection systems without adversely affecting their sensitivity. 


\section{Signal Quality Verification System Proof of Concept}

The signal quality verification system (SQVS) proposed in this thesis consists of a biosignal quality analysis stage parallel to the existing system. The ECG biosignal quality analysis stage generates SQIs using the $S Q I_{25}$ algorithm, as described in Chapter 4. The SQIs modulate the outgoing alarms based on the quality of the ECG signal; when the SQI is low, the ECG signal quality is poor and any associated alarms would be unreliable. This chapter presents three configurations of the SQVS and evaluates their ability to improve PPV while maintaining sensitivity of myocardial ischemia detection.

\subsection{SQVS Configurations}

\subsubsection{SQVS $S_{S T}-$ Gating ST Values}

Three different configurations of the SQVS were investigated in this thesis. The first, $S Q V S_{S T}$, illustrated in Figure 6.1, uses the SQIs to gate ST deviation measurements before they reach the alarm generation stage. In this configuration, the alarm generating FSM only receives ST values when the ECG signal quality is above a set threshold as denoted by the SQIs. Recall that the FSM timers (discussed in Section 3.5.2) operate using the timestamps associated with incoming ST values. During a period of poor signal quality, no ST values reach the FSM (and thus no timestamps). Once signal quality resumes, the FSM compares the incoming ST value and timestamp to the last valid ST value. The time that ST values were gated by $S Q V S_{S T}$, is therefore counted towards whatever state was affiliated with the last valid ST value. Example operation of the $S Q V S_{S T}$ configuration is shown in Figure 6.4. In this case the FSM was put into an alarm warm up period just 
before signal quality dropped. Since the subsequent valid ST value after gating was received more than 1 minute after the last and the ST value was also beyond the alarm threshold of $-0.1 \mathrm{mV}$, an alarm was immediately triggered.

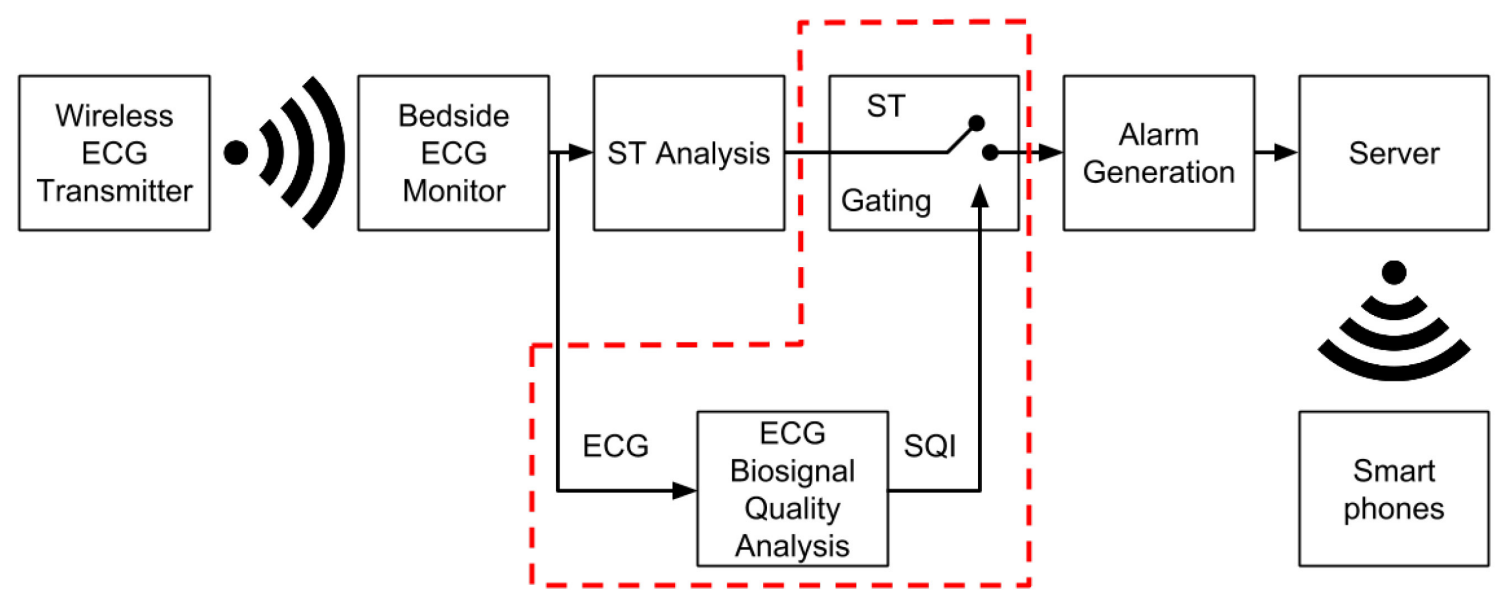

Figure 6.1 Myocardial ischemia alarm signal flow, highlight components of the $S Q V S_{S T}$ used to gate $\mathrm{ST}$ values before they reach the alarm generation stage

\subsubsection{SQVS $S_{E N}$ - Enabling /Disabling the Alarm Generation Stage}

The second, $S Q V S_{E N}$, shown in Figure 6.2, allows all filtered ST values through to the alarm generation stage, instead using the SQVS to enable or disable the alarm generating FSM depending signal quality. In this configuration, the FSM is disabled when signal quality drops. The current state and any associated timers remain unaltered until SQIs return to an acceptable level. Once signal quality is restored, the FSM resumes functioning without considering the time that elapsed while it was disabled. In this case, if the FSM entered an alarm warm up period 10 seconds before signal quality decreased, it would resume the alarm warm up state once signal quality returned to acceptable levels, still only counting the original 10 seconds toward the alarm warm up state. 


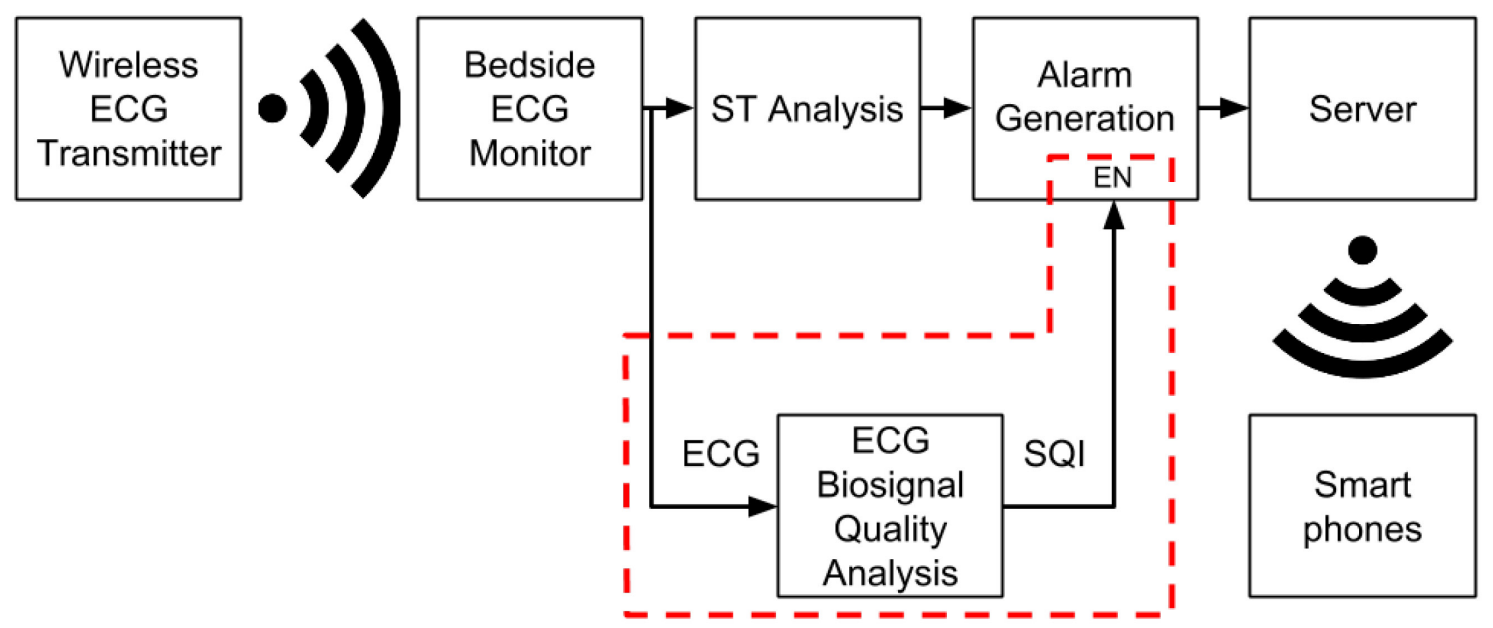

Figure 6.2 Myocardial ischemia alarm signal flow, highlighting components of the $S Q V S_{E N}$, which disables the alarm generating FSM during periods of poor signal quality

Example operation of this configuration is shown in Figure 6.4. In this case, the disabling of the FSM is shown as a blank period. When signal quality is restored, the FSM resumes operation from where it left off, effectively concatenating the periods of good signal quality. In this case, no alarm was generated because the duration of concatenated alarming ST values did not meet the required 1 minute duration.

\subsubsection{SQVS $S_{A L}$ - Gating Generated Alarms}

Lastly, $S Q V S_{A L}$ was implemented to gate the output of the alarm generation stage, as shown in Figure 6.3. In this configuration, ST values are passed through to the alarm generation stage at all times and the alarm generation stage uses all ST values to create alarms. An SQI threshold controlled signal gate is implemented after the alarms are generated. This gate stops alarms from being passed on for dissemination when the signal quality is low, as well as for the first 2 minutes after SQIs have returned to acceptable levels. 


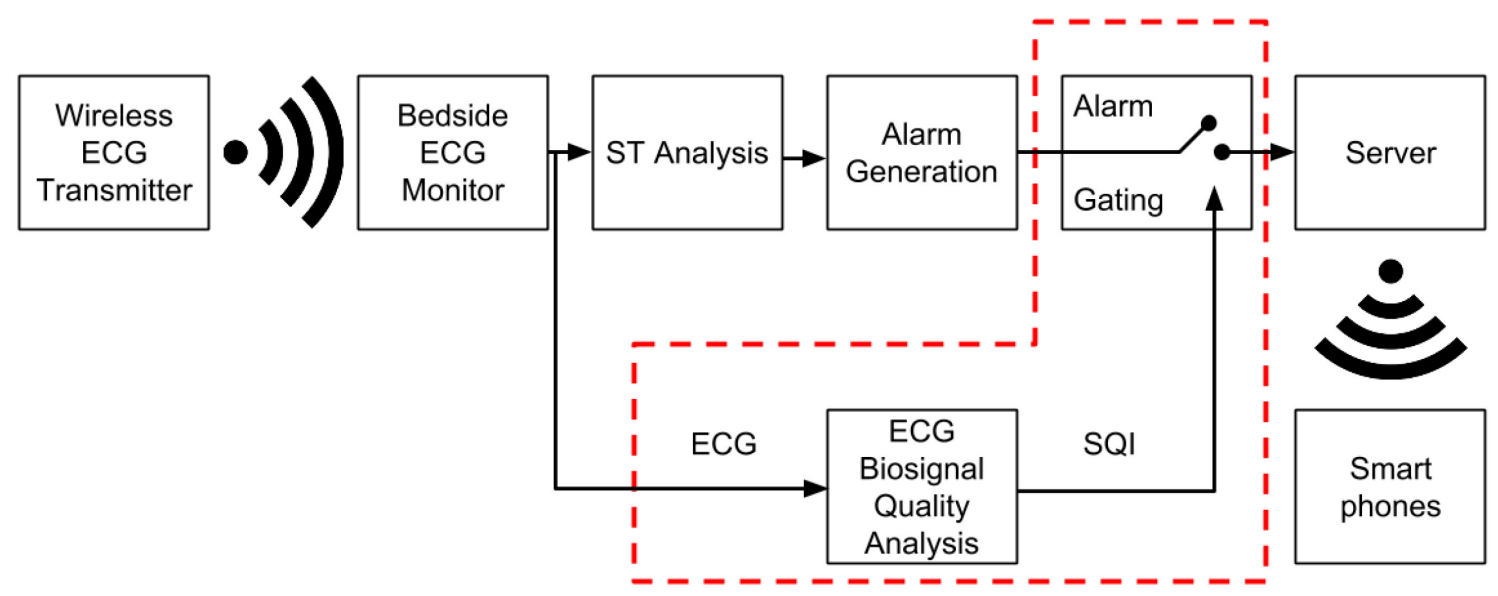

Figure 6.3 Myocardial ischemia alarm signal flow, highlighting components of $S Q V S_{A L}$ used to gate the alarm output of the alarm generating FSM

This 2 minute delay before allowing alarms through is to ensure that alarms leaving the alarm generation stage have had sufficient time to adapt to the increased signal quality. If an actual alarm occurred during a period of poor signal quality, it would continue alarming after the SQIs returned to an acceptable level, whereas, an alarm purely based on poor signal quality would stop alarming once the signal quality returned.

In this case, the SQI controlled gate has also been configured such that is does not interrupt an on-going alarm when signal quality drops. It only prevents new alarms from being passed through during periods of poor signal quality. Example operation of this configuration is shown in Figure 6.4. In this case, that FSM reached the alarming state, but the alarm was suppressed by $S Q V S_{A L}$ because of the poor signal quality. 

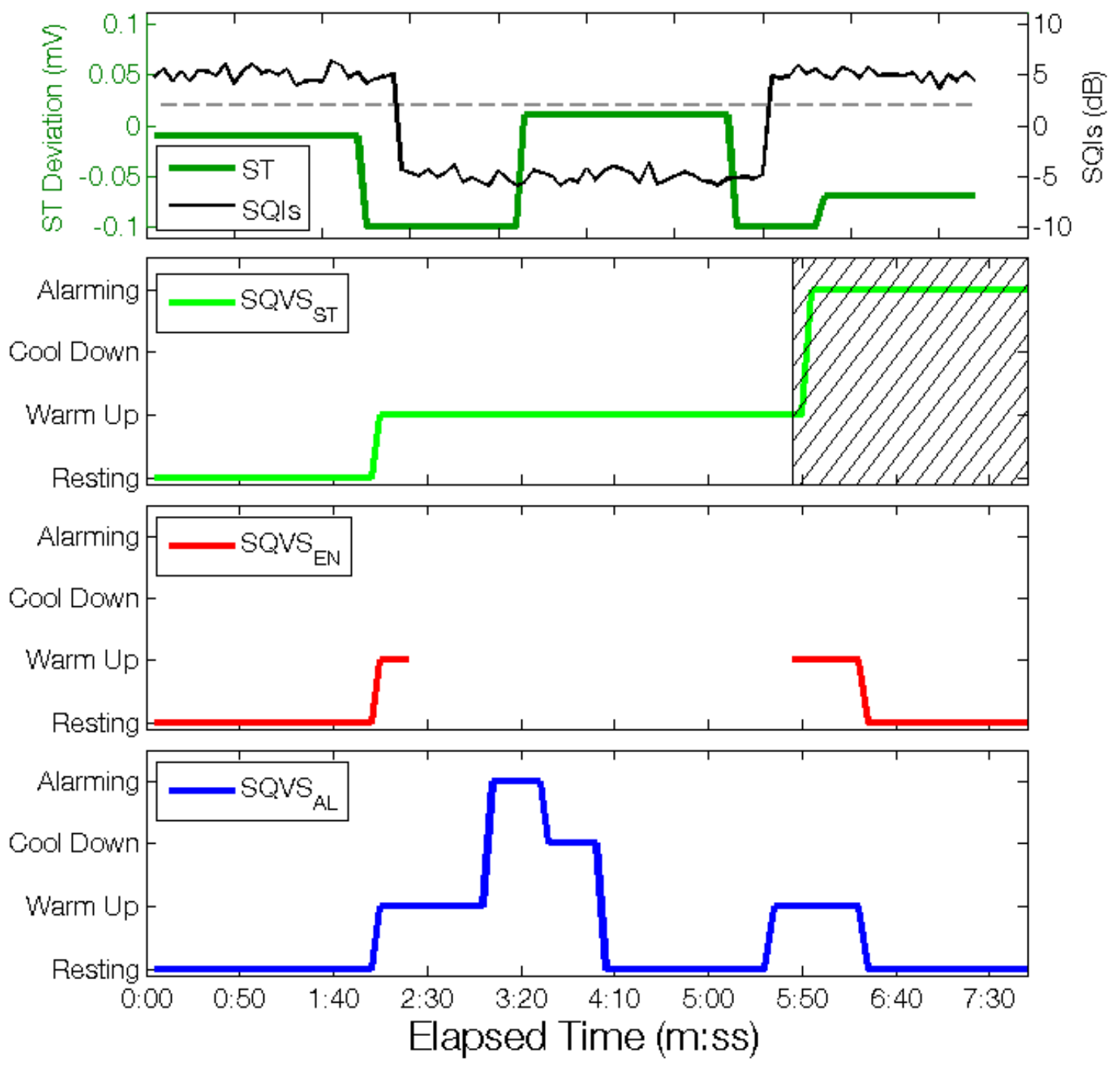

Figure 6.4 Varying responses from the three SQVS configurations to the same set of SQI and ST value inputs. Hatched sections indicate an alarm output was allowed out of the system and gray dotted line indicates SQI threshold

\subsection{Methodology}

Evaluation of the three SQVS configurations was performed using full-length ECG records from Physionet's Long-Term ST Database [39] and motion artifact from the MIT-BIH Noise Stress Test Database [74], which are both described in Section 3.3.1. Physionet's Long-Term ST Database has accompanying annotations, named '16a', 
detailing the magnitude of the ST segment deviation for every beat at various times past the $\mathrm{J}$ point (e.g., $\mathrm{J}+0 \mathrm{~ms}, \mathrm{~J}+20 \mathrm{~ms}, \mathrm{~J}+40 \mathrm{~ms}$, etc.). These ' $16 \mathrm{a}$ ' annotations were established by an international group of expert annotators from Slovenia, Italy, and the UK [39]. Using these annotations, a GS for alarms was established as described in 6.2.1. Fulllength records were then contaminated with motion artifact and played through the commercial bedside monitor to acquire ST values. These ST values were run through the three SQVS configurations at various threshold levels and the resulting alarms were compared to the GS following the statistical definitions described in Section 6.2.2. Section 6.2.3 discusses these evaluation methods in detail.

\subsubsection{Signal Selection}

Physionet's Long-Term ST Database contains 86 individual ECG records, each containing at least two leads. Of these 86 records, only those containing Lead II and V2 data were selected for further analysis. PROSE utilizes Lead II and V5 data in their analysis; however, since Lead II and V5 are not available in Physionet's Long-Term ST Database, the Lead II/V2 pair was chosen because it most closely matches the ECG projections used by PROSE. A total of 16 records match this selection criterion and are listed in Table 3.1. ST values from Physionet's '16a' annotations taken at J+60ms were analyzed with the alarm generating FSM to derive GS alarms for each of these 16 records. ST values were then acquired for each of the 16 records using the bedside monitor following the descriptions outlined in Chapter 3. These bedside monitor ST values were fed through the alarm generating FSM and the resulting alarms were compared to the GS. 
Given that Physionet's Long-Term ST Database is specifically targeted at records with varying ST deviations, some records were found to be alarming for a large proportion of the record, as illustrated in Figure 6.5, which depicts Lead II ST values taken from s20121. In this case, the entire s20121 record was found to return a steady alarm; once the alarm was initiated, ST deviation values did not return between the $-0.5 \mathrm{mV}$ and 0.5 $\mathrm{mV}$ constraints required to trigger the alarm cool down period.

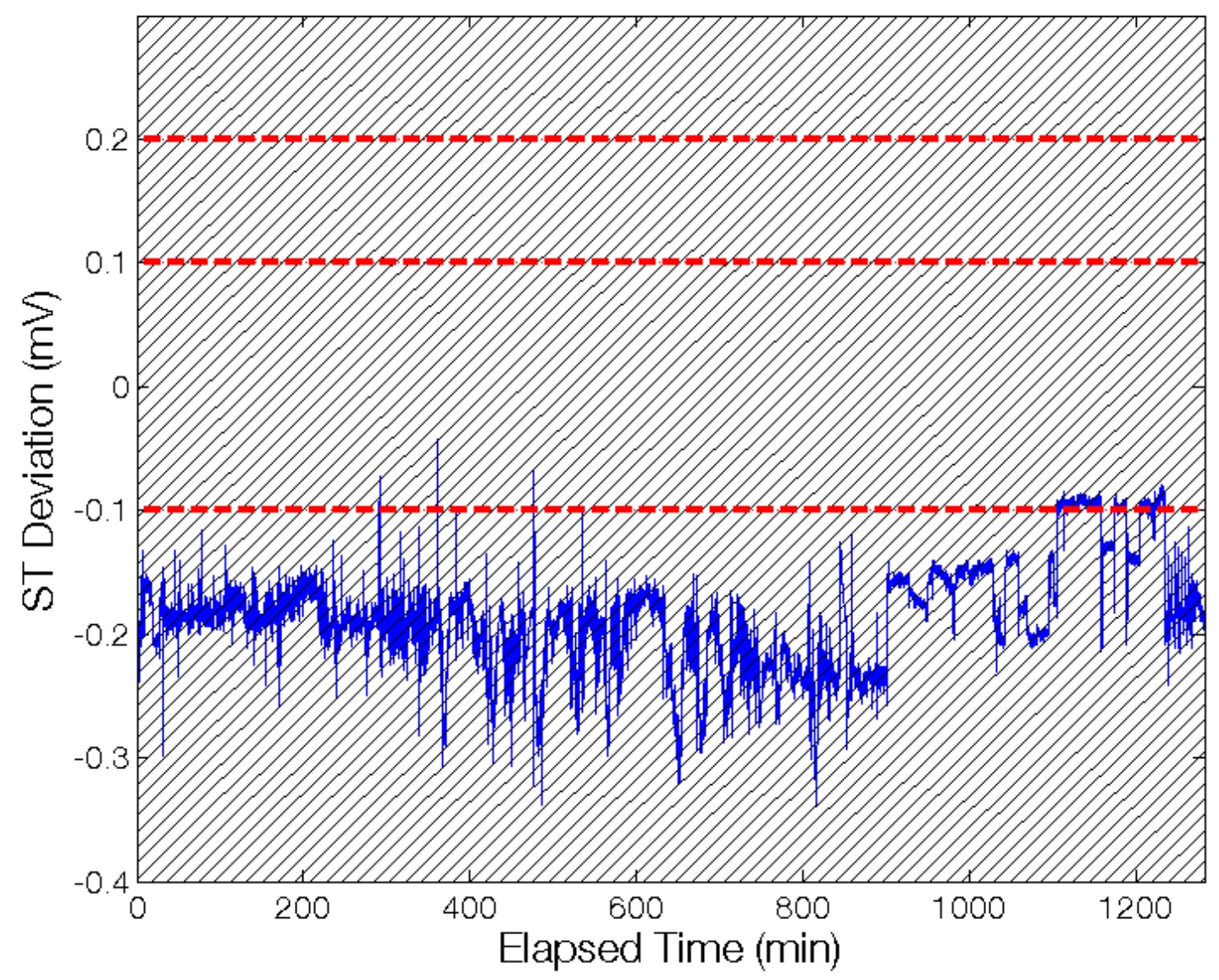

Figure 6.5 Lead II ST values from full-length 's20121' record resulting in full-length alarm. Hatched area indicates that an alarm is active.

Additionally, upon comparing the GS alarms to the bedside monitor alarms, it was found that false alarms already existed in the bedside analysis of the Physionet records prior to 
the addition of motion artifact. While some of these alarms were likely due to periods of faltering ECG signal quality within the original Physionet records, it was found that others were due to the bedside monitor miscalculating fiducial points (i.e., landmarks) in the PQRST complex. For example, the bottom of the bedside monitor screen shown in Figure 6.6 displays ST measurement sites used when it estimated and logged maximal deviations of $-2.00 \mathrm{~mm}(-0.20 \mathrm{mV})$ and $1.12 \mathrm{~mm}(0.11 \mathrm{mV})$, and from the last ischemic episode $(-1.60 \mathrm{~mm}$ or $-0.16 \mathrm{mV})$.

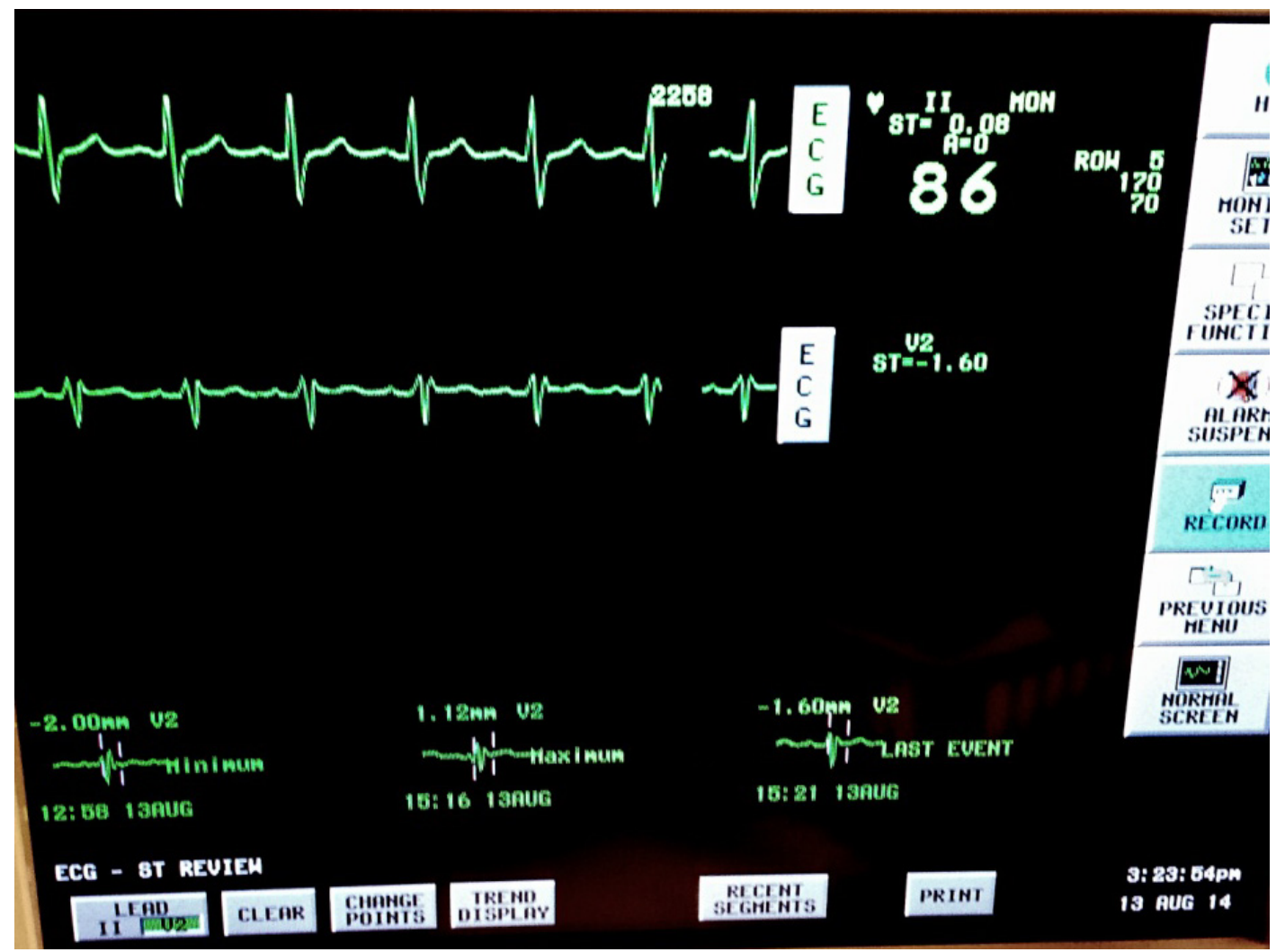

Figure 6.6 Bedside monitor showing history of recent ST values. Note how the marker indicating the ST measurement site remains steady while the marker indicating the PR segment fluctuates up and down

It can be seen that the ST segment remains relatively stationary across the example beats for all three cases, whereas the PR segment is being improperly identified and, as a result, 
is fluctuating up and down thereby artificially affecting the ST value. Consequently, the 16 records that met the Lead II/V2 selection criterion were further analyzed and only records with limited, short duration alarms and limited, if any, false positives were chosen. Figure 6.7 illustrates Lead II ST values taken from s20051 that meet these criteria. This was done to provide distinct positive alarms for a GS, to provide ample time for alarms to be generated due to additional motion artifact, and to ensure that existing false alarms due to other factors were not confounding the analysis.

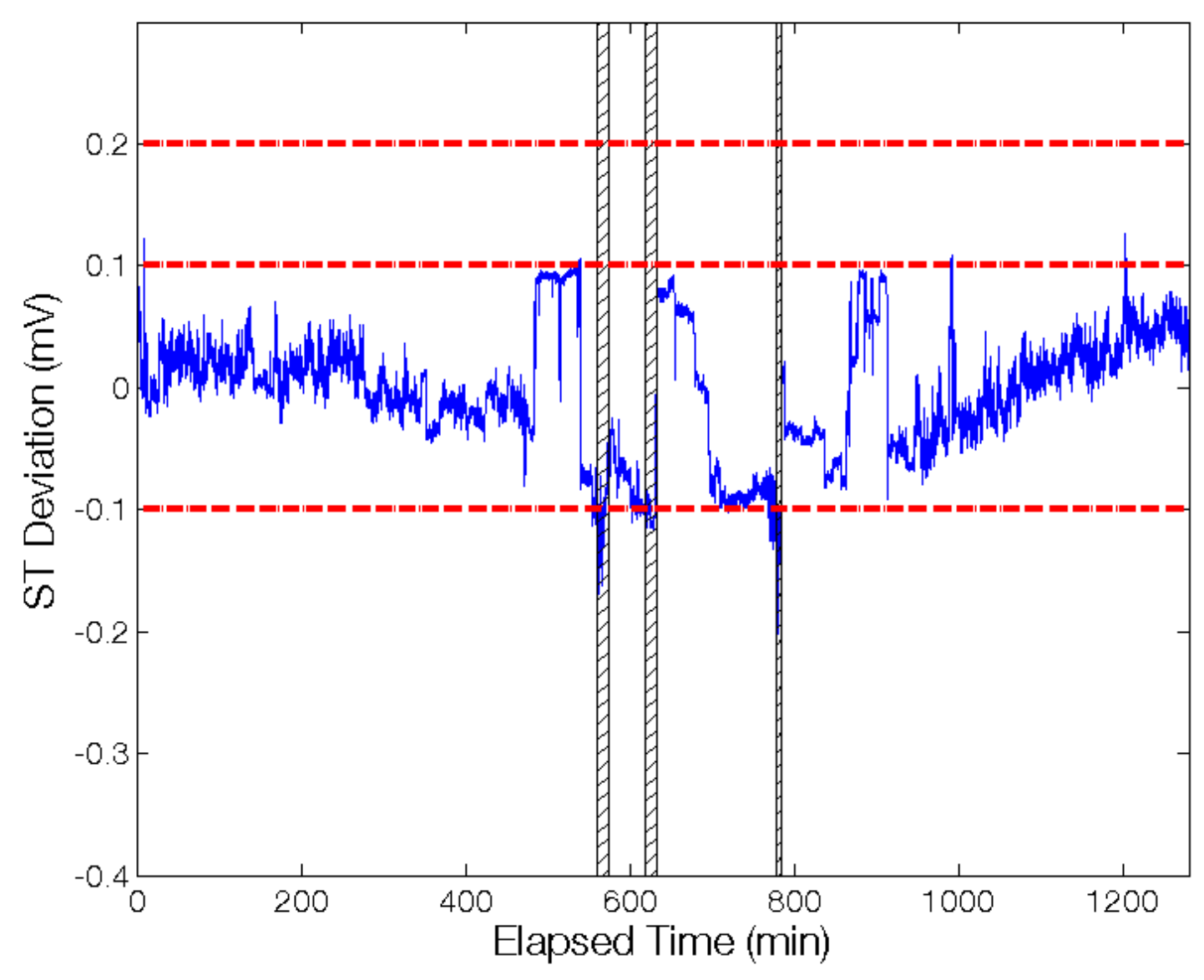

Figure 6.7 Lead II ST values from full-length s20051 record resulting in three distinct ischemia alarms. Hatched areas indicate an alarm is active 
Two records were chosen to evaluate the SQVS configurations: s20051 and s20111. Description of the alarms present on each lead and signal are included in Table 6.1.

\section{Table 6.1 Number and type of alarms occurring in records used to evaluate SQVS configurations}

\begin{tabular}{|c|c|c|c|c|c|c|}
\hline \multirow{2}{*}{ Record } & \multicolumn{6}{|c|}{ Number of alarms } \\
\cline { 2 - 7 } & \multicolumn{3}{|c|}{ Lead II } & \multicolumn{3}{c|}{ V2 } \\
\cline { 2 - 7 } & TP & FP & FN & TP & FP & FN \\
\hline \multirow{2}{*}{ s20051 } & 3 & 0 & 0 & 0 & 18 & 0 \\
\hline s20111 & 6 & 0 & 1 & 4 & 4 & 2 \\
\hline
\end{tabular}

\subsubsection{Statistical Definitions}

Before evaluating the effectiveness of these three SQVS, it was necessary to establish metrics against which to judge its performance. The ultimate goal of this research project was to increase the PPV of myocardial ischemia alarms without diminishing their sensitivity. PPV, also known as precision, is essentially the likelihood that a received alarm will be

a true alarm, and can be expressed as in (6.1).

$$
\text { Precision }=\frac{\text { Number of True Positive Alarms }}{\text { All Positive Alarms }}=\frac{T P}{T P+F P}
$$

where TP is the number of true positives (i.e., correctly identified ischemic episodes), and FP is the number of false positives (i.e., an alarm that occurred when there was no ischemic episode occurring). Alternatively, sensitivity, also known as recall, measures the ability of the generated alarms to properly identify all actual ischemic episodes within the ECG and can be written as shown in (6.2). 


$$
\text { Sensitivity }=\frac{\text { Number of True Positive Alarms }}{\text { Number of actual ischemic episodes }}=\frac{T P}{T P+F N}
$$

where $\mathrm{FN}$ is the number of false negatives (i.e., ischemic episodes that took place without generating an alarm).

These two metrics allow quantification as to the impact of the SQVS with respect to the validity of alarms that clinicians receive on their cell phones, while simultaneously ensuring the SQVS does not downgrade monitor performance. In order to make use of these metrics, TP, FP and FN must first be defined, as described below. In this research work, the SQVS alarms are being tested against a set of GS alarms. These GS alarms are derived from the Physionet Long-Term ST Database records and represent ischemic events. Records included in this GS are discussed previously, in Section 6.2.1.

This thesis work defines a TP as the detection of a single GS ischemic event by the commercial bedside monitor, operating in conjunction with the SQVS. The bedside monitor alarm may occur at any time during the GS ischemic event, so long as it occurs before the end of the ischemic event. If more than one bedside alarm is triggered by a single GS ischemic episode, only one TP is registered and subsequent alarms during the same ischemic episode are ignored; however, a single bedside alarm may detect more than one individual GS ischemic episode, resulting in multiple TP from one single bedside alarm. These three TP cases are illustrated in Figure 6.8. 


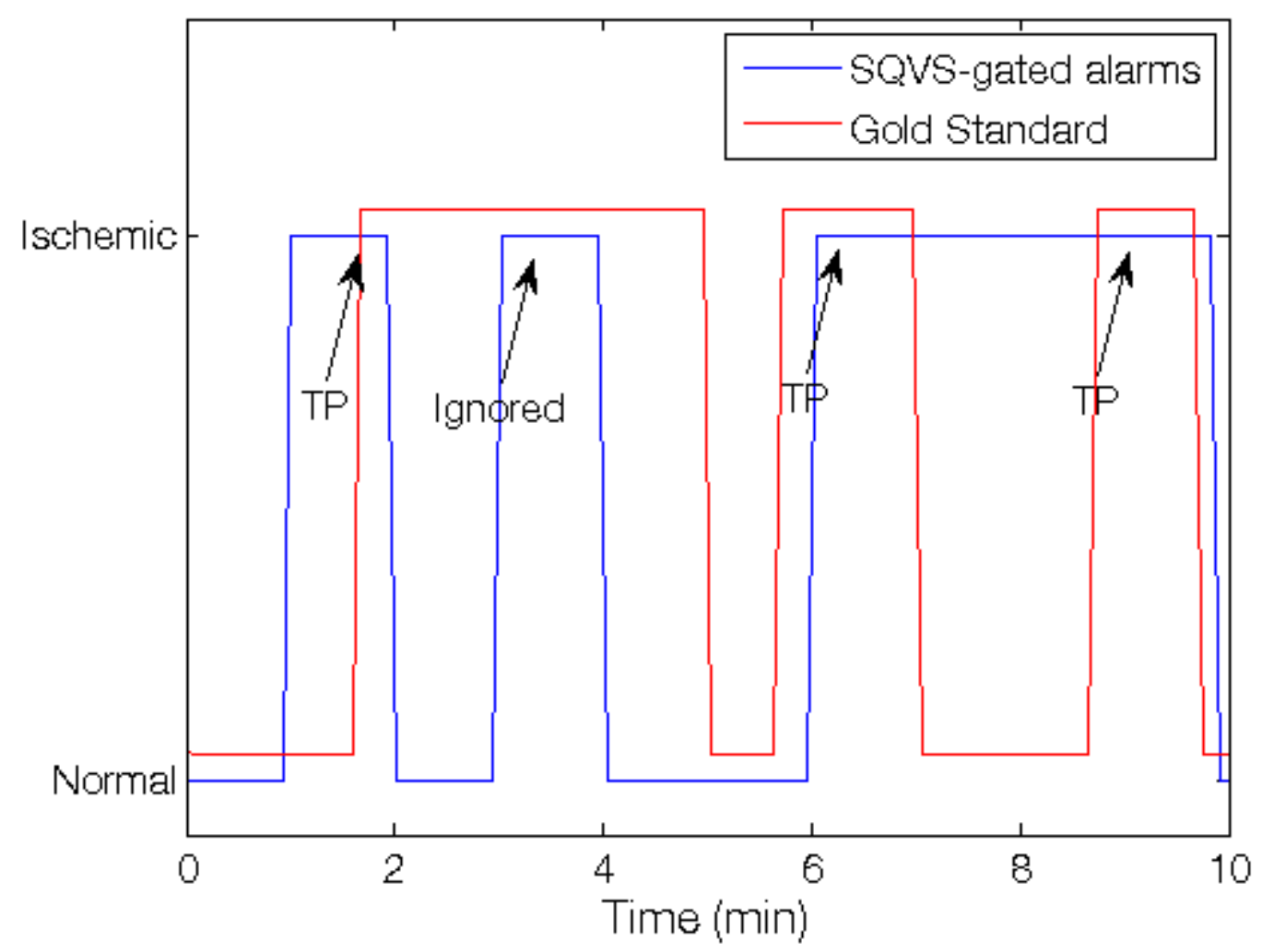

Figure 6.8 Simulated alarms and gold standard ischemic episodes to demonstrate definition of TP

This thesis work defines a FP as a bedside alarm that shares no overlap between it and a GS ischemic episode. An example FP is depicted in the center of Figure 6.9.

Lastly, this thesis work defines a FN as a GS ischemic episode that shares no overlap between it and a bedside alarm (i.e., an undetected ischemic episode). Two examples of FN are depicted on either side of Figure 6.9. 


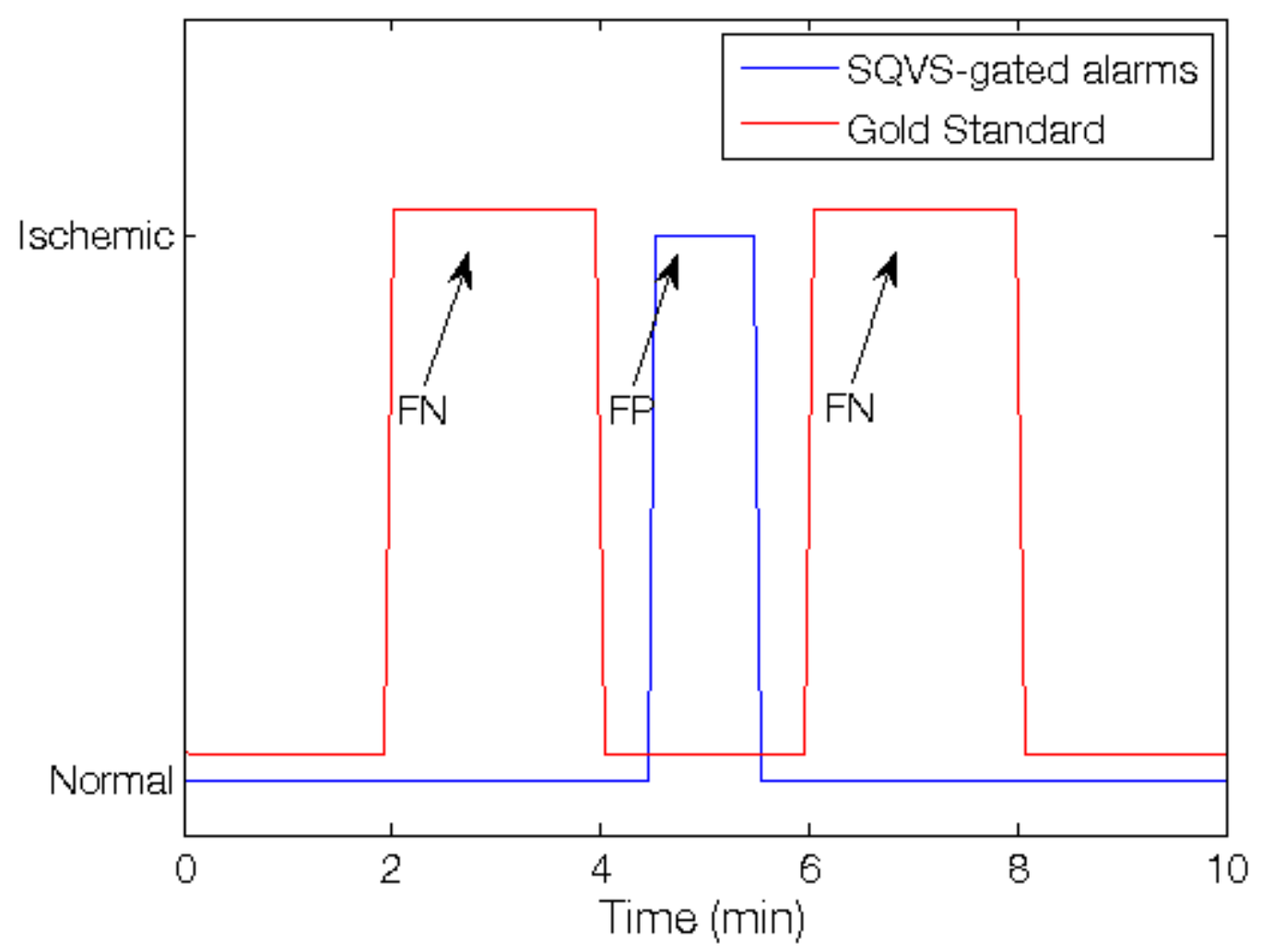

Figure 6.9 Simulated alarms and gold standard ischemic episodes to demonstrate the definition of FP and FN

Using the definitions of TP, FP and FN, it is possible to calculate the PPV and sensitivity of myocardial ischemia alarms. PPV and sensitivity provide metrics to indicate the ability of SQVS alarm gating to selectively target and reduce the occurrence of false ischemia alarms.

\subsubsection{Evaluation Methods}

\subsubsection{Generating GS Alarms}

Evaluation of these three SQVS configurations was accomplished using full-length ECG records from Physionet's Long-Term ST Database and motion artifact from the MIT-BIH Noise Stress Test Database. Physionet provides ST values (in an annotation file called 
'16a') for each beat in a given ECG record. These confirmed ST values were used to create a set of GS alarms against which to compare alarms generated from the bedside monitor.

\subsubsection{Contaminating ECG Records with Motion Artifact}

ECG data were then contaminated with motion artifact from the MIT-BIH Noise Stress Test Database to simulate patient movement while monitoring. Five minutes of motion artifact was added to the ECG record every 15 minutes. Thus, the ECG was contaminant free for 15 minutes, followed by 5 minutes of contamination. This cycle was then repeated for the duration of the ECG record. Motion artifact was not scaled before being added to the ECG. To avoid re-using the same segments of motion artifact multiple times, motion artifact was taken from the 'em' record in overlapping windows, offset from each other by 25 seconds. For example, the first segment of motion artifact added to an ECG record was taken from the 'em' record between 0 and 5 minutes, the second segment was taken from between the elapsed times of 0:25 minutes and 5:25 minutes. This allowed for 60 semi-unique segments of motion artifact to be taken from the 'em' record, which is 30 minutes in length. As mentioned previously, motion artifact in the MIT-BIH Noise Stress Test Database is sampled at $360 \mathrm{~Hz}$, while ECG records from Physionet's Long-Term ST Database are sampled at $250 \mathrm{~Hz}$. Motion artifact was consequently down-sampled to 250 $\mathrm{Hz}$ using MATLAB's resample function, which employs an anti-aliasing filter, prior to its addition to the ECG data. 


\subsubsection{Signal Routing}

Contaminated records were loaded onto a patient simulator (MS400 Multiparameter Simulator, CONTEC Medical Systems, Qinhuangdoa, Hebei Province, P. R. China), which was connected to a wireless telemetry pack (Ultraview Digital Telemetry Multiparameter Transmitter 90345, Spacelabs Medical, Issaquah, WA, USA). The patient simulator played each ECG record in real time, which was transmitted wirelessly to a commercial bedside monitor (91369 Ultraview SL Bedside Monitor, Spacelabs Medical, Issaquah, WA, USA) by the telemetry pack. ST values were recorded on a PC via COM port from the bedside monitor, which had been configured to transmit the values in 5 second intervals. Signal routing for each of these SQVS configurations is discussed in detail in Section 3.8 and diagrams of the test setups for the three configurations are shown in Figure 3.11 to Figure 3.13.

The ST values acquired from the bedside monitor were then run through an alarm generating FSM (described in Section 3.5.2), in conjunction with each SQVS configuration outlined in Section 6.1. These SQVS configurations employed SQI threshold levels between -15 and $20 \mathrm{~dB}$, in steps of $1 \mathrm{~dB}$. The SQIs used by the SQVS were generated from the contaminated ECG records using the $S Q I_{25}$ algorithm outlined in Chapter 4. Manually corrected beat annotations from Physionet ('atr') were used to identify all R peaks in the ECG during SQI generation, as explained in Section 4.2.3.1.

SQIs and the bedside generated ST values were synchronized following the synchronization procedure described in Section 3.4.4. 


\subsubsection{Generating Statistics}

Performance of each SQVS configuration was evaluated based on the PPV and sensitivity of the generated alarms. Due to the presence of FPs on the uncontaminated V2 leads (listed in Table 6.1), statistics were only generated for Lead II data (the lead actually used by PROSE). Once all alarms for a given record had been acquired, they were manually inspected for TPs, FPs and FNs. PPV and sensitivity were calculated (following descriptions in Section 6.2.2) for each SQVS configuration using all alarms from the contaminated ECG records.

\subsection{Results}

The bedside monitor's ST estimation algorithm is proprietary and appears to be dependent on when the bedside monitor is started in relation to the start of the ECG record. As such, the procedure of running contaminated ECG through the bedside monitor and generating statistics was performed twice following the steps outlined in Sections 6.2.3.3 and 6.2.3.4. Similar behaviour was observed for both of these runs. The TP, FP and FN associated with each SQVS configuration were averaged across both runs and then plotted against their corresponding SQI threshold levels in Figure 6.10. As the SQI threshold was increased, fewer and fewer FPs were obtained. These FPs plateaued using an SQI threshold around 0 to $5 \mathrm{~dB}$. This plateau indicates that there is a wide SQI margin between alarms due to poor signal quality and actual alarms. As the SQI threshold was increased further (beyond $10 \mathrm{~dB}$ ) the number of TPs declined, matched by the corresponding increase in FNs. 


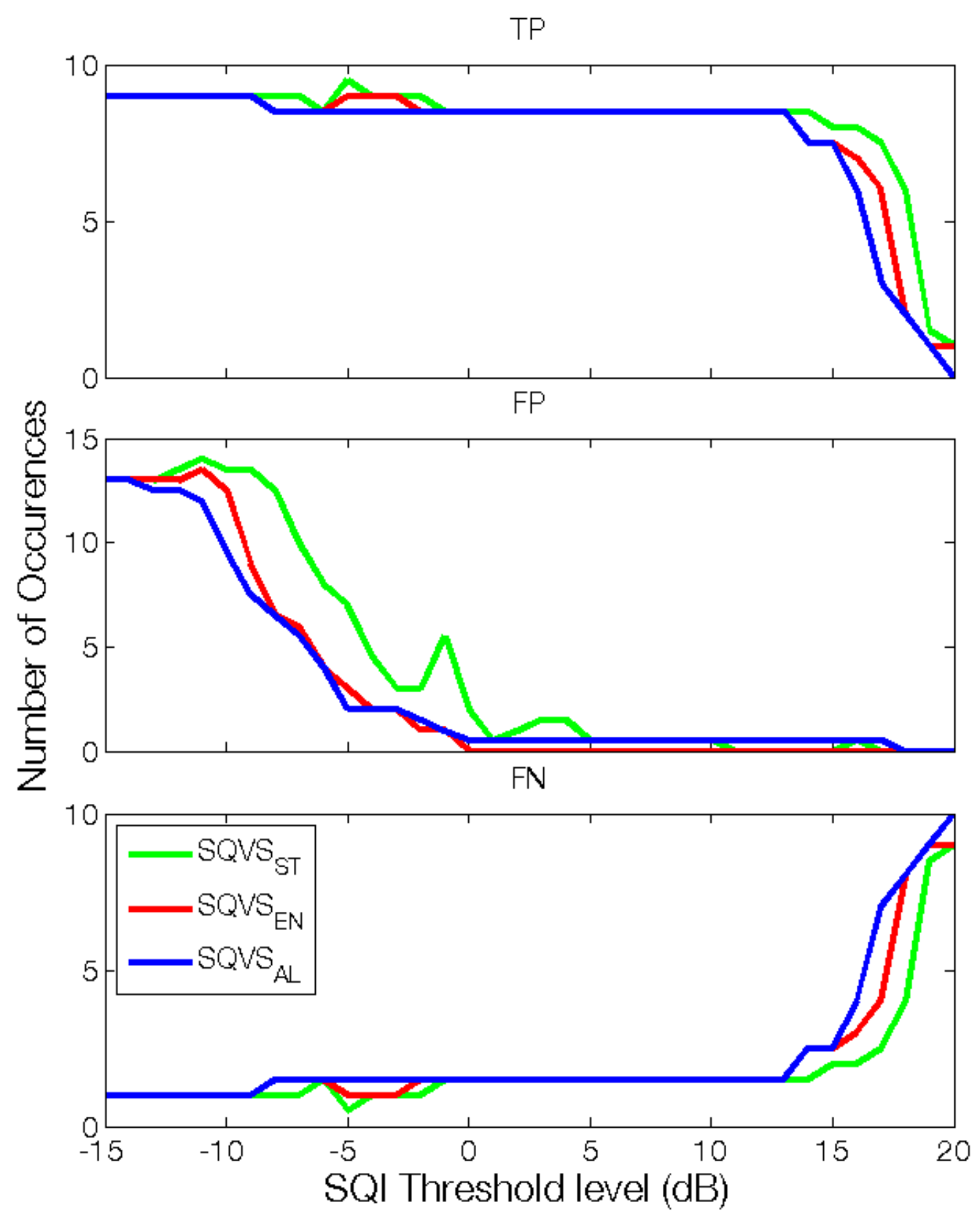

Figure 6.10 TP, FP, and FN counts for each SQVS configuration plotted against the SQI threshold at which they were attained - averaged across two runs

This decline indicates that the SQI threshold was raised too high, causing actual alarms to be ignored. TP and FP counts were relatively unresponsive for SQI thresholds below 10 dB. 
PPV and sensitivity were calculated for each SQVS configuration from these averaged alarms at each SQI threshold level and plotted in Figure 6.11 as a Precision Recall Curve (PRC). A PRC plots PPV versus sensitivity - note that PPV is synonymous with precision while sensitivity is synonymous with recall, as mentioned in Section 6.2.2.

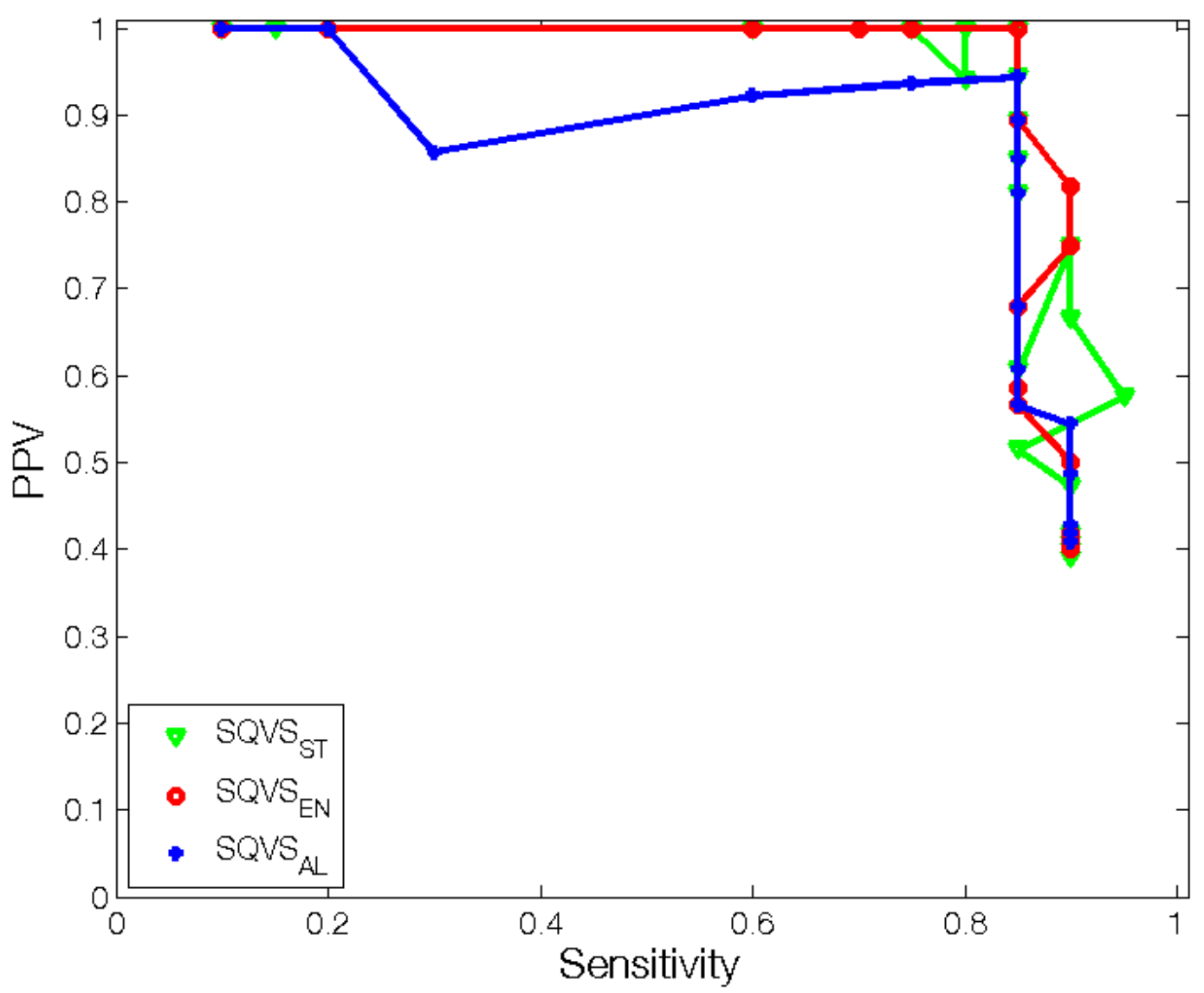

Figure 6.11 Precision Recall Curve showing PPV and sensitivity calculated from alarm counts that were averaged across two runs for three SVQS configurations using various SQI thresholds

Note that in the PRC, the bottom-rightmost point for each of the SQVS configurations represents the base case without gating from the SQVS at an SQI threshold of $-15 \mathrm{~dB}$. This base case reveals a PPV of 0.41 and sensitivity of 0.90 . The SQI threshold in these 
base cases was intentionally set lower than any SQI present in the contaminated ECG records, such that the SQVS did not perform any gating. Table 6.2 lists the highest PPV attained by each SQVS configuration without sustaining any loss to sensitivity. Alternatively, Table 6.3 lists the highest retained sensitivity for each configuration when $100 \%$ PPV was attained.

Table 6.2 Highest attained PPV without loss of sensitivity for each SQVS configuration

\begin{tabular}{|c|c|c|}
\hline Configuration & Attained PPV & SQI Threshold \\
\hline$S Q V S_{S T}$ & 0.75 & $-3 \mathrm{~dB}$ \\
\hline$S Q V S_{E N}$ & 0.82 & $-3 \mathrm{~dB}$ \\
\hline$S Q V S_{A L}$ & 0.55 & $-9 \mathrm{~dB}$ \\
\hline
\end{tabular}

Table 6.3 Highest retained sensitivity to achieve 100\% PPV for each SQVS configuration

\begin{tabular}{|c|c|c|}
\hline Configuration & Highest Sensitivity & SQI Threshold \\
\hline$S Q V S_{S T}$ & 0.85 & $11 \mathrm{~dB}$ \\
\hline$S Q V S_{E N}$ & 0.85 & $0 \mathrm{~dB}$ \\
\hline$S Q V S_{A L}$ & 0.20 & $18 \mathrm{~dB}$ \\
\hline
\end{tabular}

Statistical counts for each SQVS configuration at each SQI threshold level are included in Appendix A (first run) and Appendix B (second run). Tables A.1 and B.1 show the TP, FP, and FN counts for each SQVS configuration, while Tables A.2 and B.2 show the corresponding calculated PPV and sensitivity for the respective runs. In Appendix C, Table C.1 contains PPV and sensitivity values calculated from the averaged TP, FP, and FN alarm counts. 


\subsection{Discussion}

The end goal of the SQVS is to improve PPV while maintaining current sensitivity levels. On a PRC this objective can be visualized by trying to reach higher levels of PPV vertically, without decreasing sensitivity leftwards. All three SQVS configurations exhibited the ability to improve PPV without significant loss to the overall sensitivity. Both $S Q V S_{S T}$ and $S Q V S_{E N}$ performed comparably, while $S Q V S_{A L}$ sacrificed more sensitivity than the other two. Two ECG signals were used in this proof of concept, thus the three PRCs appear jagged because of the limited amount of data; however the overall trends are still visible. The alarm counts and resulting PPV and sensitivity values for each of the two runs through the bedside monitor returned similar results with only minor discrepancies that would not be visible in the curves shown in Figure 6.10 and Figure 6.11 .

From an implementation perspective, $S Q V S_{S T}$ and $S Q V S_{A L}$ are effectively the same system, differing only by where they were implemented in the data stream. Both are essentially just a signal gate that allows data through during periods of acceptable signal quality, where acceptability is determined by the SQI threshold. Either of these configurations can be easily implemented in conjunction with any bedside monitor, provided there is access to the raw ECG data. Implementation of $S Q V S_{E N}$ requires access to the alarm generating FSM in order to properly freeze states and timers, and is thus marginally more complicated to implement. In this case, the alarm generating FSM was implemented on the same PC logging the ST values and generating the SQIs, thus FSM access was not an issue. 
Figure 6.10 shows plateaus that span many SQI thresholds in the TP and FN plots, providing a large margin throughout which an appropriate threshold may be chosen while simultaneously safeguarding TPs and consequently, sensitivity.

These results show that using an SQVS to gate ischemia alarms on the basis of signal quality does effectively eliminate FPs resulting from motion artifact present in the ECG. Using $S Q V S_{S T}$ and $S Q V S_{E N}$, all FPs due to poor signal quality are captured before any substantial loss in TPs. One FP remains until very high SQI threshold levels when using $S Q V S_{A L}$, as can be seen in Figure 6.10 and in Appendix B. Upon investigation, this FP is generated during period a period of poor signal quality and is initially gated by $S Q V S_{A L}$; however, the FP persists beyond the two minute gating delay after signal quality is restored (described in Section 6.1.3). In this case, despite the ST values falling within acceptable boundaries $(-0.1 \mathrm{mV} \leq \mathrm{ST} \leq 0.1 \mathrm{mV})$ the alarm persisted because the $\mathrm{ST}$ values continued to remain outside the range required to trigger the end of an alarm ($0.05 \mathrm{mV} \leq \mathrm{ST} \leq 0.05 \mathrm{mV})$. For this reason, $S Q V S_{S T}$ and $S Q V S_{E N}$ perform better than $S Q V S_{A L}$. It is also worthwhile to note that $S Q V S_{E N}$ exhibits the most stable response of the three configurations, as illustrated by the smooth plateaus in Figure 6.10.

It should be noted that these results deal only with alarms generated during periods of poor signal quality; however, myocardial ischemia alarms can be subject to FPs for additional reasons, such as poor identification of the fiducial points causing artificial ST deviations (illustrated in Figure 6.6). 


\subsection{Conclusions}

SQVS-gating based on signal quality does effectively remove noise related FP alarms. $S Q V S_{S T}$ and $S Q V S_{E N}$ performed comparably and both performed better than $S Q V S_{A L}$. The $S Q V S_{E N}$ configuration exhibited the most stable response and, thus, ultimately performed the best. While these results are encouraging, more data needs to be processed to better verify functionality and performance of using the SQVS to modify ischemia alarms.

While investigating the impact of SQVS-gating, it was observed that poor signal quality is not the only cause of FP alarms. FPs were also observed at times to be directly related to misidentification of fiducial points in the ECG by the bedside monitor - a problem outside the scope of this thesis. Performing parallel ST analysis on the ECG data may help detect the occurrence of this misidentification and should be investigated. 


\section{Conclusions and Recommendations for Future Work}

\subsection{Thesis Summary}

A high false alarm rate currently renders myocardial ischemia monitoring via ambulatory ECG clinically impractical. Many of these false alarms are due to ST values derived during periods of poor ECG signal quality. This thesis contributed methods to automatically quantify ECG signal quality on a single ECG lead without the need for any supplementary data; whether from additional ECG leads or from any alternative sensors (e.g. photoplethysmography, accelerometer, arterial blood pressure). Performance of these methods was evaluated using both non-ischemic and ischemic ECG data contaminated with motion artifact. Results indicated correlation as high as $r=0.96$ between the SQI generated by these methods and known levels of noise present in the test data.

This thesis also investigated the potential of using this SQI to modify alarm output from a commercially available bedside ECG monitor. It was found that the presence of motion artifact in the ECG signal greatly increases the chance of generating a false alarm and that this motion artifact was found to be detectable by the SQI. Three SQVS configurations were investigated to modify alarm output. These configurations were evaluated by measuring the PPV and sensitivity of the modified alarms and comparing them to the unmodified, base alarms. It was found that modifying alarms on the basis of signal quality, as denoted by the SQI, effectively raises PPV without detriment to alarm sensitivity when gating alarms due to poor quality ECG. These results, while 
encouraging, should be investigated further with larger data sets to better verify functionality and performance of modifying alarms on the basis of signal quality. This is discussed further in the following section. It is also important to note that in this thesis the SQI was targeted towards reducing false myocardial ischemia alarms; however, the SQI can also find utility in other cardiac applications, such as ECG biometrics or fitness monitoring.

\subsection{Future Work}

While the proof of concept results obtained during this thesis work are encouraging, future investigation is warranted in the following areas:

\section{Investigation using larger data sets}

The methods presented in this thesis should be validated using larger data sets of ECG. Only one noise source was used in this thesis work; future work should investigate performance of these methods using supplementary motion artifact records. Performance of these methods should also be investigated in the presence of additional types of ECG contaminants, such as more severe baseline wander, power line interference and muscle artifacts. Additionally, different ECG leads should be investigated to establish the robustness of these methods across varying ECG projections. Ideally this investigation should include data that is more representative of PROSE monitoring conditions. This investigation could potentially be performed using previously collected PROSE ECG data. 


\section{Alternative SQI metrics}

Methods presented in this thesis to assess and quantify signal quality focused on SNR where signal power is defined by RMS values. Additional methods of deriving signal quality (e.g. autocorrelation) should be investigated and compared to the methods presented in this thesis.

\section{Detection of false alarms due to causes other than poor signal quality}

These methods focus on increasing PPV of ischemia alarms by eliminating false alarms generated during periods of poor signal quality. It was discovered that poor signal quality is not the sole source of false alarms. Misidentification of ECG fiducial points (such as the PR segment) by the bedside monitor also has the potential to trigger false alarms. Future work should investigate the ability to discern misidentification of these fiducial points.

\section{Real-time implementation}

Future work should ultimately investigate real-time implementation of these methods for patient monitoring. Increased PPV of myocardial ischemia detection may pave the way for potential home monitoring of postoperative patients. When coupled with today's laparoscopic surgery techniques, this could allow for early discharge following risk stratification.

\section{Ischemia detection via machine learning}

Currently, clinical ischemia detection via ECG follows a somewhat heuristic approach. In automated systems, it has been more or less simplified to a set of rules used to interpret 
what culminates in an ST value; however there are many nuances to the ST segment that guide actual clinical interpretation. Therefore, future work should also consider employing machine learning algorithms for ST analysis for the detection of ischemia and, thus, focus on the extraction of various features from the ST segment (e.g., various ST morphologies such as ST slope, ST length, etc.). 


\section{References}

[1] P. J. Devereaux et al., "Effects of extended-release metoprolol succinate in patients undergoing non-cardiac surgery (POISE trial): a randomised controlled trial," The Lancet, vol. 371, no. 9627, pp. 1839-1847, May 2008.

[2] P. J. Devereaux, "Perioperative cardiac events in patients undergoing noncardiac surgery: a review of the magnitude of the problem, the pathophysiology of the events and methods to estimate and communicate risk," Can. Med. Assoc. J., vol. 173, no. 6, pp. 627-634, Sep. 2005.

[3] F. Badilini et al., "Beat-to-beat quantification and analysis of ST displacement from Holter ECGs: a new approach to ischemia detection," in Proceedings of Computers in Cardiology, 1992, pp. 179-182.

[4] P. T. O'Gara et al., "2013 ACCF/AHA guideline for the management of STelevation myocardial infarction: executive summary a report of the American college of cardiology foundation/American Heart Association task force on practice guidelines," Circulation, vol. 127, no. 4, pp. 529-555, Jan. 2013.

[5] B. Haider and H. Yang, "PROSE 3: Early ischemia detection using digitized ST values," in Rehabilitation Engineering and Assistive Technology Society of North America (RESNA) Conference, Toronto, ON, 2011.

[6] M. Cvach, "Monitor alarm fatigue: an integrative review," Biomed. Instrum. Technol., vol. 46, no. 4, pp. 268-277, Jul. 2012.

[7] ECRI Institute, "Top 10 health technology hazards for 2014," Health Devices, vol. 42, no. 11, Nov. 2013.

[8] Raby KE et al., "Detection and significance of intraoperative and postoperative myocardial ischemic in peripheral vascular surgery," JAMA, vol. 268, no. 2, pp. 222-227, Jul. 1992.

[9] G. Landesberg et al., "Myocardial infarction after vascular surgery: the role of prolonged, stress-induced, ST depression-type ischemia," J. Am. Coll. Cardiol., vol. 37, no. 7, pp. 1839-1845, Jun. 2001.

[10] D. Poldermans et al., "Guidelines for pre-operative cardiac risk assessment and perioperative cardiac management in non-cardiac surgery," Eur. Heart J., vol. 30, no. 22, pp. 2769-2812, Nov. 2009.

[11] K. E. Fleischmann et al., "Association between cardiac and noncardiac complications in patients undergoing noncardiac surgery: outcomes and effects on length of stay," Am. J. Med., vol. 115, no. 7, pp. 515-520, Nov. 2003. 
[12] J. B. Dimick et al., "Hospital costs associated with surgical complications: A report from the private-sector National Surgical Quality Improvement Program," J. Am. Coll. Surg., vol. 199, no. 4, pp. 531-537, Oct. 2004.

[13] J. Starr, "Hospital acquired infection," BMJ, vol. 334, no. 7596, pp. 708-708, Apr. 2007.

[14] E. J. Topol et al., "A randomized controlled trial of hospital discharge three days after myocardial infarction in the era of reperfusion," N. Engl. J. Med., vol. 318, no. 17, pp. 1083-1088, Apr. 1988.

[15] T. H. Lee et al., "Derivation and prospective validation of a simple index for prediction of cardiac risk of major noncardiac surgery," Circulation, vol. 100, no. 10, pp. 1043-1049, Sep. 1999.

[16] E. A. Ashley and J. Niebauer, "Conquering the ECG," 2004. [Online]. Available: http://www.ncbi.nlm.nih.gov/books/NBK2214/. [Accessed: 13-Nov-2013].

[17] B. Blaus, "Blausen 0462 HeartAnatomy" - Own work. Licensed under Creative Commons Attribution 3.0 via Wikimedia Commons - Available from: http://commons.wikimedia.org/wiki/File:Blausen_0462_HeartAnatomy.png\#mediav iewer/File:Blausen_0462_HeartAnatomy.png. .

[18] J. G. Webster, Medical Instrumentation Application and Design, 4th ed. Wiley, 2011.

[19] J. Malmivuo, Bioelectromagnetism: Principles and Applications of Bioelectric and Biomagnetic Fields, 1 edition. New York: Oxford University Press, 1995.

[20] P. Kligfield et al., "Recommendations for the standardization and interpretation of the electrocardiogram part I: the electrocardiogram and its technology," Circulation, vol. 115, no. 10, pp. 1306-1324, Mar. 2007.

[21] J. F. Thayer et al., "A meta-analysis of heart rate variability and neuroimaging studies: implications for heart rate variability as a marker of stress and health," Neurosci. Biobehav. Rev., vol. 36, no. 2, pp. 747-756, Feb. 2012.

[22] J. Pan and W. J. Tompkins, "A real-time QRS detection algorithm," IEEE Trans. Biomed. Eng., vol. BME-32, no. 3, pp. 230-236, Mar. 1985.

[23] Z.-E. Hadj Slimane and A. Naït-Ali, "QRS complex detection using empirical mode decomposition,” Digit. Signal Process., vol. 20, no. 4, pp. 1221-1228, Jul. 2010.

[24] Z. Zidelmal et al., "QRS detection based on wavelet coefficients," Comput. Methods Programs Biomed., vol. 107, no. 3, pp. 490-496, Sep. 2012. 
[25] M. H. Crawford et al., "ACC/AHA guidelines for ambulatory electrocardiography: executive summary and recommendations," Circulation, vol. 100, no. 8, pp. 886893, Aug. 1999.

[26] J. C. Huhta and J. G. Webster, "60-Hz interference in electrocardiography," IEEE Trans. Biomed. Eng., vol. BME-20, no. 2, pp. 91-101, Mar. 1973.

[27] J. G. Webster, "Interference and motion artifact in biopotentials," in Region Six Conference Record, 1977. IEEE 1977, 1977, pp. 53-64.

[28] L. T. Sheffield et al., "AHA special report. Recommendations for standards of instrumentation and practice in the use of ambulatory electrocardiography.," Circulation, vol. 71, no. 3, p. 626A-636A, Mar. 1985.

[29] G. D. Clifford et al., Advanced Methods And Tools for ECG Data Analysis. Norwood, MA, USA: Artech House, Inc., 2006.

[30] N. V. Thakor et al., "Estimation of QRS complex power spectra for design of a QRS filter," IEEE Trans. Biomed. Eng., vol. BME-31, no. 11, pp. 702-706, Nov. 1984.

[31] A. Ruha et al., "A real-time microprocessor QRS detector system with a 1-ms timing accuracy for the measurement of ambulatory HRV," IEEE Trans. Biomed. Eng., vol. 44, no. 3, pp. 159-167, Mar. 1997.

[32] I. I. Christov and I. K. Daskalov, "Filtering of electromyogram artifacts from the electrocardiogram," Med. Eng. Phys., vol. 21, no. 10, pp. 731-736, Dec. 1999.

[33] Iskandrian AS et al., "Asymptomatic myocardial ischemia," Arch. Intern. Med., vol. 141, no. 1, pp. 95-97, Jan. 1981.

[34] W. E. Samson and A. M. Scher, "Mechanism of ST-segment alteration during acute myocardial injury," Circ. Res., vol. 8, no. 4, pp. 780-787, Jul. 1960.

[35] A. G. Kléber, "ST-segment elevation in the electrocardiogram: a sign of myocardial ischemia," Cardiovasc. Res., vol. 45, no. 1, pp. 111-118, Jan. 2000.

[36] P. M. Rautaharju et al., "AHA/ACCF/HRS Recommendations for the standardization and interpretation of the electrocardiogram part IV: the ST segment, $\mathrm{T}$ and U waves, and the QT interval," Circulation, vol. 119, no. 10, pp. e241-e250, Mar. 2009.

[37] J. Adams et al., "Earliest electrocardiographic evidence of myocardial infarction: implications for thrombolytic treatment. The GREAT Group.," BMJ, vol. 307, no. 6901, p. 409, Aug. 1993. 
[38] A. L. Goldberger et al., "PhysioBank, PhysioToolkit, and PhysioNet components of a new research resource for complex physiologic signals," Circulation, vol. 101, no. 23, pp. e215-e220, Jun. 2000.

[39] F. Jager et al., "Long-term ST database: a reference for the development and evaluation of automated ischaemia detectors and for the study of the dynamics of myocardial ischaemia," Med. Biol. Eng. Comput., vol. 41, no. 2, pp. 172-182, 2003.

[40] P. M. Okin et al., "Effect of ST segment measurement point on performance of standard and heart rate-adjusted ST segment criteria for the identification of coronary artery disease," Circulation, vol. 84, no. 1, pp. 57-66, Jul. 1991.

[41] R. D. Dony, "Karhunen-Loève Transform," in The Transform and Data Compression Handbook, 1st ed., K. R. Rao and P. C. Yip, Eds. CRC Press, 2000.

[42] P. Laguna et al., "Karhunen-Loève transform as a tool to analyze the ST-segment. Comparison with QT interval," J. Electrocardiol., vol. 28 Suppl, pp. 41-49, 1995.

[43] P. Laguna et al., "Analysis of the ST-T complex of the electrocardiogram using the Karhunen-Loeve transform: adaptive monitoring and alternans detection," Med. Biol. Eng. Comput., vol. 37, no. 2, pp. 175-189, Mar. 1999.

[44] F. Jager et al., "Analysis of transient ST segment changes during ambulatory monitoring using the Karhunen-Loeave transform," in Proceedings of Computers in Cardiology 1992, 1992, pp. 691-694.

[45] G. Landesberg et al., "Perioperative myocardial ischemia and infarction: identification by continuous 12-lead electrocardiogram with online ST-segment monitoring," Anesthesiology, vol. 96, no. 2, pp. 264-270, Feb. 2002.

[46] S. D. Kristensen et al., "2014 ESC/ESA guidelines on non-cardiac surgery: cardiovascular assessment and management” Eur. Heart J., p. ehu282, Aug. 2014.

[47] B. J. Drew and M. G. Adams, "Clinical consequences of ST-segment changes caused by body position mimicking transient myocardial ischemia: hazards of STsegment monitoring?," J. Electrocardiol., vol. 34, no. 3, pp. 261-264, Jul. 2001.

[48] H. Yang, "Personal Communication," 15-Jul-2014.

[49] H. Yang et al., "PROSE 3 (Perioperative Ischemia Reduction): Customized Alarm from Logged ST Values," presented at the Ottawa Hospital Research Institute, Ottawa, ON.

[50] W. Einthoven, "The telecardiogram," Am. Heart J., vol. 53, no. 4, pp. 602-615, Apr. 1957. 
[51] N. H. Lovell et al., "Biosignal quality detection: an essential feature for unsupervised telehealth applications," in 2010 12th IEEE International Conference on e-Health Networking Applications and Services (Healthcom), 2010, pp. 81-85.

[52] N. H. Lovell and S. J. Redmond, "Biosignal Processing to Meet the Emerging Needs of Telehealth Monitoring Environments," in Advances in Biomedical Sensing, Measurements, Instrumentation and Systems, S. C. Mukhopadhyay and A. Lay-Ekuakille, Eds. Springer Berlin Heidelberg, 2010, pp. 263-280.

[53] Y. Xie et al., "Effect of ECG quality measures on piecewise-linear trend detection for telehealth decision support systems," in 2010 Annual International Conference of the IEEE Engineering in Medicine and Biology Society (EMBC), 2010, pp. $2877-$ 2880.

[54] G. Paré et al., "Systematic review of home telemonitoring for chronic diseases: the evidence base," J. Am. Med. Inform. Assoc., vol. 14, no. 3, pp. 269-277, May 2007.

[55] S. J. Redmond et al., "ECG recording and rhythm analysis for distributed health care environments," in Distributed Diagnosis and Home Healthcare, R. U. Acharya and T. Tamura, Eds. New York, NY, USA: American Scientific, 2009.

[56] G. D. Fraser et al., "Automated biosignal quality analysis for electromyography using a one-class support vector machine," IEEE Trans. Instrum. Meas., vol. Early Access Online, 2014.

[57] A. D. C. Chan et al., "Wavelet distance measure for person identification using electrocardiograms," IEEE Trans. Instrum. Meas., vol. 57, no. 2, pp. 248-253, Feb. 2008 .

[58] A. Fasano et al., "Fast ECG baseline wander removal preserving the ST segment," in Proceedings of the 4th International Symposium on Applied Sciences in Biomedical and Communication Technologies, New York, NY, USA, 2011, pp. 56:1-56:5.

[59] B. Ko et al., "Motion artifact reduction in electrocardiogram using adaptive filtering based on half cell potential monitoring," in 2012 Annual International Conference of the IEEE Engineering in Medicine and Biology Society (EMBC), 2012, pp. 15901593.

[60] J. Behar et al., "ECG signal quality during arrhythmia and its application to false alarm reduction," IEEE Trans. Biomed. Eng., vol. 60, no. 6, pp. 1660-1666, 2013.

[61] C. Yu et al., "A method for automatic identification of reliable heart rates calculated from ECG and PPG waveforms," J. Am. Med. Inform. Assoc. JAMIA, vol. 13, no. 3, pp. 309-320, Feb. 2006. 
[62] J. Y. Wang, "A new method for evaluating ECG signal quality for multi-lead arrhythmia analysis," in Computers in Cardiology, 2002, 2002, pp. 85-88.

[63] J. Lee et al., "Automatic motion and noise artifact detection in Holter ECG data using empirical mode decomposition and statistical approaches," IEEE Trans. Biomed. Eng., vol. 59, no. 6, pp. 1499-1506, Jun. 2012.

[64] I. Jekova et al., "Threshold-based system for noise detection in multilead ECG recordings," Physiol. Meas., vol. 33, no. 9, p. 1463, Sep. 2012.

[65] G. D. Clifford et al., "Signal quality indices and data fusion for determining acceptability of electrocardiograms collected in noisy ambulatory environments," in Computing in Cardiology, 2011, pp. 285-288.

[66] Q. Li et al., "Artificial arterial blood pressure artifact models and an evaluation of a robust blood pressure and heart rate estimator," Biomed. Eng. OnLine, vol. 8, no. 1, pp. 1-15, Dec. 2009.

[67] Q. Li and G. D. Clifford, "Suppress false arrhythmia alarms of ICU monitors using heart rate estimation based on combined arterial blood pressure and ECG analysis," in The 2nd International Conference on Bioinformatics and Biomedical Engineering, 2008. ICBBE 2008, 2008, pp. 2185-2187.

[68] I. Silva et al., "Improving the quality of ECGs collected using mobile phones: the PhysioNet/Computing in Cardiology Challenge 2011," in Computing in Cardiology, 2011, 2011, pp. 273-276.

[69] C. Liu et al., "Real-time signal quality assessment for ECGs collected using mobile phones," in Computing in Cardiology, 2011, 2011, pp. 357-360.

[70] H. Naseri and M. R. Homaeinezhad, "Electrocardiogram signal quality assessment using an artificially reconstructed target lead," Comput. Methods Biomech. Biomed. Engin., vol. 0, no. 0, pp. 1-16, 0.

[71] F. J. Martinez-Tabares et al., "Improvement of ECG signal quality measurement using correlation and diversity-based approaches," in 2012 Annual International Conference of the IEEE Engineering in Medicine and Biology Society (EMBC), 2012, pp. 4295-4298.

[72] S. J. Redmond et al., "Electrocardiogram signal quality measures for unsupervised telehealth environments," Physiol. Meas., vol. 33, no. 9, p. 1517, Sep. 2012.

[73] S. J. Redmond et al., "ECG quality measures in telecare monitoring," in 30th Annual International Conference of the IEEE Engineering in Medicine and Biology Society, 2008, pp. 2869-2872. 
[74] G. B. Moody et al., "A noise stress test for arrhythmia detectors," presented at the Computers in Cardiology, 1984, vol. 11, pp. 381-384.

[75] G. Wehr et al., "A vector-based, 5-electrode, 12-lead monitoring ECG (EASI) is equivalent to conventional 12-lead ECG for diagnosis of acute coronary syndromes," J. Electrocardiol., vol. 39, no. 1, pp. 22-28, Jan. 2006.

[76] P. E. McSharry et al., "A dynamical model for generating synthetic electrocardiogram signals," IEEE Trans. Biomed. Eng., vol. 50, no. 3, pp. 289-294, Mar. 2003.

[77] Spacelabs Healthcare, "ST Analysis," in Ultraview SL Operations Manual, Rev. AB, Issaquah, WA, 2011.

[78] S. Suppappola and Y. Sun, "Nonlinear transforms of ECG signals for digital QRS detection: a quantitative analysis," IEEE Trans. Biomed. Eng., vol. 41, no. 4, pp. 397-400, Apr. 1994.

[79] M. L. Simoons and P. G. Hugenholtz, "Gradual changes of ECG waveform during and after exercise in normal subjects.," Circulation, vol. 52, no. 4, pp. 570-577, Oct. 1975.

[80] V. U. Medina, R. Gonzalez-Camarena, and J. C. Echeverria, "Effect of noise sources on the averaged PQRST morphology," in Computers in Cardiology, 2005, 2005, pp. 743-746.

[81] J. L. Semmlow, Biosignal and Medical Image Processing. CRC Press, 2004. 


\section{Appendix A: Statistical Counts - First Run}

\section{A.1 TP, FP, and FN counts for alarms modified at varying SQI threshold levels by each SQVS configuration}

\begin{tabular}{|c|c|c|c|c|c|c|c|c|c|}
\hline \multirow{2}{*}{$\begin{array}{c}\text { SQI } \\
\text { Threshold }\end{array}$} & \multicolumn{3}{|c|}{$S Q V S_{S T}$} & \multicolumn{3}{|c|}{$S Q V S_{E N}$} & \multicolumn{3}{|c|}{$S Q V S_{A L}$} \\
\hline & TP & FP & FN & TP & FP & FN & TP & FP & FN \\
\hline$-15 \mathrm{~dB}$ & 9 & 14 & 1 & 9 & 14 & 1 & 9 & 14 & 1 \\
\hline$-14 \mathrm{~dB}$ & 9 & 14 & 1 & 9 & 14 & 1 & 9 & 14 & 1 \\
\hline$-13 \mathrm{~dB}$ & 9 & 14 & 1 & 9 & 14 & 1 & 9 & 13 & 1 \\
\hline$-12 \mathrm{~dB}$ & 9 & 14 & 1 & 9 & 14 & 1 & 9 & 13 & 1 \\
\hline$-11 \mathrm{~dB}$ & 9 & 14 & 1 & 9 & 14 & 1 & 9 & 13 & 1 \\
\hline$-10 \mathrm{~dB}$ & 9 & 15 & 1 & 9 & 14 & 1 & 9 & 12 & 1 \\
\hline$-9 \mathrm{~dB}$ & 9 & 14 & 1 & 9 & 11 & 1 & 9 & 8 & 1 \\
\hline$-8 \mathrm{~dB}$ & 9 & 14 & 1 & 8 & 7 & 2 & 8 & 7 & 2 \\
\hline$-7 \mathrm{~dB}$ & 9 & 9 & 1 & 8 & 6 & 2 & 8 & 6 & 2 \\
\hline$-6 \mathrm{~dB}$ & 8 & 9 & 2 & 8 & 5 & 2 & 8 & 5 & 2 \\
\hline$-5 \mathrm{~dB}$ & 10 & 8 & 0 & 9 & 4 & 1 & 8 & 2 & 2 \\
\hline$-4 \mathrm{~dB}$ & 9 & 4 & 1 & 9 & 2 & 1 & 8 & 2 & 2 \\
\hline$-3 \mathrm{~dB}$ & 9 & 2 & 1 & 9 & 2 & 1 & 8 & 2 & 2 \\
\hline$-2 \mathrm{~dB}$ & 9 & 3 & 1 & 8 & 1 & 2 & 8 & 1 & 2 \\
\hline$-1 \mathrm{~dB}$ & 8 & 5 & 2 & 8 & 1 & 2 & 8 & 0 & 2 \\
\hline $0 \mathrm{~dB}$ & 8 & 2 & 2 & 8 & 0 & 2 & 8 & 0 & 2 \\
\hline $1 \mathrm{~dB}$ & 8 & 1 & 2 & 8 & 0 & 2 & 8 & 0 & 2 \\
\hline $2 \mathrm{~dB}$ & 8 & 2 & 2 & 8 & 0 & 2 & 8 & 0 & 2 \\
\hline $3 \mathrm{~dB}$ & 8 & 2 & 2 & 8 & 0 & 2 & 8 & 0 & 2 \\
\hline $4 \mathrm{~dB}$ & 8 & 2 & 2 & 8 & 0 & 2 & 8 & 0 & 2 \\
\hline $5 \mathrm{~dB}$ & 8 & 1 & 2 & 8 & 0 & 2 & 8 & 0 & 2 \\
\hline $6 \mathrm{~dB}$ & 8 & 1 & 2 & 8 & 0 & 2 & 8 & 0 & 2 \\
\hline $7 \mathrm{~dB}$ & 8 & 1 & 2 & 8 & 0 & 2 & 8 & 0 & 2 \\
\hline $8 \mathrm{~dB}$ & 8 & 1 & 2 & 8 & 0 & 2 & 8 & 0 & 2 \\
\hline $9 \mathrm{~dB}$ & 8 & 1 & 2 & 8 & 0 & 2 & 8 & 0 & 2 \\
\hline $10 \mathrm{~dB}$ & 8 & 1 & 2 & 8 & 0 & 2 & 8 & 0 & 2 \\
\hline $11 \mathrm{~dB}$ & 8 & 0 & 2 & 8 & 0 & 2 & 8 & 0 & 2 \\
\hline $12 \mathrm{~dB}$ & 8 & 0 & 2 & 8 & 0 & 2 & 8 & 0 & 2 \\
\hline $13 \mathrm{~dB}$ & 8 & 0 & 2 & 8 & 0 & 2 & 8 & 0 & 2 \\
\hline $14 \mathrm{~dB}$ & 8 & 0 & 2 & 7 & 0 & 3 & 7 & 0 & 3 \\
\hline $15 \mathrm{~dB}$ & 8 & 0 & 2 & 7 & 0 & 3 & 7 & 0 & 3 \\
\hline $16 \mathrm{~dB}$ & 8 & 1 & 2 & 7 & 0 & 3 & 6 & 0 & 4 \\
\hline $17 \mathrm{~dB}$ & 7 & 0 & 3 & 6 & 0 & 4 & 3 & 0 & 7 \\
\hline $18 \mathrm{~dB}$ & 6 & 0 & 4 & 2 & 0 & 8 & 2 & 0 & 8 \\
\hline $19 \mathrm{~dB}$ & 1 & 0 & 9 & 1 & 0 & 9 & 1 & 0 & 9 \\
\hline $20 \mathrm{~dB}$ & 1 & 0 & 9 & 1 & 0 & 9 & 0 & 0 & 10 \\
\hline
\end{tabular}




\section{A.2 PPV and sensitivity at varying SQI threshold levels for each SQVS configuration}

\begin{tabular}{|c|c|c|c|c|c|c|}
\hline \multirow{2}{*}{$\begin{array}{c}\text { SQI } \\
\text { Threshold }\end{array}$} & \multicolumn{2}{|c|}{$S Q V S_{S T}$} & \multicolumn{2}{|c|}{$S Q V S_{E N}$} & \multicolumn{2}{|c|}{$S Q V S_{A L}$} \\
\hline & PPV & Sensitivity & PPV & Sensitivity & PPV & Sensitivity \\
\hline$-15 \mathrm{~dB}$ & 0.39 & 0.90 & 0.39 & 0.90 & 0.39 & 0.90 \\
\hline$-14 \mathrm{~dB}$ & 0.39 & 0.90 & 0.39 & 0.90 & 0.39 & 0.90 \\
\hline$-13 \mathrm{~dB}$ & 0.39 & 0.90 & 0.39 & 0.90 & 0.41 & 0.90 \\
\hline$-12 \mathrm{~dB}$ & 0.39 & 0.90 & 0.39 & 0.90 & 0.41 & 0.90 \\
\hline$-11 \mathrm{~dB}$ & 0.39 & 0.90 & 0.39 & 0.90 & 0.41 & 0.90 \\
\hline$-10 \mathrm{~dB}$ & 0.38 & 0.90 & 0.39 & 0.90 & 0.43 & 0.90 \\
\hline$-9 \mathrm{~dB}$ & 0.39 & 0.90 & 0.45 & 0.90 & 0.53 & 0.90 \\
\hline$-8 \mathrm{~dB}$ & 0.39 & 0.90 & 0.53 & 0.80 & 0.57 & 0.80 \\
\hline$-7 \mathrm{~dB}$ & 0.50 & 0.90 & 0.57 & 0.80 & 0.62 & 0.80 \\
\hline$-6 \mathrm{~dB}$ & 0.47 & 0.80 & 0.62 & 0.80 & 0.80 & 0.80 \\
\hline$-5 \mathrm{~dB}$ & 0.56 & 1.00 & 0.69 & 0.90 & 0.80 & 0.80 \\
\hline$-4 \mathrm{~dB}$ & 0.69 & 0.90 & 0.82 & 0.90 & 0.80 & 0.80 \\
\hline$-3 \mathrm{~dB}$ & 0.82 & 0.90 & 0.82 & 0.90 & 0.89 & 0.80 \\
\hline$-2 \mathrm{~dB}$ & 0.75 & 0.90 & 0.89 & 0.80 & 1.00 & 0.80 \\
\hline$-1 \mathrm{~dB}$ & 0.62 & 0.80 & 0.89 & 0.80 & 1.00 & 0.80 \\
\hline $0 \mathrm{~dB}$ & 0.80 & 0.80 & 1.00 & 0.80 & 1.00 & 0.80 \\
\hline $1 \mathrm{~dB}$ & 0.89 & 0.80 & 1.00 & 0.80 & 1.00 & 0.80 \\
\hline $2 \mathrm{~dB}$ & 0.80 & 0.80 & 1.00 & 0.80 & 1.00 & 0.80 \\
\hline $3 \mathrm{~dB}$ & 0.80 & 0.80 & 1.00 & 0.80 & 1.00 & 0.80 \\
\hline $4 \mathrm{~dB}$ & 0.80 & 0.80 & 1.00 & 0.80 & 1.00 & 0.80 \\
\hline $5 \mathrm{~dB}$ & 0.89 & 0.80 & 1.00 & 0.80 & 1.00 & 0.80 \\
\hline $6 \mathrm{~dB}$ & 0.89 & 0.80 & 1.00 & 0.80 & 1.00 & 0.80 \\
\hline $7 \mathrm{~dB}$ & 0.89 & 0.80 & 1.00 & 0.80 & 1.00 & 0.80 \\
\hline $8 \mathrm{~dB}$ & 0.89 & 0.80 & 1.00 & 0.80 & 1.00 & 0.80 \\
\hline $9 \mathrm{~dB}$ & 0.89 & 0.80 & 1.00 & 0.80 & 1.00 & 0.80 \\
\hline $10 \mathrm{~dB}$ & 0.89 & 0.80 & 1.00 & 0.80 & 1.00 & 0.80 \\
\hline $11 \mathrm{~dB}$ & 1.00 & 0.80 & 1.00 & 0.80 & 1.00 & 0.80 \\
\hline $12 \mathrm{~dB}$ & 1.00 & 0.80 & 1.00 & 0.80 & 1.00 & 0.80 \\
\hline $13 \mathrm{~dB}$ & 1.00 & 0.80 & 1.00 & 0.80 & 1.00 & 0.80 \\
\hline $14 \mathrm{~dB}$ & 1.00 & 0.80 & 1.00 & 0.70 & 1.00 & 0.70 \\
\hline $15 \mathrm{~dB}$ & 1.00 & 0.80 & 1.00 & 0.70 & 1.00 & 0.70 \\
\hline $16 \mathrm{~dB}$ & 0.89 & 0.80 & 1.00 & 0.70 & 1.00 & 0.60 \\
\hline $17 \mathrm{~dB}$ & 1.00 & 0.70 & 1.00 & 0.60 & 1.00 & 0.30 \\
\hline $18 \mathrm{~dB}$ & 1.00 & 0.60 & 1.00 & 0.20 & 1.00 & 0.20 \\
\hline $19 \mathrm{~dB}$ & 1.00 & 0.10 & 1.00 & 0.10 & 1.00 & 0.10 \\
\hline $20 \mathrm{~dB}$ & 1.00 & 0.10 & 1.00 & 0.10 & $\mathrm{NaN}^{1}$ & 0 \\
\hline
\end{tabular}

\footnotetext{
${ }^{1}$ In this case the PPV cannot be calculated because of the absence of both TP and FP. Equation (6.1) results
} in division by 0 , making the calculation invalid. 


\section{Appendix B: Statistical Counts - Second Run}

B.1 TP, FP, and FN counts for alarms modified at varying SQI threshold levels by each SQVS configuration

\begin{tabular}{|c|c|c|c|c|c|c|c|c|c|}
\hline \multirow{2}{*}{$\begin{array}{c}\text { SQI } \\
\text { Threshold }\end{array}$} & \multicolumn{3}{|c|}{$S Q V S_{S T}$} & \multicolumn{3}{|c|}{$S Q V S_{E N}$} & \multicolumn{3}{|c|}{$\boldsymbol{S Q V} \boldsymbol{S}_{A L}$} \\
\hline & TP & FP & FN & TP & FP & FN & TP & FP & FN \\
\hline$-15 \mathrm{~dB}$ & 9 & 12 & 1 & 9 & 12 & 1 & 9 & 12 & 1 \\
\hline$-14 \mathrm{~dB}$ & 9 & 12 & 1 & 9 & 12 & 1 & 9 & 12 & 1 \\
\hline$-13 \mathrm{~dB}$ & 9 & 12 & 1 & 9 & 12 & 1 & 9 & 12 & 1 \\
\hline$-12 \mathrm{~dB}$ & 9 & 13 & 1 & 9 & 12 & 1 & 9 & 12 & 1 \\
\hline$-11 \mathrm{~dB}$ & 9 & 14 & 1 & 9 & 13 & 1 & 9 & 11 & 1 \\
\hline$-10 \mathrm{~dB}$ & 9 & 12 & 1 & 9 & 11 & 1 & 9 & 7 & 1 \\
\hline$-9 \mathrm{~dB}$ & 9 & 13 & 1 & 9 & 7 & 1 & 9 & 7 & 1 \\
\hline$-8 \mathrm{~dB}$ & 9 & 11 & 1 & 9 & 6 & 1 & 9 & 6 & 1 \\
\hline$-7 \mathrm{~dB}$ & 9 & 11 & 1 & 9 & 6 & 1 & 9 & 5 & 1 \\
\hline$-6 \mathrm{~dB}$ & 9 & 7 & 1 & 9 & 3 & 1 & 9 & 3 & 1 \\
\hline$-5 \mathrm{~dB}$ & 9 & 6 & 1 & 9 & 2 & 1 & 9 & 2 & 1 \\
\hline$-4 \mathrm{~dB}$ & 9 & 5 & 1 & 9 & 2 & 1 & 9 & 2 & 1 \\
\hline$-3 \mathrm{~dB}$ & 9 & 4 & 1 & 9 & 2 & 1 & 9 & 2 & 1 \\
\hline$-2 \mathrm{~dB}$ & 9 & 3 & 1 & 9 & 1 & 1 & 9 & 2 & 1 \\
\hline$-1 \mathrm{~dB}$ & 9 & 6 & 1 & 9 & 1 & 1 & 9 & 2 & 1 \\
\hline $0 \mathrm{~dB}$ & 9 & 2 & 1 & 9 & 0 & 1 & 9 & 1 & 1 \\
\hline $1 \mathrm{~dB}$ & 9 & 0 & 1 & 9 & 0 & 1 & 9 & 1 & 1 \\
\hline $2 \mathrm{~dB}$ & 9 & 0 & 1 & 9 & 0 & 1 & 9 & 1 & 1 \\
\hline $3 \mathrm{~dB}$ & 9 & 1 & 1 & 9 & 0 & 1 & 9 & 1 & 1 \\
\hline $4 \mathrm{~dB}$ & 9 & 1 & 1 & 9 & 0 & 1 & 9 & 1 & 1 \\
\hline $5 \mathrm{~dB}$ & 9 & 0 & 1 & 9 & 0 & 1 & 9 & 1 & 1 \\
\hline $6 \mathrm{~dB}$ & 9 & 0 & 1 & 9 & 0 & 1 & 9 & 1 & 1 \\
\hline $7 \mathrm{~dB}$ & 9 & 0 & 1 & 9 & 0 & 1 & 9 & 1 & 1 \\
\hline $8 \mathrm{~dB}$ & 9 & 0 & 1 & 9 & 0 & 1 & 9 & 1 & 1 \\
\hline $9 \mathrm{~dB}$ & 9 & 0 & 1 & 9 & 0 & 1 & 9 & 1 & 1 \\
\hline $10 \mathrm{~dB}$ & 9 & 0 & 1 & 9 & 0 & 1 & 9 & 1 & 1 \\
\hline $11 \mathrm{~dB}$ & 9 & 0 & 1 & 9 & 0 & 1 & 9 & 1 & 1 \\
\hline $12 \mathrm{~dB}$ & 9 & 0 & 1 & 9 & 0 & 1 & 9 & 1 & 1 \\
\hline $13 \mathrm{~dB}$ & 9 & 0 & 1 & 9 & 0 & 1 & 9 & 1 & 1 \\
\hline $14 \mathrm{~dB}$ & 9 & 0 & 1 & 8 & 0 & 2 & 8 & 1 & 2 \\
\hline $15 \mathrm{~dB}$ & 8 & 0 & 2 & 8 & 0 & 2 & 8 & 1 & 2 \\
\hline $16 \mathrm{~dB}$ & 8 & 0 & 2 & 7 & 0 & 3 & 6 & 1 & 4 \\
\hline $17 \mathrm{~dB}$ & 8 & 0 & 2 & 6 & 0 & 4 & 3 & 1 & 7 \\
\hline $18 \mathrm{~dB}$ & 6 & 0 & 4 & 2 & 0 & 8 & 2 & 0 & 8 \\
\hline $19 \mathrm{~dB}$ & 2 & 0 & 8 & 1 & 0 & 9 & 1 & 0 & 9 \\
\hline $20 \mathrm{~dB}$ & 1 & 0 & 9 & 1 & 0 & 9 & 0 & 0 & 10 \\
\hline
\end{tabular}




\section{B.2 PPV and sensitivity at varying SQI threshold levels for each SQVS configuration}

\begin{tabular}{|c|c|c||c|c||c|c|}
\hline \multirow{2}{*}{$\begin{array}{c}\text { SQI } \\
\text { Threshold }\end{array}$} & \multicolumn{2}{|c||}{$\boldsymbol{S Q V} \boldsymbol{V}_{\boldsymbol{S T}}$} & \multicolumn{2}{c||}{$\boldsymbol{S Q V} \boldsymbol{S}_{\boldsymbol{E N}}$} & \multicolumn{2}{c|}{$\boldsymbol{S Q V \boldsymbol { S } _ { \boldsymbol { A L } }}$} \\
\hline & PPV & Sensitivity & PPV & Sensitivity & PPV & Sensitivity \\
\hline$-15 \mathrm{~dB}$ & 0.43 & 0.90 & 0.43 & 0.90 & 0.43 & 0.90 \\
\hline$-14 \mathrm{~dB}$ & 0.43 & 0.90 & 0.43 & 0.90 & 0.43 & 0.90 \\
\hline$-13 \mathrm{~dB}$ & 0.43 & 0.90 & 0.43 & 0.90 & 0.43 & 0.90 \\
\hline$-12 \mathrm{~dB}$ & 0.41 & 0.90 & 0.43 & 0.90 & 0.43 & 0.90 \\
\hline$-11 \mathrm{~dB}$ & 0.39 & 0.90 & 0.41 & 0.90 & 0.45 & 0.90 \\
\hline$-10 \mathrm{~dB}$ & 0.43 & 0.90 & 0.45 & 0.90 & 0.56 & 0.90 \\
\hline$-9 \mathrm{~dB}$ & 0.41 & 0.90 & 0.56 & 0.90 & 0.56 & 0.90 \\
\hline$-8 \mathrm{~dB}$ & 0.45 & 0.90 & 0.60 & 0.90 & 0.60 & 0.90 \\
\hline$-7 \mathrm{~dB}$ & 0.45 & 0.90 & 0.60 & 0.90 & 0.64 & 0.90 \\
\hline$-6 \mathrm{~dB}$ & 0.56 & 0.90 & 0.75 & 0.90 & 0.75 & 0.90 \\
\hline$-5 \mathrm{~dB}$ & 0.60 & 0.90 & 0.82 & 0.90 & 0.82 & 0.90 \\
\hline$-4 \mathrm{~dB}$ & 0.64 & 0.90 & 0.82 & 0.90 & 0.82 & 0.90 \\
\hline$-3 \mathrm{~dB}$ & 0.69 & 0.90 & 0.82 & 0.90 & 0.82 & 0.90 \\
\hline$-2 \mathrm{~dB}$ & 0.75 & 0.90 & 0.90 & 0.90 & 0.82 & 0.90 \\
\hline$-1 \mathrm{~dB}$ & 0.60 & 0.90 & 0.90 & 0.90 & 0.82 & 0.90 \\
\hline $0 \mathrm{~dB}$ & 0.82 & 0.90 & 1.00 & 0.90 & 0.90 & 0.90 \\
\hline $1 \mathrm{~dB}$ & 1.00 & 0.90 & 1.00 & 0.90 & 0.90 & 0.90 \\
\hline $2 \mathrm{~dB}$ & 1.00 & 0.90 & 1.00 & 0.90 & 0.90 & 0.90 \\
\hline $3 \mathrm{~dB}$ & 0.90 & 0.90 & 1.00 & 0.90 & 0.90 & 0.90 \\
\hline $4 \mathrm{~dB}$ & 0.90 & 0.90 & 1.00 & 0.90 & 0.90 & 0.90 \\
\hline $5 \mathrm{~dB}$ & 1.00 & 0.90 & 1.00 & 0.90 & 0.90 & 0.90 \\
\hline $6 \mathrm{~dB}$ & 1.00 & 0.90 & 1.00 & 0.90 & 0.90 & 0.90 \\
\hline $7 \mathrm{~dB}$ & 1.00 & 0.90 & 1.00 & 0.90 & 0.90 & 0.90 \\
\hline $8 \mathrm{~dB}$ & 1.00 & 0.90 & 1.00 & 0.90 & 0.90 & 0.90 \\
\hline $9 \mathrm{~dB}$ & 1.00 & 0.90 & 1.00 & 0.90 & 0.90 & 0.90 \\
\hline $10 \mathrm{~dB}$ & 1.00 & 0.90 & 1.00 & 0.90 & 0.90 & 0.90 \\
\hline $11 \mathrm{~dB}$ & 1.00 & 0.90 & 1.00 & 0.90 & 0.90 & 0.90 \\
\hline $12 \mathrm{~dB}$ & 1.00 & 0.90 & 1.00 & 0.90 & 0.90 & 0.90 \\
\hline $13 \mathrm{~dB}$ & 1.00 & 0.90 & 1.00 & 0.90 & 0.90 & 0.90 \\
\hline $14 \mathrm{~dB}$ & 1.00 & 0.90 & 1.00 & 0.80 & 0.89 & 0.80 \\
\hline $15 \mathrm{~dB}$ & 1.00 & 0.80 & 1.00 & 0.80 & 0.89 & 0.80 \\
\hline $16 \mathrm{~dB}$ & 1.00 & 0.80 & 1.00 & 0.70 & 0.86 & 0.60 \\
\hline $17 \mathrm{~dB}$ & 1.00 & 0.80 & 1.00 & 0.60 & 0.75 & 0.30 \\
\hline $18 \mathrm{~dB}$ & 1.00 & 0.60 & 1.00 & 0.20 & 1.00 & 0.20 \\
\hline $19 \mathrm{~dB}$ & 1.00 & 0.20 & 1.00 & 0.10 & 1.00 & 0.10 \\
\hline $20 \mathrm{~dB}$ & 1.00 & 0.10 & 1.00 & 0.10 & NaN & 0.00 \\
\hline & & & & & & \\
\hline
\end{tabular}

${ }^{2}$ In this case the PPV cannot be calculated because of the absence of both TP and FP. Equation (6.1) results in division by 0 , making the calculation invalid. 


\section{Appendix C: PPV and Sensitivity for Averaged Counts}

\section{C.1 PPV and sensitivity at varying SQI threshold levels for each SQVS configuration}

\begin{tabular}{|c|c|c||c|c||c|c|}
\hline \multirow{2}{*}{$\begin{array}{c}\text { SQI } \\
\text { Threshold }\end{array}$} & \multicolumn{2}{|c||}{$\boldsymbol{S Q V} \boldsymbol{V}_{\boldsymbol{S T}}$} & \multicolumn{2}{c||}{$\boldsymbol{S Q V} \boldsymbol{S}_{\boldsymbol{E}}$} & \multicolumn{2}{c|}{$\boldsymbol{S Q V} \boldsymbol{S}_{\boldsymbol{A L}}$} \\
\hline & PPV & \multicolumn{1}{|c|}{ Sensitivity } & PPV & Sensitivity & PPV & Sensitivity \\
\hline$-15 \mathrm{~dB}$ & 0.41 & 0.90 & 0.41 & 0.90 & 0.41 & 0.90 \\
\hline$-14 \mathrm{~dB}$ & 0.41 & 0.90 & 0.41 & 0.90 & 0.41 & 0.90 \\
\hline$-13 \mathrm{~dB}$ & 0.41 & 0.90 & 0.41 & 0.90 & 0.42 & 0.90 \\
\hline$-12 \mathrm{~dB}$ & 0.40 & 0.90 & 0.41 & 0.90 & 0.42 & 0.90 \\
\hline$-11 \mathrm{~dB}$ & 0.39 & 0.90 & 0.40 & 0.90 & 0.43 & 0.90 \\
\hline$-10 \mathrm{~dB}$ & 0.40 & 0.90 & 0.42 & 0.90 & 0.49 & 0.90 \\
\hline$-9 \mathrm{~dB}$ & 0.40 & 0.90 & 0.50 & 0.90 & 0.55 & 0.90 \\
\hline$-8 \mathrm{~dB}$ & 0.42 & 0.90 & 0.57 & 0.85 & 0.57 & 0.85 \\
\hline$-7 \mathrm{~dB}$ & 0.47 & 0.90 & 0.59 & 0.85 & 0.61 & 0.85 \\
\hline$-6 \mathrm{~dB}$ & 0.52 & 0.85 & 0.68 & 0.85 & 0.68 & 0.85 \\
\hline$-5 \mathrm{~dB}$ & 0.58 & 0.95 & 0.75 & 0.90 & 0.81 & 0.85 \\
\hline$-4 \mathrm{~dB}$ & 0.67 & 0.90 & 0.82 & 0.90 & 0.81 & 0.85 \\
\hline$-3 \mathrm{~dB}$ & 0.75 & 0.90 & 0.82 & 0.90 & 0.81 & 0.85 \\
\hline$-2 \mathrm{~dB}$ & 0.75 & 0.90 & 0.89 & 0.85 & 0.85 & 0.85 \\
\hline$-1 \mathrm{~dB}$ & 0.61 & 0.85 & 0.89 & 0.85 & 0.89 & 0.85 \\
\hline $0 \mathrm{~dB}$ & 0.81 & 0.85 & 1.00 & 0.85 & 0.94 & 0.85 \\
\hline $1 \mathrm{~dB}$ & 0.94 & 0.85 & 1.00 & 0.85 & 0.94 & 0.85 \\
\hline $2 \mathrm{~dB}$ & 0.89 & 0.85 & 1.00 & 0.85 & 0.94 & 0.85 \\
\hline $3 \mathrm{~dB}$ & 0.85 & 0.85 & 1.00 & 0.85 & 0.94 & 0.85 \\
\hline $4 \mathrm{~dB}$ & 0.85 & 0.85 & 1.00 & 0.85 & 0.94 & 0.85 \\
\hline $5 \mathrm{~dB}$ & 0.94 & 0.85 & 1.00 & 0.85 & 0.94 & 0.85 \\
\hline $6 \mathrm{~dB}$ & 0.94 & 0.85 & 1.00 & 0.85 & 0.94 & 0.85 \\
\hline $7 \mathrm{~dB}$ & 0.94 & 0.85 & 1.00 & 0.85 & 0.94 & 0.85 \\
\hline $8 \mathrm{~dB}$ & 0.94 & 0.85 & 1.00 & 0.85 & 0.94 & 0.85 \\
\hline $9 \mathrm{~dB}$ & 0.94 & 0.85 & 1.00 & 0.85 & 0.94 & 0.85 \\
\hline $10 \mathrm{~dB}$ & 0.94 & 0.85 & 1.00 & 0.85 & 0.94 & 0.85 \\
\hline $11 \mathrm{~dB}$ & 1.00 & 0.85 & 1.00 & 0.85 & 0.94 & 0.85 \\
\hline $12 \mathrm{~dB}$ & 1.00 & 0.85 & 1.00 & 0.85 & 0.94 & 0.85 \\
\hline $13 \mathrm{~dB}$ & 1.00 & 0.85 & 1.00 & 0.85 & 0.94 & 0.85 \\
\hline $14 \mathrm{~dB}$ & 1.00 & 0.85 & 1.00 & 0.75 & 0.94 & 0.75 \\
\hline $15 \mathrm{~dB}$ & 1.00 & 0.80 & 1.00 & 0.75 & 0.94 & 0.75 \\
\hline $16 \mathrm{~dB}$ & 0.94 & 0.80 & 1.00 & 0.70 & 0.92 & 0.60 \\
\hline $17 \mathrm{~dB}$ & 1.00 & 0.75 & 1.00 & 0.60 & 0.86 & 0.30 \\
\hline $18 \mathrm{~dB}$ & 1.00 & 0.60 & 1.00 & 0.20 & 1.00 & 0.20 \\
\hline $19 \mathrm{~dB}$ & 1.00 & 0.15 & 1.00 & 0.10 & 1.00 & 0.10 \\
\hline $20 \mathrm{~dB}$ & 1.00 & 0.10 & 1.00 & 0.10 & NaN & 0.00 \\
\hline & & & & & & \\
\hline
\end{tabular}

\footnotetext{
${ }^{3}$ In this case the PPV cannot be calculated because of the absence of both TP and FP. Equation (6.1) results in division by 0 , making the calculation invalid.
} 